\title{
Characterization of the Biochemical Properties of Nudt8, a Novel CoA-degrading Enzyme that Localizes to the Mitochondria
}

\author{
Evan W. Kerr \\ West Virginia University
}

Follow this and additional works at: https://researchrepository.wvu.edu/etd

Part of the Biochemistry Commons

\section{Recommended Citation \\ Kerr, Evan W., "Characterization of the Biochemical Properties of Nudt8, a Novel CoA-degrading Enzyme that Localizes to the Mitochondria" (2020). Graduate Theses, Dissertations, and Problem Reports. 7831. https://researchrepository.wvu.edu/etd/7831}

This Dissertation is protected by copyright and/or related rights. It has been brought to you by the The Research Repository @ WVU with permission from the rights-holder(s). You are free to use this Dissertation in any way that is permitted by the copyright and related rights legislation that applies to your use. For other uses you must obtain permission from the rights-holder(s) directly, unless additional rights are indicated by a Creative Commons license in the record and/ or on the work itself. This Dissertation has been accepted for inclusion in WVU Graduate Theses, Dissertations, and Problem Reports collection by an authorized administrator of The Research Repository @ WVU. For more information, please contact researchrepository@mail.wvu.edu. 


\title{
Characterization of the Biochemical Properties of Nudt8, a Novel CoA-degrading
}

\author{
Evan William Kerr \\ Dissertation submitted \\ to the School of Medicine \\ at West Virginia University
}

Enzyme that Localizes to the Mitochondria

in partial fulfillment of the requirements for the degree of

Doctor of Philosophy in

Biochemistry and Molecular Biology

\author{
Roberta Leonardi, Ph.D., Chair \\ David Smith, Ph.D. \\ Bradley Webb, Ph.D. \\ Jianhai Du, Ph.D. \\ John Hollander, Ph.D
}

Department of Biochemistry and Molecular Biology

Morgantown, West Virginia

2020

Keywords: Coenzyme A, Nudix hydrolase, liver metabolism, fatty acid oxidation, mitochondria 


\section{Abstract \\ Characterization of the Biochemical Properties of Nudt8, a Novel CoA-degrading Enzyme that Localizes to the Mitochondria}

\section{Evan Kerr}

Coenzyme $\mathrm{A}(\mathrm{CoA})$ is a vital cofactor that is required for a variety of metabolic reactions including the TCA cycle and the synthesis and oxidation of fatty acids, amino acids and ketone bodies. The importance of $\mathrm{CoA}$ is underscored by its tight regulation, as prolonged elevations or inability to synthesize adequate amounts of this cofactor lead to severe metabolic dysfunction. Regulation of CoA biosynthesis has been extensively characterized, however less is known about regulation of $\mathrm{CoA}$ and its thioesters via degradation. Presently, two CoA-degrading enzymes, Nudt7 and Nudt19 have been identified as regulators of the peroxisomal pool of (acyl-)CoA in the liver and kidneys, respectively. In addition to the peroxisomes, pools of CoA and (acyl-)CoAs exist in the mitochondria and cytosol. Presently, no CoA-degrading enzymes localizing to these compartments have been identified. Nudt8 has been annotated as CoA-degrading enzyme due to its similarity to Nudt7. The CoA-binding motif and Nudix box are highly conserved between Nudt8 and Nudt7; however, the ability of Nudt8 to degrade (acyl-)CoAs had not been established experimentally. We were able to successfully express and purify recombinant Nudt8 and, using enzymatic assays, we confirmed that this enzyme is a CoA diphosphohydrolase specific for free CoA and medium chain acyl-CoAs. Furthermore, we were able to show that Nudt8 localizes to the mitochondrial matrix, positioning it to regulate the largest pool of (acyl-)CoAs in the cell. This research identified a novel CoA-degrading enzyme as a novel means through which mitochondrial CoA, and thus mitochondrial metabolism, is regulated. 


\section{Acknowledgments}

I would like to thank the Biochemistry department as a whole, and anyone who has helped me throughout this process. I would especially like to thank my advisor, Dr. Leonardi for being my mentor. I would also like to thank my committee Dr. Smith, Dr. Du, Dr. Hollander, and Dr. Webb for their input and constructive criticism. I would also like to thank the members of the "RNA and Metabolism" group that always gave valuable and constructive criticism on my work. Finally, I would like to thank all my lab-mates for their help throughout the years. I would especially like to thank Debbie and Steph for being great teachers and coworkers.

I would also like to thank my family. I have an awesome and supportive family and I

couldn't have done this without you guys. And to friends as well, thanks for always being there for me. 


\section{Abbreviations}

$\begin{array}{ll}\text { AAV } & \text { Adeno-associated virus } \\ \text { ACOT } & \text { Acyl-CoA thioesterase } \\ \text { APEX } & \text { Ascorbate Peroxidase } \\ \text { AT-1 } & \text { Acetyl-CoA transporter 1 } \\ \text { CBP } & \text { CREB-binding protein } \\ \text { CDCA } & \text { Chenodeoxycholic acid } \\ \text { CoA } & \text { Coenzyme A } \\ \text { COASY } & \text { CoA synthase } \\ \text { CROT } & \text { Carnitine octanolytransferase } \\ \text { DP-CoA } & \text { Dephospho-CoA } \\ \text { ER } & \text { Endoplasmic reticulum } \\ \text { GAPDH } & \text { Glyceraldehyde-3-phosphate dehydrogenase } \\ \text { mBB } & \text { Monobromobimane } \\ \text { MTS } & \text { Mitochondrial targeting sequence } \\ \text { NBIA } & \text { Neurodegeneration with brain iron accumulation } \\ \text { PANK } & \text { Pantothenate kinase } \\ \text { PKAN } & \text { Pantothenate Kinase-Associated Neurodegeneration } \\ \text { PI3K } & \text { Phosphoinositide 3- Kinase } \\ \text { PPARa } & \text { Peroxisome proliferator-activated receptor a } \\ \text { PTS } & \text { Peroxisomal targeting sequence } \\ \text { SLC25 } & \text { Solute Carrier Family 25 }\end{array}$




\section{Table of Contents}

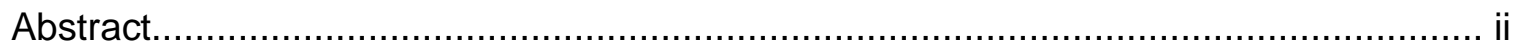

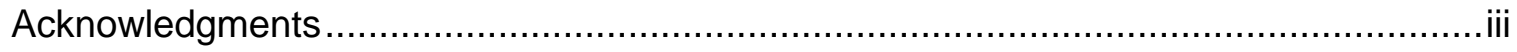

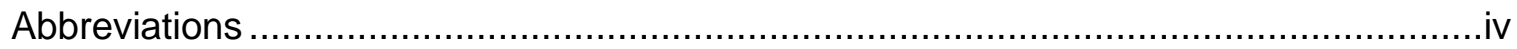

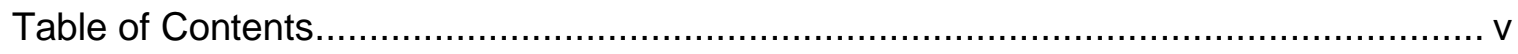

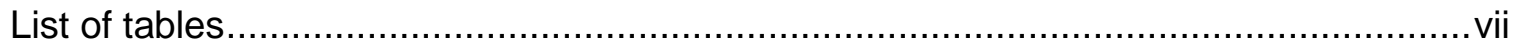

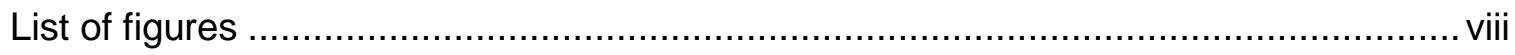

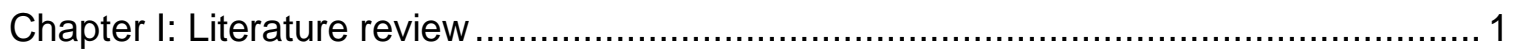

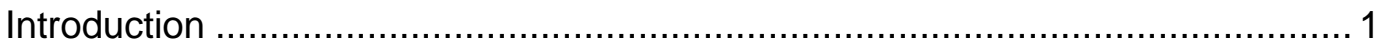

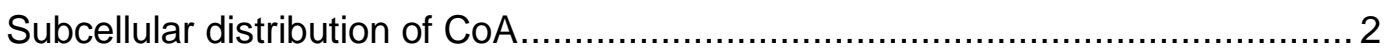

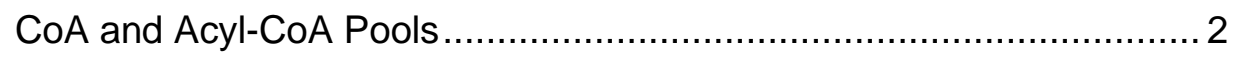

Mitochondrial CoA Transporters........................................................ 3

Peroxisomal CoA Transporters ......................................................... 5

Endoplasmic Reticulum CoA Transporter ........................................... 5

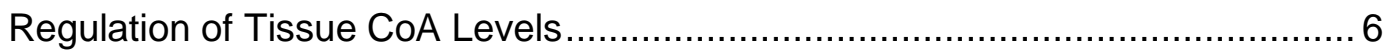

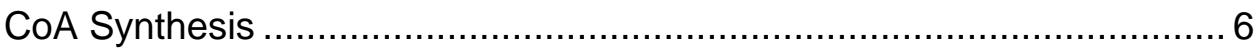

Metabolic Consequences of Supraphysiological CoA Levels ................... 9

Metabolic Consequences of Impaired CoA Synthesis ............................ 9

Regulation of CoA Through Degradation ..................................................... 10

Nudix Hydrolase Superfamily ........................................................... 11

Murine and Human CoA Degrading Enzymes....................................... 12

Acyl-CoA Thioesterase Mediated CoA Regulation ……......................... 13

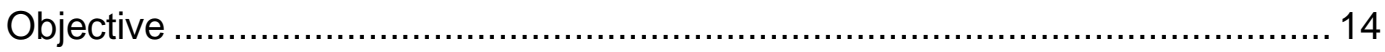

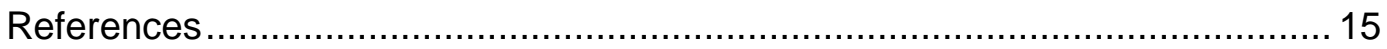


Chapter II: Nudt8 is a Novel CoA Diphosphohydrolase that Resides in the Mitochondria 20
Abstract
Introduction 21
Material and methods 23
Results 27
Discussion 34
References. 37

Chapter III: : Effect of liver specific overexpression of Nudt8 on mitochondrial metabolism

Abstract ............ 40

Introduction 41

Materials and methods 43

Results. 48

Discussion 56

References. 59

Chapter IV: Conclusions and future directions ... 62

Conclusions of chapter II .63

Future directions of chapter II. 62

Conclusions of chapter III . 66

Future directions of chapter III .66

Conclusions 68

References 70 


\section{List of tables}

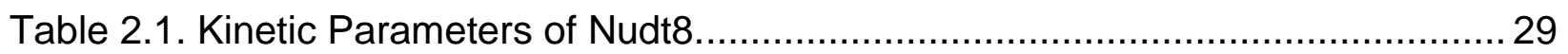




\section{List of figures}

Figure 1.1. Compartmentalization of CoA.

Figure 2.1. Expression and charactarization of mouse Nudt8 ............................... 27

Figure 2.2. Divalent cation requirment and substrate specificity of recombinant Nudt8 28

Figure 2.3. Relative abundance and tissue distribution of Nudt8 mRNA and protein. ... 30

Figure 2.4. Nudt8 expression in the liver, kidneys and heart of fed and fasted mice..... 31

Figure 2.5. Mitochondrial loclaiztation of Nudt8 ........................................................ 33

Figure 3.1. Nudt8 localizes to the mitochondrial matrix............................................ 49

Figure 3.2. APEX mediated biotinylation of Nudt8 confirms localization to mitochondrial

matrix

Figure 3.3.Liver specific over-expresion of Nudt8 increases CoA degrading activity in the mitochondria. 52

Figure 3.4. Blood Glucose and $\beta$-hydroxybutyrate were not affected by Nudt8

overexpression 53

Figure 3.5. Mitochondrial and cytosolic total CoA did not change in Nudt8-AAV mice.. 54

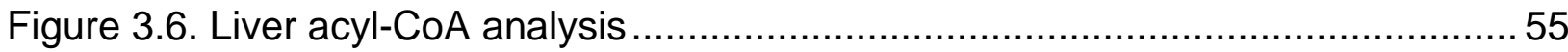

Figure 3.7. Compensation of Nudt8 mediated CoA degradation by Coasy................58 


\section{Chapter I: Literature review}

\section{Introduction}

Coenzyme $\mathrm{A}(\mathrm{CoA})$ is an essential cofactor that binds and activates organic acids within the cell for a multitude of reactions involved in energy metabolism (1). CoA is synthesized in its free, unacylated form from pantothenic acid (vitamin B5), ATP, and cysteine in a five-step pathway discussed in detail below (1). The reactive thiol of CoA forms thioester bonds with the carboxylic group of a multitude of compounds. Hydrolysis of the resulting thioester bond is energetically favorable $(-32-36 \mathrm{~kJ} / \mathrm{mol})$ and it is used to drive hundreds of reactions $(2,3)$. Within the cell, CoA and its thioesters are required for a variety of metabolic pathways including the tricarboxylic acid (TCA) cycle, fatty acid $\beta$-oxidation, lipogenesis, acetylcholine synthesis, as well as ketone body synthesis and oxidation. Additionally, through acylation of proteins, CoA thioesters can regulate protein function, protein localization, stability, and protein-protein interactions $(4,5)$. Under conditions of oxidative stress, the thiol of free CoA can directly react with cysteines of target proteins, which is referred to as 'CoAlation' (6). Free CoA and acyl-CoAs can also regulate metabolic pathways through allosteric regulation of key enzymes interactions with target proteins, allowing for fine tuning of metabolic activities within the cell $(7,8)$.

At the whole tissue level, total CoA levels (free CoA and acyl-CoAs) dynamically change with the nutritional state. In the liver and kidneys, the fasted state is associated with increased CoA synthesis that promotes $\beta$-oxidation, ketogenesis, and gluconeogenesis (9). Conversely, the switch from fatty acid to glucose oxidation is promoted, in the fed state, through a net decrease in free and acyl-CoAs (10). The net decrease in CoA occurs from both inhibition of CoA synthesis and increasing CoA degradation (10). Through coordination of synthesis and degradation, total CoA levels are tightly regulated, which allows the cell to adapt to different metabolic states. The importance of CoA homeostasis is underscored by the fact that prolonged elevations of total CoA 
levels contribute to excess hepatic fatty acid oxidation and gluconeogenesis seen in Type 2 Diabetes (11). Conversely, genetic or chemical inhibition of CoA synthesis results in a rapid decrease in tissue total CoA levels, inhibition of fatty acid oxidation, and severe fasting hypoglycemia $(9,12)$. Thus, tight regulation of CoA and acyl-CoA levels is essential for metabolic regulation of the cell.

\section{Subcellular Distribution of CoA}

\section{CoA and Acyl-CoA Pools}

CoA biosynthesis is ubiquitous and generate free CoA, which can then be used to activate organic acids as acylCoAs (Fig. 1.1). The concentration of total CoA changes with the metabolic needs of the cell/tissue/organ

$(13,14)$. Within the cell, $\mathrm{CoA}$ and its derivatives are found in three major subcellular pools in the

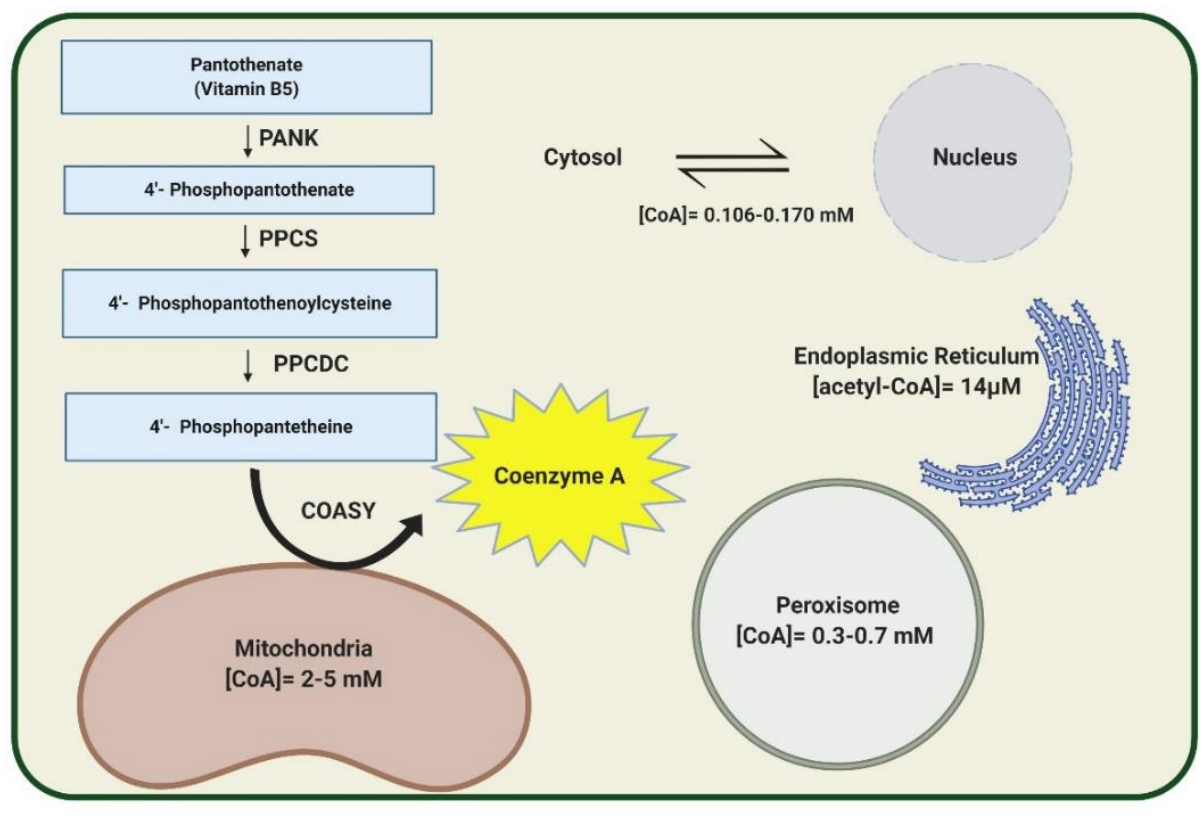

mitochondria, cytosol,

Figure 1.1: Compartmentalization of CoA. The initial steps of CoA synthesis take place in the cytosol. The final steps of CoA synthesis are catalyzed by

and peroxisomes. CoA synthase, which has been annotated to reside in both the mitochondrial matrix and outer membrane. Thus, it is unclear where the final two steps of

These pools support CoA synthesis take place. The mitochondrial CoA pool contains as much as $80 \%$ of the cellular $\mathrm{CoA}$ pool. The peroxisomal $\mathrm{CoA}$ pool is the second largest

processes that are pool of CoA in the cell supporting fatty acid oxidation and bile acid synthesis. Both the peroxisomes and the mitochondria contain CoA-degrading Nudix specific to each compartment. The mitochondria contain hydrolases that act to regulate $\mathrm{CoA}$ dependent reactions occurring within the organelle. The cytosol/ nuclear pool is the smallest pool of CoA used for lipogenesis and acetylation of proteins. Finally, the endoplasmic reticulum contains a small pool of acetyl CoA utilized for post translational modifications mediating protein stability. 
the largest pool of acyl-CoA. It is estimated that in the heart, the mitochondria contain as much as $95 \%$ of the total cellular CoA with the remaining $5 \%$ divided between the cytosol and peroxisomes (10). In the liver, $80 \%$ of the (acyl-)CoA pool is mitochondrial, with the remaining $20 \%$ allocated to the cytosol and peroxisomes (15-17). The mitochondrial CoA/acyl-CoA pool supports a variety of metabolic pathways including fatty acid $\beta$-oxidation, ketone synthesis, gluconeogenesis, and the TCA cycle. The cytosolic concentration of total CoA is estimated to be 0.106- $0.140 \mathrm{mM}$ support processes such as lipid synthesis $(15,16,18)$. Nuclear pores allow cytosolic (acyl-)CoAs to freely move between the cytosol and nucleus where histone aceyltransferases catalyze the acyl-CoA dependent transfer of acyl-groups to lysines on histones as a means to regulate gene expression (19). The peroxisomal (acyl-)CoA pool is utilized for the oxidation of very long chain fatty acids and the final steps in bile acid synthesis $(17,20)$. Finally, the endoplasmic reticulum (ER) contains a small pool of acetyl-CoA to act as acetyl-donor for acetyltransferases that modify ER proteins. Acetylation of ER proteins acts as a quality control mechanism regulating protein stability (21).

\section{Mitochondrial CoA Transporters:}

CoA species are unable to passively diffuse across lipid membranes, and the mitochondrial and peroxisomal CoA/acyl-CoA pools are formed through specific transporters. Acyl-CoAs entering the mitochondria for oxidization must first be converted to acyl-carnitines allowing them to be transported across the inner mitochondrial membrane via carnitine acylcarnitine translocase (22). Once in the mitochondrial matrix, the ester bond linking the carnitine and the acyl- group is cleaved, reforming the acyl-CoA via carnitine palmitoyl transferase 2 (22). Pools of free CoA in the mitochondria and peroxisomes are formed via transporters belonging to the Solute Carrier Family 25 (SLC25) superfamily (23). To date, two mitochondrial transporters involved in the transport of CoA species have been identified, SLC25A42 and SLC25A16 $(24,25)$. In humans, mutations of SLC25A42 results in a wide range of symptoms ranging from muscle 
weakness and lactic acidosis to developmental delay, encephalopathy, and epilepsy (26-28). In vitro reconstitution and analysis of recombinant SLC25A42 indicated that it catalyzes a counter exchange of free CoA $\left(K_{m}=71 \pm 10 \mu \mathrm{M}\right)$ or dephospho-CoA $\left(K_{m}=64 \pm 9 \mu \mathrm{M}\right)$ for 3', $5^{\prime}-A D P\left(K_{m}=\right.$ $55 \pm 8 \mu \mathrm{M})(24)$. Deletion of Leu5P, a mitochondrial CoA transporter in yeast, resulted in a 15fold decrease in total CoA in the mitochondria resulting in the inability to utilize fatty acids as substrates (25). The inability to utilize fatty acids was rescued with expression of the human transporter SLC25A42 (24). These results support the conclusion that Leu5P and SLC25A42 function to import free CoA into the mitochondrial matrix (24). Less is known about SLC25A16, a second mitochondrial free CoA transporter. In vitro expression and reconstitution of SLC25A16 has not been successful. However, its ability to transport free CoA into the mitochondria was also shown in the Leu5P deficient yeast model (25). Expression of SCL25A16 was able to partially rescue the CoA-deficient phenotype by increasing the mitochondrial total CoA (25). The cytosolic acyl-CoA/CoA pool was unaffected, suggesting that the effect of SCL25A16 maybe specific to the mitochondrial (acyl-)CoA pool.

The first three steps of CoA synthesis are catalyzed by enzymes in the cytosol. The final two steps of CoA synthesis are catalyzed by CoA synthase (Coasy), a bifunctional enzyme that localizes to the mitochondria (29). It was previously shown that Coasy localizes to the outer membrane, with the active site exposed to the cytosol; however, more recently, Coasy has also been shown to localize to the mitochondrial matrix (29-31). The discovery of Coasy in the mitochondrial matrix raises the questions of where the primary site of CoA synthesis is and what the role of these mitochondrial transporters is in the distribution of newly synthesized CoA to the rest of the cell. If Coasy localized to the outer-mitochondrial membrane, the final CoA produced by Coasy would be released into the cytosol and mitochondrial transporters would function to import CoA into the mitochondria. This hypothesis is supported by the finding that SLC25A16 rescues mitochondria total CoA levels without effecting cytosolic levels. However, determining the 
submitochondrial localization of Coasy is essential to establish where the final two steps in CoA biosynthesis occurs, which will provide insight into whether these transporters function to import CoA into the mitochondria, or export de novo synthesized CoA into the cytosol.

\section{Peroxisomal CoA Transporters:}

Like the mitochondria, the peroxisomal pool of free CoA is imported from the cytosol via SLC25 transporters. The peroxisomes also have a family of ATP Binding Cassette (ABCD 1-3) transporters that function to import acyl-CoAs into the peroxisome. These ABCD proteins bind long chain acyl-CoAs including the thioesters of linear, branched-chain and dicarboxylic fatty acids and hydrolyze their thioester bond; the imported fatty acid is thought to be re-acylated once inside the peroxisome (32). While the ABCD1-3 transporters import acyl-CoAs into the peroxisomes, SLC25A17 is potentially a transporter for free CoA. In vitro reconstitution of SLC25A17 found that this transporter catalyzes a counter exchange reaction exchanging ADP for CoA $(19.6 \pm 3.8 \mu \mathrm{M})$, dephospho-CoA $(14.4 \pm 5.4 \mu \mathrm{M})$, FAD $(2.6 \pm 1.3 \mu \mathrm{M})$, FMN $(10.2 \pm 3.3 \mu \mathrm{M})$ and 3',5'-ADP $(0.78 \pm 0.1 \mathrm{mM})$ (33). Deletion of SLC25A17 in zebra fish resulted in impaired oxidation of long chain fatty acids indicating, that it functions to import CoA and other cofactors to drive peroxisomal CoA-dependent processes (34). One of the primary functions of peroxisomes is the initial oxidation of very long-chain fatty acids, which are then completely oxidized in the mitochondria. Following oxidation in the peroxisomes, short- and medium-chain acyl-CoAs are converted to the corresponding acyl-carnitine by carnitine acetyltransferase (short chain acylCoAs with 2-4 carbons), or carnitine octanoyltransferase (medium- chain acyl-CoAs with up to 10 carbons) for complete oxidation in the mitochondria $(35,36)$.

\section{Endoplasmic Reticulum CoA Transporters:}

In the ER, a small pool of acetyl-CoA is present to act as acetyl-donor for acetyltransferases modifying proteins within the ER. Acetylation of ER proteins acts as a quality control mechanism regulating protein stability (37). Acetyl-CoA is transported into the ER by the 
transporter AT-1 (38). Unlike other members of the SLC family of proteins, AT-1 is specific for acetyl-CoA (38). The levels of acetyl-CoA within the ER are tightly regulated. Drastic decreases in acetyl-CoA levels induce autophagy and reduce cell viability. Mutations of AT-1 in murine models that lead to its inability to import acetyl-CoA result in decreased ER protein acetylation (38). This, in turn, causes developmental deficits, muscle weakness, and increased immune response through T-cell activation (39). Conversely, overexpression of AT-1 increased acetylation within the ER causes a wide variety of phenotypes including shortened life span, muscle atrophy, and effects on neuronal development $(40,41)$.

\section{$\underline{\text { Regulation of Tissue CoA Levels }}$}

\section{CoA Synthesis}

CoA synthesis occurs through a five-step pathway involving pantothenate (vitamin B5), ATP, and cysteine (Fig. 1.1) (1). The initial and rate-limiting step of CoA synthesis is the phosphorylation of pantothenate to 4'-phosphopantothenate, which is catalyzed by pantothenate kinase (PanK). There are four PanK isoforms, each sharing a common catalytic domain; however, each isoform has a distinct $\mathrm{N}$-terminal regulatory domain. The PanK1 gene encodes isoforms that are formed through alternative splicing, creating PanK1 $\alpha$ and PanK $1 \beta$, respectively (42). The difference between the two isoforms arises from the presence of a 184 amino acid $\mathrm{N}$-terminal extension present in the PanK1a isoform, which confers sensitivity to feed-back inhibition by CoA $\left(\mathrm{IC}_{50}=80 \mu \mathrm{M}\right)$, acetyl-CoA $\left(\mathrm{IC}_{50}=5 \mu \mathrm{M}\right)$, malonyl-CoA $\left(\mathrm{IC}_{50}=16 \mu \mathrm{M}\right)$ and long-chain acyl-CoAs $(42,43)$. The activity of PanK1 $\beta$, which lacks this regulatory domain, is unaffected by free and long-chain acyl-CoAs, and this isoform is less sensitive to inhibition by acetyl- and malonyl-CoA ( $\mathrm{IC}_{50}=32 \mu \mathrm{M}$ and $140 \mu \mathrm{M}$, respectively), compared to PanK1 $\alpha$ (43). PanK1 isoforms are primarily expressed in the liver, kidneys, and heart (42).

PanK2 has been studied extensively, due to its role in neurodegeneration. In humans, the primary difference between PANK2 and other isoforms is the presence of an N-terminal 
mitochondrial targeting sequence that targets PANK2 to the inner-mitochondrial space $(44,45)$. The murine isoform lacks this targeting sequence and is cytosolic $(44,45)$. Both the human and murine isoforms are potently inhibited by acetyl-CoA ( $\mathrm{IC}_{50}=62.5 \mathrm{nM}$ and $125 \mathrm{nM}$, respectively), while being activated by palmitoyl-carnitine (44). Murine PanK2 is a major isoform in brain and testes, and deletion results in retinal degeneration, weight loss and azoospermia $(44,46)$. In humans, PANK2 is the major isoform in the brain. Expression of PANK was highest in the cerebellum, caudate nucleus and cerebral cortex (44). Mutation in human PANK2 are associated with Pantothenate Kinase-Associated Neurodegeneration (PKAN), which is the most common form of Neurodegeneration with Brain Iron Accumulation (NBIA) (47). While the link between PANK2 mutations and PKAN has been firmly established, the exact mechanism through which PANK2 causes this neurodegenerative phenotype is still being elucidated.

The final pantothenate kinase isoform, PanK3, is ubiquitously expressed (48). Interestingly, PanK3 is $79 \%$ identical to PanK1 $\beta$ (48). Despite the high degree of similarity between the two isoforms, PanK3 is 10 times more sensitive to acetyl-CoA inhibition when compared to PanK1 $\beta$ and is also potently inhibited by palmitoyl-CoA $\left(\mathrm{IC}_{50}=2 \mu \mathrm{M}\right)$ (48). Interestingly, increasing PanK3 activity in mouse models of PKAN, ameliorated the neurodegenerative phenotype, pointing to PanK3 as an important therapeutic target (49).

Following formation by PanK, 4'-phosphopantothenate is condensed with cysteine to form 4'-phosphopantothenoylcysteine, which is then decarboxylated to generate 4'phosphopantetheine (1). These reactions take place in the cytosol and are catalyzed by 4'phosphopantothenoylcysteine synthase (PPCS) and 4'-phosphopantothenoylcysteine decarboxylase (PPCDC), respectively (Figure 1). There is a single isoform of PPCS and PPCDC enzymes and both are ubiquitously expressed (50). The final two steps in CoA biosynthesis are catalyzed by CoA synthase (COASY), a bifunctional enzyme that consists of a 4'phosphopantetheine adenyltransferase (PPAT) and a dephospho-CoA kinase (DPCK) domain 
$(29,51)$. There are two known isoforms of COASY, annotated COASYa and COASY $\beta$ (52). COASYa is a $60 \mathrm{kDa}$ protein that is ubiquitously expressed. COASY $\beta$ contains a 29 amino acid extension on the $\mathrm{N}$-terminus due to alternative splicing $(29,51,52)$. COASY $\beta$ mRNA is detectable in the brain, kidneys, and heart; however, expression of the protein is only detectable in brain (52). Unlike any of the previous components of the CoA biosynthetic pathway, both isoforms of COASY localize to the mitochondria (29). COASY has been annotated to localize to both the outer membrane and matrix of the mitochondria. Thus, determining the submitochondrial localization of COASY will be essential for determining the location of CoA biosynthesis, and to allow for a more complete understanding of how the cellular CoA pools are formed $(30,31)$.

Localization of COASY to the outer mitochondrial membrane is consistent with its reported interaction with proteins known to localize to the cytosol. COASY has been shown to interact with enhancer of mRNA-decapping protein 4 (EDC4) (53). This interaction was shown in vitro to inhibit the dephosphoCoA kinase activity of Coasy (53). Interestingly, removal of serum from culture medium to simulate a condition of fasting and increased CoA biosynthesis, prevented this interaction. Overexpression of EDC4 resulted in a 40\% decrease in CoA (53). More recently, Coasy was found to be phosphorylated by ribosomal protein S6 kinase 1 (S6K1) $(54,55)$. While the exact site of phosphorylation and the effect of this modification of Coasy activity have not been directly identified, in vitro experiments suggest that this modification may inhibit the PPAT activity of the enzyme (56).

Beyond regulation of the terminal steps in CoA biosynthesis, phosphorylation of Coasy has been shown to promote the interaction with the p85a regulatory subunit of Phosphoinositide 3-Kinase (PI3K), promoting phosphorylation of AKT and mTOR $(57,58)$. COASY expression in colorectal cancer cell lines was found to be protective against radiation therapy through increased PI3K signaling. Stable knockdown of COASY with shRNA in these cell lines reduced PI3K activity and induced radiation sensitivity in cells lines that were previously radiation resistant (57). 
Reduction of COASY through knockdown has also been shown to result in hyperacetylation of cytosolic proteins through activation CREB-binding protein (CBP) (59). CBP interacts with the PPAT domain of COASY, resulting in its inactivation and ultimately stalling mitosis and causing multinucleation (59).

\section{Metabolic Consequences of Supraphysiological CoA Levels}

Prolonged accumulations of CoA species have been shown to underlie disease states. For example, Leptin- deficient $\left(\operatorname{Lep}^{-/}\right)$mice exhibit a $40 \%$ increase in total hepatic CoA levels (11). This accumulation of (acyl-) CoA is due to a $30 \%$ increase in total PanK activity, and a coinciding decrease in the expression of the CoA-degrading enzyme Nudt7 (11). This increase in total hepatic CoA levels increases the rate of mitochondrial fatty acid $\beta$-oxidation. Acetyl-CoA produced from fatty acid oxidation act as an allosteric activator of the gluconeogenic enzyme pyruvate carboxylase, resulting in increased flux through the gluconeogenic pathway, which contributes to hyperglycemia (11). Deletion of Pank1 in $L e p^{-/}$mice resulted in a decrease in total hepatic CoA, which, in turn, reduced mitochondrial $\beta$-oxidation, and ameliorated the hyperglycemic phenotype independent of insulin signaling (11). Likewise, increased CoA synthesis in skeletal muscle through overexpression of human PANK2 led to increased oxidative stress, which resulted in decreased complex I activity, ATP production, and exercise intolerance (60).

\section{Metabolic Consequences of Impaired CoA Synthesis}

The importance of CoA and its thioesters is underscored by the severe metabolic defects that result from impaired CoA synthesis. Reduced hepatic CoA synthesis resulting from deletion of Pank1, decreased the rate of mitochondrial fatty acid oxidation. Reduced NADH and acetylCoA, which are normally derived from fatty acid oxidation, decreases the flux through the gluconeogenic pathway and caused severe fasting hypoglycemia (9). The synthesis of $\beta$ hydroxybutyrate, which also depends on acetyl-CoA and NADH derived from fatty acid oxidation, was also impaired (9). Global inhibition of CoA synthesis through treatment with Hopan, a potent 
inhibitor of all PanK isoforms, results in the inability to oxidize fatty acids and severe fasting hypoglycemia (12). Taken together, these data indicate that increased flux through the CoA biosynthetic pathways is essential for the transition from the fed to fasted state. Inadequate CoA synthesis due to PANK2 mutations underlie the dystonia, dysphagia, loss of vision and reduced cognitive function that under lie PKAN (47). PKAN patients exhibit increased plasma lactate in both the fasting and fed conditions, indicating a defect in mitochondrial metabolism (61). Importantly, allosteric activation of PanK3 in a PKAN mouse model using a small molecule, increased total CoA levels and reduced the severity of the PKAN phenotype (49).

\section{Regulation of CoA through Degradation}

In the fasted state, elevation of total tissue CoA levels is driven by increased flux through the CoA biosynthetic pathway via allosteric activation of the PanK isoforms by long-chain acylcarnitines, metabolites whose concentration increases in the fasted state. Upon refeeding, feedback inhibition of PanK by CoA thioesters predominates, resulting in decreased flux through the biosynthetic pathway. Concomitantly, active CoA degradation rapidly reduces tissue CoA levels (12). At just 2-hours after refeeding, hepatic CoA levels decrease as much as $28 \%$ and by 4-hours after refeeding, the levels decrease by $40 \%$ (11). Mice treated with Hopan, a pantothenate analog that inhibits all PanK isoforms, exhibit an $80 \%$ decrease in hepatic CoA levels, which indicate rapid turnover of CoA in this organ (12). Taken together, reduction in CoA levels occurs, not only through decreased synthesis, but also by active degradation. Intracellular CoA-degrading enzymes have been identified in the mitochondria, peroxisomes, and lysosomes. Within the lysosomes, CoA is dephosphoylated to dephospho-CoA (dP-CoA) likely by the lysosomal acid phosphatase (62). The conversion of CoA to dP-CoA within the lysosomes would occur as a part of autophagy. However, the role of this process in the regulation of CoA levels has not been elucidated. The currently identified mitochondrial and peroxisomal CoA-degrading enzymes belong to the Nudix hydrolase superfamily. 


\section{Nudix Hydrolase Superfamily}

Nudix hydrolases are a superfamily of pyrophosphohydrolases that perform a variety of vital functions within the cell including hydrolysis of oxidized nucleotides, RNA de-capping, hydrolysis of NDP-sugar conjugates and coenzymes (NAD(H), NADP(H), NMN, and CoA) (63). Nudix enzymes are expressed throughout all domains of life including eukaryotes, prokaryotes, archaea, and viruses (63). A 23 amino acid 'Nudix motif', GX ${ }_{5} E_{7}$ REUXEEXGU (U refers to an aliphatic, hydrophobic amino acid) is common to all members (64). The Nudix motif binds the essential divalent cation, most commonly $\mathrm{Mg}^{2+}$ or $\mathrm{Mn}^{2+}$, which activates a water molecule to hydrolyze the phosphosphodiester bond in the substrate (64). Substrate specificity is determined by various binding motifs. To date, CoA-degrading Nudix hydrolases have been characterized from Deinococcus radiodurans, Escherichia coli, Saccharomyces cerevisiae, Caenorhabditis elegans, Arabidopsis thaliana, mice and humans (65-71). The structure of a CoA pyrophosphohydrolase from Deinococcus radiodurans has been solved with the protein bound to $\mathrm{Mg}^{2+}$, identifying the catalytic residues $\mathrm{REX}_{2} \mathrm{EE}$ (69). This motif is conserved throughout all known CoA-degrading Nudix hydrolases including human and murine CoA-degrading enzymes (69). While the crystal structure of any CoA diphosphohydrolase with CoA bound has yet to be solved, the UPF0035 motif (LLTXRX ${ }_{3} \mathrm{RX}_{3} \mathrm{GX}_{3} \mathrm{FPGG}$ ) is predicted to participate in CoA binding and is highly conserved amongst CoA diphosphohydrolases (69). PCD1 and Y87G2A.14 are peroxisomal CoA diphosphohydrolases characterized from Saccharomyces cerevisiae and Caenorhabditis elegans, respectively. In vitro studies indicate that the preferred substrate for both enzymes are CoA disulfides, and the enzymes likely function to remove these byproducts of oxidative stress from the peroxisomal CoA pool $(65,66)$. However, recent studies of murine Nudix hydrolases indicate they have a much broader and complex role regarding CoA regulation. 


\section{Murine and Human Nudix Hydrolases}

In humans and mice, 22 Nudix hydrolases have been identified, two of which have been found to be specific diphosphohydrolases that hydrolyze (acyl-)CoA into 3',5'-ADP and acyl-4'phosphopantetheine. Nudt7 and Nudt19 are both peroxisomal CoA-degrading enzymes that are expressed in the liver and kidneys, respectively $(67,72)$. Both proteins contain a classic PTS1 peroxisomal targeting sequence on the C-terminus $(67,72)$. Nudt7 is a highly promiscuous CoAdegrading enzyme that, in vitro, hydrolyzes free $\mathrm{CoA}$, as well as a variety of short- and mediumchain acyl-CoAs $(72,73)$. Nudt7 is regulated transcriptionally. In the fasted state, when CoA is elevated, Nudt7 expression is minimal. Conversely, in the fed state, Nudt7 mRNA and protein robustly increase, suggesting that this enzyme helps to mediate the decrease in total hepatic CoA that is seen in the fed state (20). Overexpression of Nudt7 using a liver specific adeno-associated virus indicates that Nudt7 functions to regulate peroxisomal CoA dependent metabolic processes. Expression of the Nudt7 transgene resulted in a reduced rate of peroxisomal fatty acid oxidation leading to an accumulation of long-chain acyl-carnitines and reduced hexanoyl-, butyryl-, adipoyl, and succinyl-CoA, all products of peroxisomal fatty acid oxidation (20). Interestingly, Nudt7 overexpression resulted in decreased synthesis of primary and secondary bile acids (20). While these results have not been verified using a loss-of-function approach, this data suggests that Nudt7 regulates the peroxisomal CoA pool and CoA dependent metabolic reactions within the peroxisomes.

Nudt19 is another peroxisomal CoA-degrading enzyme that is expressed in the kidneys (70). In vitro data suggests that Nudt19 degrades free CoA as well as short- and medium-chain acyl-CoAs (72). Unlike Nudt7, Nudt19 shows very little to no activity towards acetyl-CoA. Nudt19 is also substantially larger than Nudt7 (42 kD vs. $27 \mathrm{kD}$ ) due to a $45-49$ amino acid insertion in the Nudix box that likely functions to assist with folding and stability of Nudt19 as deletion of this insertion results in the protein becoming insoluble (72). While it is unclear what specific pathways 
are effected by Nudt19 activity, deletion of Nudt19 in mice resulted in a $20 \%$ increase in total CoA in the fed state, indicating that Nudt19 contributes to the regulation of CoA within the kidney, in vivo (72).

\section{Acyl-CoA Thioesterase (ACOT)-mediated CoA Regulation}

Beyond removal of the 3' phosphate group or hydrolysis of the phosphodiester bond in the cofactor, the acyl-CoA pool can also be regulated through hydrolysis of the thioester linkage and release of unesterified fatty acids, a reaction that is catalyzed by a family of enzymes called acyl-CoA thioesterases (ACOTs) (74). There are two classifications of ACOTs. Type I ACOTS belong to the $\alpha / \beta$ - hydrolase superfamily and contain a catalytic triad consisting of a serine, histidine, and aspartic acid that are conserved throughout the $\alpha / \beta$ - hydrolase superfamily (74). Type I ACOTS share a high degree of sequence similarity and are thought to have evolved through gene duplication. Type II ACOTS share a low degree of sequence similarity; however they are structurally related by containing a "hotdog" fold (74). The majority of ACOTs localize to the mitochondria (ACOT 2,7,9,13) or the peroxisomes (ACOT 3,4,5,8,12) (75). Each ACOT has a very specific substrate preference ranging from short-chain, dicarboxylic, medium-chain, and long-chain acyl-CoAs. Type I ACOTs are mostly regulated transcriptionally and are targets of PPARa. Expression is induced by fasting, high fat diet, and ketogenic diets (76). Regulation of Type II ACOTs is less clear. Recently it was shown that mitochondrial type II ACOTs (ACOT 7,9 and 13) were all strongly inhibited by free-CoA leading to the hypothesis that activity is regulated by the ratio of acyl-CoAs/ free CoA within the mitochondria (77). 


\section{Objective}

Coenzyme $\mathrm{A}(\mathrm{Co} A)$ is an essential cofactor for a variety of metabolic pathways, most notably, the TCA cycle, lipid biosynthesis, amino acid synthesis and degradation, as well as fatty acid $\beta$-oxidation. CoA levels are tightly regulated. Inability to synthesize adequate levels of CoA is associated with neurological and developmental deficits. Conversely, elevated CoA levels in the liver are seen in the diabetic state and contribute to the hyperglycemic phenotype. CoA levels are dynamic. They increase in the fasted state and decrease in the fed state. Changes in the concentration of CoA species are mediated through cycles of synthesis and degradation. While biosynthesis of CoA has been studied extensively, the means through which this cofactor is degraded has yet to be completely elucidated. Two peroxisomal Nudix hydrolases, Nudt7 and Nudt19, have been found to catalyze the hydrolysis of the pyrophosphate bond of (acyl-)CoA creating (acyl-)phosphopantetheine and 3',5'-ADP. In addition to the peroxisomes, CoA is present in the cytosol as well as the mitochondria, but evidence for additional CoA-degrading enzymes in these subcellular compartments is missing. Nudt8 is an uncharacterized Nudix hydrolase that has been annotated as a CoA-degrading enzyme due to sharing $36 \%$ sequence identity to Nudt7, mainly in the catalytic region. The primary objective of this study is to express and purify recombinant Nudt8 and determine weather that Nudt8 is a CoA diphosphohydrolase. Nudt8 will be screened in vitro with a variety of short- and long-chain acyl-CoAs. Additionally, using a combination of in vitro and in vivo techniques, we will determine the mechanism regulating Nudt8 expression and activity. Finally, to determine in vivo CoA substrates, Nudt8 will be overexpressed in the liver of C57BL/6J mice to determine the effect of Nudt8 expression on the composition of the acyl-CoA pool in the liver. Untargeted metabolomics will be conducted on tissue samples from these mice to elucidate any metabolic pathways that are regulated by Nudt8 mediated CoA degradation. 


\section{References}

1. Leonardi, R., Zhang, Y. M., Rock, C. O., and Jackowski, S. (2005) Coenzyme A: back in action. Prog Lipid Res 44, 125-153

2. Guynn, R. W. R. R. W. (1973) Equilibrium constants of the malate dehydrogenase, citrate synthase, citrate lyase, and acetyl coenzyme A hydrolysis reactions under physiological conditions. Journal of Biological Chemistry 248, 6957-6965

3. Walsh, C. T., Tu, B. P., and Tang, Y. (2018) Eight Kinetically Stable but Thermodynamically Activated Molecules that Power Cell Metabolism. Chemical Reviews 118, 1460-1494

4. Choudhary, C., Weinert, B. T., Nishida, Y., Verdin, E., and Mann, M. (2014) The growing landscape of lysine acetylation links metabolism and cell signalling. Nature Reviews Molecular Cell Biology 15, 536-550

5. Zhang, Y., Bharathi, S. S., Rardin, M. J., Lu, J., Maringer, K. V., Sims-Lucas, S., Prochownik, E. V., Gibson, B. W., and Goetzman, E. S. (2017) Lysine desuccinylase SIRT5 binds to cardiolipin and regulates the electron transport chain. J Biol Chem 292, 10239-10249

6. Gout, I. (2018) Coenzyme A, protein CoAlation and redox regulation in mammalian cells. Biochem Soc Trans 46, 721-728

7. Cai, L., and Tu, B. P. (2011) On Acetyl-CoA as a Gauge of Cellular Metabolic State. Cold Spring Harbor Symposia on Quantitative Biology 76, 195-202

8. Pietrocola, F., Galluzzi, L., Bravo-San Pedro, J. M., Madeo, F., and Kroemer, G. (2015) Acetyl coenzyme A: a central metabolite and second messenger. Cell Metab 21, 805-821

9. Leonardi, R., Rehg, J. E., Rock, C. O., and Jackowski, S. (2010) Pantothenate kinase 1 is required to support the metabolic transition from the fed to the fasted state. PLoS One 5, e11107

10. Naquet, P., Kerr, E. W., Vickers, S. D., and Leonardi, R. (2020) Regulation of coenzyme A levels by degradation: the 'Ins and Outs'. Prog Lipid Res 78, 101028

11. Leonardi, R., Rock, C. O., and Jackowski, S. (2014) Pank1 deletion in leptin-deficient mice reduces hyperglycaemia and hyperinsulinaemia and modifies global metabolism without affecting insulin resistance. Diabetologia 57, 1466-1475

12. Zhang, Y. M., Chohnan, S., Virga, K. G., Stevens, R. D., Ilkayeva, O. R., Wenner, B. R., Bain, J. R., Newgard, C. B., Lee, R. E., Rock, C. O., and Jackowski, S. (2007) Chemical knockout of pantothenate kinase reveals the metabolic and genetic program responsible for hepatic coenzyme A homeostasis. Chem Biol 14, 291-302

13. Tokutake, Y., Onizawa, N., Katoh, H., Toyoda, A., and Chohnan, S. (2010) Coenzyme A and its thioester pools in fasted and fed rat tissues. Biochemical and Biophysical Research Communications 402, 158-162

14. Tokutake, Y., lio, W., Onizawa, N., Ogata, Y., Kohari, D., Toyoda, A., and Chohnan, S. (2012) Effect of diet composition on coenzyme $A$ and its thioester pools in various rat tissues. Biochemical and Biophysical Research Communications 423, 781-784

15. Garland, P., Shepherd, D., and Yates, D. (1965) Steady-state concentrations of coenzyme $A$, acetyl-coenzyme $A$ and long-chain fatty acyl-coenzyme $A$ in rat-liver mitochondria oxidizing palmitate. Biochemical Journal 97, 587-594

16. Smith, C. M., Cano, M. L., and Potyraj, J. (1978) The relationship between metabolic state and total CoA content of rat liver and heart. J Nutr 108, 854-862 
17. Van Broekhoven, A., Peeters, M.-C., Debeer, L. J., and Mannaerts, G. P. (1981) Subcellular distribution of coenzyme A: Evidence for a separate coenzyme a pool in peroxisomes. Biochemical and Biophysical Research Communications 100, 305-312

18. Siess, E. A., Brocks, D. G., and Wieland, O. H. (1978) Distribution of metabolites between the cytosolic and mitochondrial compartments of hepatocytes isolated from fed rats. Hoppe-Seyler's Zeitschrift fur physiologische Chemie 359, 785-798

19. Trefely, S., Lovell, C. D., Snyder, N. W., and Wellen, K. E. (2020) Compartmentalised acyl-CoA metabolism and roles in chromatin regulation. Mol Metab 38, 100941

20. Shumar, S. A., Kerr, E. W., Fagone, P., Infante, A. M., and Leonardi, R. (2019) Overexpression of Nudt7 decreases bile acid levels and peroxisomal fatty acid oxidation in the liver. J Lipid Res 60, 1005-1019

21. Peng, Y., and Puglielli, L. (2016) Ne-lysine acetylation in the lumen of the endoplasmic reticulum: A way to regulate autophagy and maintain protein homeostasis in the secretory pathway. Autophagy 12, 1051-1052

22. Longo, N., Frigeni, M., and Pasquali, M. (2016) Carnitine transport and fatty acid oxidation. Biochimica et biophysica acta 1863, 2422-2435

23. Palmieri, F. (2013) The mitochondrial transporter family SLC25: identification, properties and physiopathology. Mol Aspects Med 34, 465-484

24. Fiermonte, G., Paradies, E., Todisco, S., Marobbio, C. M., and Palmieri, F. (2009) A novel member of solute carrier family 25 (SLC25A42) is a transporter of coenzyme A and adenosine 3',5'-diphosphate in human mitochondria. J Biol Chem 284, 18152-18159

25. Prohl, C., Pelzer, W., Diekert, K., Kmita, H., Bedekovics, T., Kispal, G., and Lill, R. (2001) The yeast mitochondrial carrier Leu5p and its human homologue Graves' disease protein are required for accumulation of coenzyme A in the matrix. Mol Cell Biol 21, 1089-1097

26. Iuso, A., Alhaddad, B., Weigel, C., Kotzaeridou, U., Mastantuono, E., Schwarzmayr, T., Graf, E., Terrile, C., Prokisch, H., Strom, T. M., Hoffmann, G. F., Meitinger, T., and Haack, T. B. (2019) A Homozygous Splice Site Mutation in SLC25A42, Encoding the Mitochondrial Transporter of Coenzyme A, Causes Metabolic Crises and Epileptic Encephalopathy. JIMD Rep 44, 1-7

27. Shamseldin, H. E., Smith, L. L., Kentab, A., Alkhalidi, H., Summers, B., Alsedairy, H., Xiong, Y., Gupta, V. A., and Alkuraya, F. S. (2016) Mutation of the mitochondrial carrier SLC25A42 causes a novel form of mitochondrial myopathy in humans. Hum Genet 135, 21-30

28. Almannai, M., Alasmari, A., Alqasmi, A., Faqeih, E., Al Mutairi, F., Alotaibi, M., Samman, M. M., Eyaid, W., Aljadhai, Y. I., Shamseldin, H. E., Craigen, W., and Alkuraya, F. S. (2018) Expanding the phenotype of SLC25A42-associated mitochondrial encephalomyopathy. Clin Genet 93, 1097-1102

29. Zhyvoloup, A., Nemazanyy, I., Panasyuk, G., Valovka, T., Fenton, T., Rebholz, H., Wang, M. L., Foxon, R., Lyzogubov, V., Usenko, V., Kyyamova, R., Gorbenko, O., Matsuka, G., Filonenko, V., and Gout, I. T. (2003) Subcellular localization and regulation of coenzyme A synthase. J Biol Chem 278, 50316-50321

30. Rhee, H. W., Zou, P., Udeshi, N. D., Martell, J. D., Mootha, V. K., Carr, S. A., and Ting, A. Y. (2013) Proteomic mapping of mitochondria in living cells via spatially restricted enzymatic tagging. Science (New York, N.Y.) 339, 1328-1331

31. Dusi, S., Valletta, L., Haack, T. B., Tsuchiya, Y., Venco, P., Pasqualato, S., Goffrini, P., Tigano, M., Demchenko, N., Wieland, T., Schwarzmayr, T., Strom, T. M., Invernizzi, F., Garavaglia, B., Gregory, A., Sanford, L., Hamada, J., Bettencourt, C., Houlden, H., Chiapparini, L., Zorzi, G., Kurian, M. A., Nardocci, N., Prokisch, H., Hayflick, S., Gout, I., and Tiranti, V. (2014) Exome sequence reveals mutations in CoA synthase as a cause of neurodegeneration with brain iron accumulation. American journal of human genetics 94 , $11-22$ 
32. Baker, A., Carrier, D. J., Schaedler, T., Waterham, H. R., van Roermund, C. W., and Theodoulou, F. L. (2015) Peroxisomal ABC transporters: functions and mechanism. Biochem Soc Trans 43, 959-965

33. Agrimi, G., Russo, A., Scarcia, P., and Palmieri, F. (2012) The human gene SLC25A17 encodes a peroxisomal transporter of coenzyme A, FAD and NAD+. Biochem J 443, 241247

34. Kim, Y. I., Nam, I. K., Lee, D. K., Bhandari, S., Charton, L., Kwak, S., Lim, J. Y., Hong, K., Kim, S. J., Lee, J. N., Kwon, S. W., So, H. S., Linka, N., Park, R., and Choe, S. K. (2020) Slc25a17 acts as a peroxisomal coenzyme A transporter and regulates multiorgan development in zebrafish. J Cell Physiol 235, 151-165

35. Westin, M. A. K., Hunt, M. C., and Alexson, S. E. H. (2008) Short- and medium-chain carnitine acyltransferases and acyl-CoA thioesterases in mouse provide complementary systems for transport of $\beta$-oxidation products out of peroxisomes. Cellular and Molecular Life Sciences 65, 982-990

36. Le Borgne, F., Ben Mohamed, A., Logerot, M., Garnier, E., and Demarquoy, J. (2011) Changes in carnitine octanoyltransferase activity induce alteration in fatty acid metabolism. Biochem Biophys Res Commun 409, 699-704

37. Pehar, M., Lehnus, M., Karst, A., and Puglielli, L. (2012) Proteomic assessment shows that many endoplasmic reticulum (ER)-resident proteins are targeted by $\mathrm{N}$ (epsilon)-lysine acetylation in the lumen of the organelle and predicts broad biological impact. The Journal of biological chemistry 287, 22436-22440

38. Jonas, M. C., Pehar, M., and Puglielli, L. (2010) AT-1 is the ER membrane acetyl-CoA transporter and is essential for cell viability. J Cell Sci 123, 3378-3388

39. Peng, Y., Li, M., Clarkson, B. D., Pehar, M., Lao, P. J., Hillmer, A. T., Barnhart, T. E., Christian, B. T., Mitchell, H. A., Bendlin, B. B., Sandor, M., and Puglielli, L. (2014) Deficient import of acetyl-CoA into the ER lumen causes neurodegeneration and propensity to infections, inflammation, and cancer. J Neurosci 34, 6772-6789

40. Peng, Y., Shapiro, S. L., Banduseela, V. C., Dieterich, I. A., Hewitt, K. J., Bresnick, E. H., Kong, G., Zhang, J., Schueler, K. L., Keller, M. P., Attie, A. D., Hacker, T. A., Sullivan, R., Kielar-Grevstad, E., Arriola Apelo, S. I., Lamming, D. W., Anderson, R. M., and Puglielli, L. (2018) Increased transport of acetyl-CoA into the endoplasmic reticulum causes a progeria-like phenotype. Aging cell 17, e12820

41. Hullinger, R., Li, M., Wang, J., Peng, Y., Dowell, J. A., Bomba-Warczak, E., Mitchell, H. A., Burger, C., Chapman, E. R., Denu, J. M., Li, L., and Puglielli, L. (2016) Increased expression of AT-1/SLC33A1 causes an autistic-like phenotype in mice by affecting dendritic branching and spine formation. The Journal of experimental medicine 213, 12671284

42. Rock, C. O., Calder, R. B., Karim, M. A., and Jackowski, S. (2000) Pantothenate kinase regulation of the intracellular concentration of coenzyme A. J Biol Chem 275, 1377-1383

43. Rock, C. O., Karim, M. A., Zhang, Y. M., and Jackowski, S. (2002) The murine pantothenate kinase (Pank1) gene encodes two differentially regulated pantothenate kinase isozymes. Gene 291, 35-43

44. Leonardi, R., Zhang, Y. M., Lykidis, A., Rock, C. O., and Jackowski, S. (2007) Localization and regulation of mouse pantothenate kinase 2. FEBS Lett 581, 4639-4644

45. Alfonso-Pecchio, A., Garcia, M., Leonardi, R., and Jackowski, S. (2012) Compartmentalization of mammalian pantothenate kinases. PloS one 7, e49509-e49509

46. Kuo, Y. M., Duncan, J. L., Westaway, S. K., Yang, H., Nune, G., Xu, E. Y., Hayflick, S. J., and Gitschier, J. (2005) Deficiency of pantothenate kinase 2 (Pank2) in mice leads to retinal degeneration and azoospermia. Hum Mol Genet 14, 49-57

47. Kurian, M. A., and Hayflick, S. J. (2013) Chapter Two - Pantothenate Kinase-Associated Neurodegeneration (PKAN) and PLA2G6-Associated Neurodegeneration (PLAN): Review 
of Two Major Neurodegeneration with Brain Iron Accumulation (NBIA) Phenotypes. in International Review of Neurobiology (Bhatia, K. P., and Schneider, S. A. eds.), Academic Press. pp 49-71

48. Zhang, Y. M., Rock, C. O., and Jackowski, S. (2005) Feedback regulation of murine pantothenate kinase 3 by coenzyme A and coenzyme A thioesters. J Biol Chem 280, 32594-32601

49. Sharma, L. K., Subramanian, C., Yun, M.-K., Frank, M. W., White, S. W., Rock, C. O., Lee, R. E., and Jackowski, S. (2018) A therapeutic approach to pantothenate kinase associated neurodegeneration. Nature Communications 9, 4399

50. Daugherty, M., Polanuyer, B., Farrell, M., Scholle, M., Lykidis, A., de Crécy-Lagard, V., and Osterman, A. (2002) Complete Reconstitution of the Human Coenzyme A Biosynthetic Pathway via Comparative Genomics. Journal of Biological Chemistry 277, 21431-21439

51. Zhyvoloup, A., Nemazanyy, I., Babich, A., Panasyuk, G., Pobigailo, N., Vudmaska, M., Naidenov, V., Kukharenko, O., Palchevskii, S., Savinska, L., Ovcharenko, G., Verdier, F., Valovka, T., Fenton, T., Rebholz, H., Wang, M. L., Shepherd, P., Matsuka, G., Filonenko, V., and Gout, I. T. (2002) Molecular cloning of CoA Synthase. The missing link in CoA biosynthesis. J Biol Chem 277, 22107-22110

52. Nemazanyy, I., Panasyuk, G., Breus, O., Zhyvoloup, A., Filonenko, V., and Gout, I. T. (2006) Identification of a novel CoA synthase isoform, which is primarily expressed in the brain. Biochem Biophys Res Commun 341, 995-1000

53. Gudkova, D., Panasyuk, G., Nemazanyy, I., Zhyvoloup, A., Monteil, P., Filonenko, V., and Gout, I. (2012) EDC4 interacts with and regulates the dephospho-CoA kinase activity of CoA synthase. FEBS Lett 586, 3590-3595

54. Arif, A., Jia, J., Willard, B., Li, X., and Fox, P. L. (2019) Multisite Phosphorylation of S6K1 Directs a Kinase Phospho-code that Determines Substrate Selection. Mol Cell 73, 446457.e446

55. Nemazanyy, I., Panasyuk, G., Zhyvoloup, A., Panayotou, G., Gout, I. T., and Filonenko, V. (2004) Specific interaction between S6K1 and CoA synthase: a potential link between the mTOR/S6K pathway, CoA biosynthesis and energy metabolism. FEBS Letters 578, 357-362

56. Breus, O., Panasyuk, G., Gout, I. T., Filonenko, V., and Nemazanyy, I. (2010) CoA Synthase is phosphorylated on tyrosines in mammalian cells, interacts with and is dephosphorylated by Shp2PTP. Molecular and Cellular Biochemistry 335, 195-202

57. Ferrandon, S., DeVecchio, J., Duraes, L., Chouhan, H., Karagkounis, G., Davenport, J., Orloff, M., Liska, D., and Kalady, M. F. (2020) CoA Synthase (COASY) Mediates Radiation Resistance via PI3K Signaling in Rectal Cancer. Cancer Res 80, 334-346

58. Breus, O., Panasyuk, G., Gout, I. T., Filonenko, V., and Nemazanyy, I. (2009) CoA Synthase is in complex with p85aPI3K and affects PI3K signaling pathway. Biochemical and Biophysical Research Communications 385, 581-585

59. Lin, C. C., Kitagawa, M., Tang, X., Hou, M. H., Wu, J., Qu, D. C., Srinivas, V., Liu, X., Thompson, J. W., Mathey-Prevot, B., Yao, T. P., Lee, S. H., and Chi, J. T. (2018) CoA synthase regulates mitotic fidelity via CBP-mediated acetylation. Nat Commun 9, 1039

60. Corbin, D. R., Rehg, J. E., Shepherd, D. L., Stoilov, P., Percifield, R. J., Horner, L., Frase, S., Zhang, Y. M., Rock, C. O., Hollander, J. M., Jackowski, S., and Leonardi, R. (2017) Excess coenzyme A reduces skeletal muscle performance and strength in mice overexpressing human PANK2. Mol Genet Metab 120, 350-362

61. Leoni, V., Strittmatter, L., Zorzi, G., Zibordi, F., Dusi, S., Garavaglia, B., Venco, P., Caccia, C., Souza, A. L., Deik, A., Clish, C. B., Rimoldi, M., Ciusani, E., Bertini, E., Nardocci, N., Mootha, V. K., and Tiranti, V. (2012) Metabolic consequences of mitochondrial coenzyme A deficiency in patients with PANK2 mutations. Mol Genet Metab 105, 463-471 
62. Stipanuk, M. H., Dominy, J. E., Jr., Lee, J.-I., and Coloso, R. M. (2006) Mammalian Cysteine Metabolism: New Insights into Regulation of Cysteine Metabolism. The Journal of Nutrition 136, 1652S-1659S

63. McLennan, A. G. (2006) The Nudix hydrolase superfamily. Cellular and molecular life sciences : CMLS 63, 123-143

64. Mildvan, A. S., Xia, Z., Azurmendi, H. F., Saraswat, V., Legler, P. M., Massiah, M. A., Gabelli, S. B., Bianchet, M. A., Kang, L. W., and Amzel, L. M. (2005) Structures and mechanisms of Nudix hydrolases. Archives of Biochemistry and Biophysics 433, 129-143

65. AbdelRaheim, S. R., and McLennan, A. G. (2002) The Caenorhabditis elegans Y87G2A.14 Nudix hydrolase is a peroxisomal coenzyme A diphosphatase. BMC Biochem $3,5-5$

66. Cartwright, J. L., Gasmi, L., Spiller, D. G., and McLennan, A. G. (2000) The Saccharomyces cerevisiae PCD1 Gene Encodes a Peroxisomal Nudix Hydrolase Active toward Coenzyme A and Its Derivatives. Journal of Biological Chemistry 275, 3292532930

67. Gasmi, L., and McLennan, A. G. (2001) The mouse Nudt7 gene encodes a peroxisomal nudix hydrolase specific for coenzyme A and its derivatives. The Biochemical journal 357, 33-38

68. Ito, D., Yoshimura, K., Ishikawa, K., Ogawa, T., Maruta, T., and Shigeoka, S. (2012) A Comparative Analysis of the Molecular Characteristics of the Arabidopsis CoA Pyrophosphohydrolases AtNUDX11, 15, and 15a. Bioscience, Biotechnology, and Biochemistry 76, 139-147

69. Kang, L.-W., Gabelli, S. B., Bianchet, M. A., Xu, W. L., Bessman, M. J., and Amzel, L. M. (2003) Structure of a coenzyme A pyrophosphatase from Deinococcus radiodurans: a member of the Nudix family. J Bacteriol 185, 4110-4118

70. Ofman, R., Speijer, D., Leen, R., and Wanders, R. J. A. (2006) Proteomic analysis of mouse kidney peroxisomes: identification of RP2 $p$ as a peroxisomal nudix hydrolase with acyl-CoA diphosphatase activity. The Biochemical journal 393, 537-543

71. Spangler, J. R., and Huang, F. (2020) The <em>E. coli</em> NudL enzyme is a Nudix hydrolase that cleaves CoA and its derivatives. bioRxiv, 2020.2001.2031.929182

72. Shumar, S. A., Kerr, E. W., Geldenhuys, W. J., Montgomery, G. E., Fagone, P., Thirawatananond, P., Saavedra, H., Gabelli, S. B., and Leonardi, R. (2018) Nudt19 is a renal CoA diphosphohydrolase with biochemical and regulatory properties that are distinct from the hepatic Nudt7 isoform. J Biol Chem 293, 4134-4148

73. Reilly, S.-J., Tillander, V., Ofman, R., Alexson, S. E. H., and Hunt, M. C. (2008) The Nudix Hydrolase 7 is an Acyl-CoA Diphosphatase Involved in Regulating Peroxisomal Coenzyme A Homeostasis. The Journal of Biochemistry 144, 655-663

74. Kirkby, B., Roman, N., Kobe, B., Kellie, S., and Forwood, J. K. (2010) Functional and structural properties of mammalian acyl-coenzyme A thioesterases. Progress in Lipid Research 49, 366-377

75. Tillander, V., Alexson, S. E. H., and Cohen, D. E. (2017) Deactivating Fatty Acids: AcylCoA Thioesterase-Mediated Control of Lipid Metabolism. Trends in endocrinology and metabolism: TEM 28, 473-484

76. Ellis, J. M., Bowman, C. E., and Wolfgang, M. J. (2015) Metabolic and tissue-specific regulation of acyl-CoA metabolism. PloS one 10, e0116587-e0116587

77. Bekeova, C., Anderson-Pullinger, L., Boye, K., Boos, F., Sharpadskaya, Y., Herrmann, J. M., and Seifert, E. L. (2019) Multiple mitochondrial thioesterases have distinct tissue and substrate specificity and CoA regulation, suggesting unique functional roles. Journal of Biological Chemistry 294, 19034-19047 


\section{Chapter II: Biochemical characterization of Nudt8}

Kerr, E.W., S.A. Shumar, and R. Leonardi, Nudt8 is a novel CoA diphosphohydrolase that resides in the mitochondria. FEBS Lett, 2019. 593(11): p. 1133-1143.

Author contributions: Evan W. Kerr and Roberta Leonardi designed the experiments. Evan W. Kerr and Stephanie A. Shumar performed the experiments. Evan W. Kerr, Stephanie A. Shumar and Roberta Leonardi wrote the manuscript.

This research was origionally published in FEBS Letters. Kerr, E.W., S.A. Shumar, and R. Leonardi, Nudt8 is a novel CoA diphosphohydrolase that resides in the mitochondria. FEBS Lett, 2019. 593(11): p. 1133-1143.

(C) 2019 Federation of European Biochemical Societies 


\section{Abstract}

Coenzyme $A(\mathrm{Co} A)$ regulates energy metabolism and exists in separate pools in the cytosol, peroxisomes, and mitochondria. At the whole tissue level, the concentration of CoA changes with the nutritional state by balancing synthesis and degradation; however, it is currently unclear how individual subcellular CoA pools are regulated. Liver and kidney peroxisomes contain Nudt7 and Nudt19, respectively, enzymes that catalyze CoA degradation. We report that Nudt8 is a novel CoA-degrading enzyme that resides in the mitochondria. Nudt8 has a distinctive preference for manganese ions and exhibits a broader tissue distribution than Nudt7 and Nudt19. The existence of CoA-degrading enzymes in both peroxisomes and mitochondria suggests that degradation may be a key regulatory mechanism for modulating the intracellular CoA pools.

\section{Introduction}

CoA is an indispensable cofactor that activates acyl groups for a multitude of metabolic reactions and the widespread posttranslational acylation of histones and other proteins (1-5). Unacylated CoA (free CoA) has also been found to directly react with cysteine residues of target proteins under conditions of metabolic stress, resulting in protein ‘CoAlation' (6). CoA-dependent metabolism and post-translational modifications occur in multiple subcellular compartments, including the nucleus and the endoplasmic reticulum, but the cytosol, peroxisomes, and mitochondria contain the major pools of this cofactor $(5,7-9)$. The mitochondria are estimated to contain the highest concentration of $\operatorname{CoA}(7,8,10)$, which is required to activate gluconeogenesis and for pathways such as ketogenesis, fatty acid oxidation, and the TCA cycle.

At the whole tissue level, the concentration of CoA changes with the metabolic state by coordinating synthesis and degradation. In the liver, activation of the biosynthetic pathway drives the increase in CoA that occurs in the transition between the fed and fasted state $(11,12)$. Conversely, the net decrease in the concentration of hepatic CoA observed upon refeeding after a fast requires both inhibition of de novo CoA synthesis and degradation of the cofactor 
accumulated in the fasted state. At the subcellular level, such metabolic adaptations require modulation of the size and composition of the individual CoA pools $(7,8)$. To date, however, our knowledge of the mechanisms regulating these subcellular CoA pools is limited.

In the peroxisomes of liver and kidneys, CoA is hydrolyzed to 3',5'-ADP and (acyl)phosphopantetheine by two Nudix hydrolases, Nudt7 and Nudt19, respectively (13-16). Unlike intact CoA, these smaller degradation products can exit the peroxisomes (17); thus, the activity of Nudt7 and Nudt19 likely plays an important role in the regulation of the peroxisomal CoA pool and, in turn, CoA-dependent peroxisomal metabolism $(12,17,18)$. Consistent with such a role, the expression of Nudt7 and the activity of Nudt19 are higher in the fed state compared to the fasted state, and inversely correlate with tissue CoA levels in the liver and kidneys, respectively $(13,16,19)$.

Nudix hydrolases hydrolyze phosphodiester bonds in a variety of substrates including nucleoside di- and triphosphates, dinucleoside polyphosphates, nucleotide sugars and cofactors like CoA and $\operatorname{NAD}(P) H(20,21)$. All Nudix hydrolases are characterized by the presence of the Nudix box motif, G[5X]E[7X]REXXEEXGU (where $U$ is a hydrophobic residue and $X$ is any residue), which coordinates a catalytically essential divalent cation $(22,23)$. Nudix hydrolases that specifically hydrolyze CoA contain another conserved motif, $(\mathrm{L} / \mathrm{M})(\mathrm{L} / \mathrm{F}) \mathrm{TXR}(\mathrm{S} / \mathrm{A})[3 \mathrm{X}](\mathrm{R} / \mathrm{K})[3 \mathrm{X}] \mathrm{G}[3 \mathrm{X}] \mathrm{FPGG}$ (PROSITE accession number PS01293), called the CoA box, which contains residues important for CoA binding and catalysis $(13,24)$. In addition to mammals, peroxisomal Nudix hydrolases specific for CoA are present in yeast and nematodes $(24,25)$. Plants contain cytosolic and mitochondrial isoforms (26); however, no mammalian counterparts have been identified so far.

Nudt8 is an uncharacterized Nudix hydrolase that has been annotated as a mitochondrial enzyme and is closely related to Nudt7 $(21,27)$. In this study, we show that Nudt8 specifically hydrolyzes CoA, is indeed a mitochondrial enzyme, and is broadly expressed in mitochondria-rich organs such as the heart, kidneys, liver, and brown adipose tissue. The characterization of the 
first mitochondrial CoA-degrading enzyme in mammals provides a potential mechanism for the dynamic regulation of the mitochondrial CoA pool.

\section{Materials and Methods}

\section{Materials}

Reagents were purchased from the following suppliers: oligonucleotides, Lipofectamine 2000, cell culture reagents, Alexa Fluor 647- and HRP-conjugated goat anti-rabbit IgG, and MitoTracker Orange CMTMRos from Thermo Fisher Scientific; restriction enzymes from New England Biolabs; HEK293 and HEK293T cells from the American Type Culture Collection; Swift membrane stain from G-biosciences. Antibodies against glyceraldehyde-3-phosphate dehydrogenase (GAPDH), citrate synthase, and catalase were purchased from Cell Signaling. The Nudt7 antibody was generated as previously described (19). 3',5'-ADP and antibodies against ATP-binding cassette, sub-family D, member 3 (PMP70) and the FLAG epitope were obtained from Sigma-Aldrich; the Dylight 488 mouse lgG was purchased from Rockland Immunochemicals. The antibody against the full-length mouse Nudt8 was raised in rabbit and purified by antigen affinity chromatography. The fluorescent CoA derivative of monobromobimane (mBB), mBB-CoA, was synthesized as previously described (13). All other reagents were of analytical grade or better and were purchased from Sigma-Aldrich or Fisher Scientific, unless otherwise stated.

\section{Nudt8 purification and enzymatic assays}

Mouse Nudt8 (accession NP_079805.1) was expressed as a C-terminal hexahistinetagged protein from the pET21-derived plasmid pKM202, a kind gift of Suzanne Jackowski at St. Jude Children's Research Hospital. Nudt8 was expressed in E.coli BL21 Codon Plus (DE3)-RIPL cultured in auto-inducing medium for $24 \mathrm{~h}$ at room temperature (28). The enzyme was purified by Ni-NTA chromatography, followed by dialysis in $20 \mathrm{mM}$ Tris-HCl, pH 8.5, 1 mM EDTA, 1 mM DTT 
at $4{ }^{\circ} \mathrm{C}$ overnight. The combined dialyzed fractions were applied onto a POROS $50 \mathrm{HQ}$ column (Thermo Fisher Scientific). Nudt8 was eluted in two peaks at 160 and $305 \mathrm{mM} \mathrm{NaCl}$ in $40 \mathrm{mM}$ diethanolamine hydrochloride, $\mathrm{pH} 9.0$. Only the peak eluted at $160 \mathrm{mM} \mathrm{NaCl}$ contained enzymatic activity. Active fractions containing $>80 \%$ pure Nudt8 were combined and concentrated to $>1$ $\mathrm{mg} / \mathrm{ml}$. Glycerol was added to a final concentration of $50 \%$ before storing the protein at $-20{ }^{\circ} \mathrm{C}$. For the analysis of Nudt8 by analytical gel filtration, purified recombinant Nudt8 $(50 \mu l)$ was loaded onto a Superdex 75 10/300 GL column and eluted at $0.3 \mathrm{ml} / \mathrm{min}$ with $50 \mathrm{mM}$ Tris- $\mathrm{HCl}, 150 \mathrm{~mm}$ $\mathrm{NaCl}, 1 \mathrm{mM}$ DTT, $1 \mathrm{mM}$ EDTA, $\mathrm{pH}$ 8.5. Protein elution was monitored by measuring the absorbance at $280 \mathrm{~nm}$. The activity of Nudt8 was assayed as previously described (13). The optimized standard assay mixture contained $50 \mathrm{ng}$ of enzyme, $100 \mathrm{mM}$ Tris-HCl pH 8.5, $0.1 \mathrm{mg} / \mathrm{ml}$ v-globulin, $250 \mu \mathrm{M}$ of free CoA or acyl-CoA substrates and $2 \mathrm{mM} \mathrm{MnCl}$, in a total volume of 40 $\mu \mathrm{l}$. Kinetic parameters were determined by varying the substrate concentration from 0.125 to 4 $\mathrm{mM}$. Data points, individually corrected for the background in reaction mixtures lacking the enzyme, were fitted to a 'Specific Binding with Hill slope' and 'kcat' models using Graphpad Prism 6 (GraphPad Software) to determine the kinetic parameters.

\section{Animal studies}

C57BL/6J male mice were purchased from the Jackson Laboratory and allowed to acclimate for at least 7 days. The mice were fed a standard chow diet (Tekland 2018S) and maintained at a room temperature of $22.2 \pm 0.2{ }^{\circ} \mathrm{C}$, room humidity of $40 \% \pm 2 \%$, and a 12-h light, 12-h dark cycle, with the dark cycle starting at 6:00 pm. For fasting experiments, the mice were placed on cages with grids for $24 \mathrm{~h}$ without food. All studies were approved by the Institutional Animal Care and Use Committees of West Virginia University.

\section{Subcellular fractionation}


All centrifugation steps were conducted at $4{ }^{\circ} \mathrm{C}$. To obtain mitochondria-free peroxisomal fractions, we pooled the livers from two mice and adapted the procedure described by Antonenkov, V. D. et. al. (29). Briefly, the combined livers were weighed, minced, and homogenized in 4 volumes of peroxisomal homogenization medium ( $0.16 \mathrm{M}$ sucrose, $12 \%$ PEG1500, 10 mM MOPS pH 7.4, 1 mM EDTA, 1 mM EGTA, 0.1\% ethanol, 0.1 mM PMSF, 2 mM DTT) using 5-6 strokes of a motor-driven Teflon pestle at a speed of $550 \mathrm{rpm}$. The homogenate was centrifuged at $1,500 \times \mathrm{g}$ for $12 \mathrm{~min}$, and the supernatant was applied onto a $50 \%$ Percoll cushion. After centrifuging at $23,500 \times g$ for $1 \mathrm{~h}$, the peroxisomal fraction was removed from the bottom of the cushion, diluted with $1 \mathrm{ml}$ of homogenization medium, and applied onto an Optiprep density gradient. The gradient was centrifuged at $68,000 \times g$ in a $70.1 \mathrm{Ti}$ fixed angle rotor for $1 \mathrm{~h}$. Fractions $(1 \mathrm{ml})$ were removed starting from the bottom of the Optiprep gradient and stored at $80^{\circ} \mathrm{C}$ until analyzed.

\section{Immunoblotting, RT-PCR and immunofluorescence}

For western blot analysis, subcellular fractions and tissue homogenates were fractionated by SDS-PAGE on $4-12 \%$ Bis-Tris gels and transferred onto PVDF membranes. Flash frozen tissues were homogenized in ice-cold radioimmunoprecipitation assay buffer containing protease inhibitors and centrifuged at $10,000 \times \mathrm{g}$ for $10 \mathrm{~min}$ at $4^{\circ} \mathrm{C}$. To normalize antibody signals to total protein loaded, membranes were stained with Swift membrane stain as per manufacturer's instructions. Antibodies were used at the following dilutions: Nudt8, Nudt7 and GAPDH antibodies at 1:3,000; citrate synthase, catalase and FLAG antibodies at 1:1,000. Primary antibodies were detected by chemiluminescence with HRP-conjugated goat anti-rabbit or goat anti-mouse $\lg G$ at 1:45,000 dilution. Western blot bands were quantified using Genetools (Syngene) or ImageJ analysis software.

RNA was isolated from flash frozen tissue as previously described $(13,30)$. Nudt8 mRNA levels were quantified in triplicate by RT-PCR using the Quantitect SYBR Green RT-PCR kit 
(Qiagen) and the primers 5'-CAGTTTCCCAGGCGGTAAGT-3' and 5'CACGTTGGCAAGTACTGGGA-3' as the forward and reverse primers, respectively. Primers for Nudt7 and Nudt19 have been published (30). For each tissue, the relative abundance of Nudt7, Nudt8, and Nudt19 mRNA was calculated by the CT method using the averaged CT values for two calibrators, ribosomal protein L22 (Rpl22) and $\beta 2$-microglobulin (B2m). Primers 5'GTGCCTTTCTCCAAAAGGTATTT-3' and 5'-CTCTCTTTGCTGTTGGCGAC-3' were used as forward and reverse primers, respectively, for Rpl22. Primers 5'-ACTGACCGGCCTGTATGCTA3' and 5'-ATGTTCGGCTTCCCATTCTCC-3' were used as forward and reverse primers, respectively for $\mathrm{B} 2 \mathrm{~m}$.

To express Nudt8 in mammalian cells, Nudt8 was amplified from pKM202 using primers introducing an EcoRI restriction site and a Kozak sequence at the 5' end and a FLAG tag and an Xho/ restriction site at the $3^{\prime}$ end. The amplified product was subcloned into pcDNA3.1(+) (Thermo Fisher Scientific) between EcoRI and Xhol sites. HEK293 and HEK293T cells were cultured and transfected as previously described (13). The localization of mouse Nudt8 in HEK293 cells was determined by confocal microscopy, as previously reported (13). The FLAG antibody was used at a 1:1,000 dilution and incubated overnight. Dylight 488 goat anti-mouse IgG was used at a $1: 5,000$ dilution.

\section{Statistical Analysis}

Unless otherwise stated, all data are reported as the mean \pm standard error. Statistical significance was calculated by unpaired two-tailed Student's $t$ test using GraphPad Prism 6 (GraphPad Software). 
$\underline{\text { Results }}$

Nudt8 specifically hydrolyzes CoA species and has a distinctive preference for manganese ions

Mouse Nudt8 shares a 38\% sequence identity with the CoA-degrading enzyme Nudt7 (Fig

2.1A). Both proteins contain the CoA and Nudix boxes and the sequence identity in these regions

increases to $60 \%$. To test the

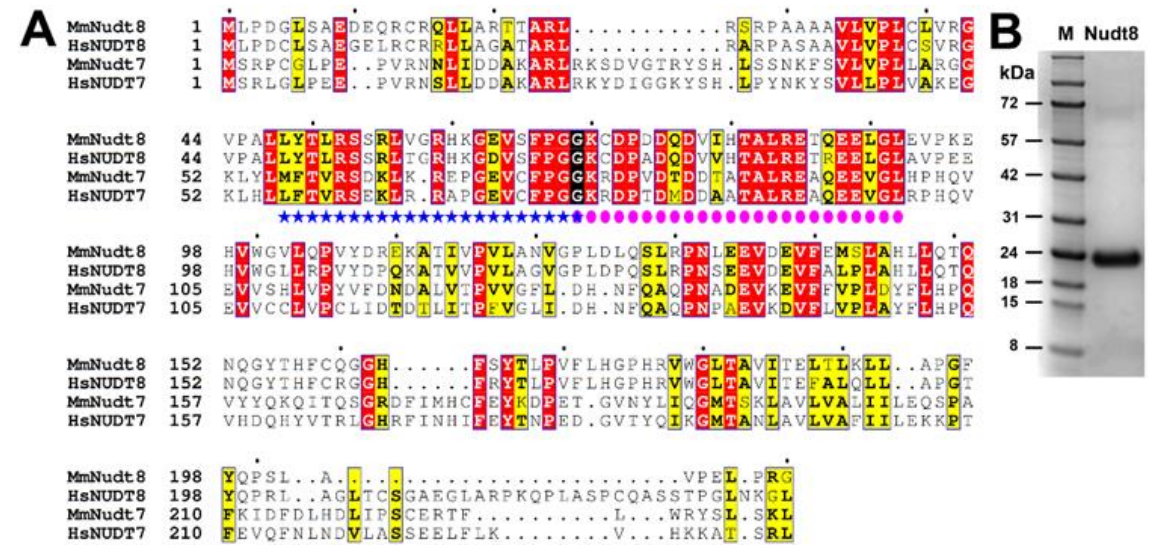

hexahistidine tag (Fig. 2.1B).

Compared to recombinant Nudt7,

Nudt8 was purified in significantly

lower amounts. Indeed, one liter of

auto-induced culture typically

yielded about $15 \mathrm{mg}$ of Nudt8

compared to the $70-100 \mathrm{mg}$ of
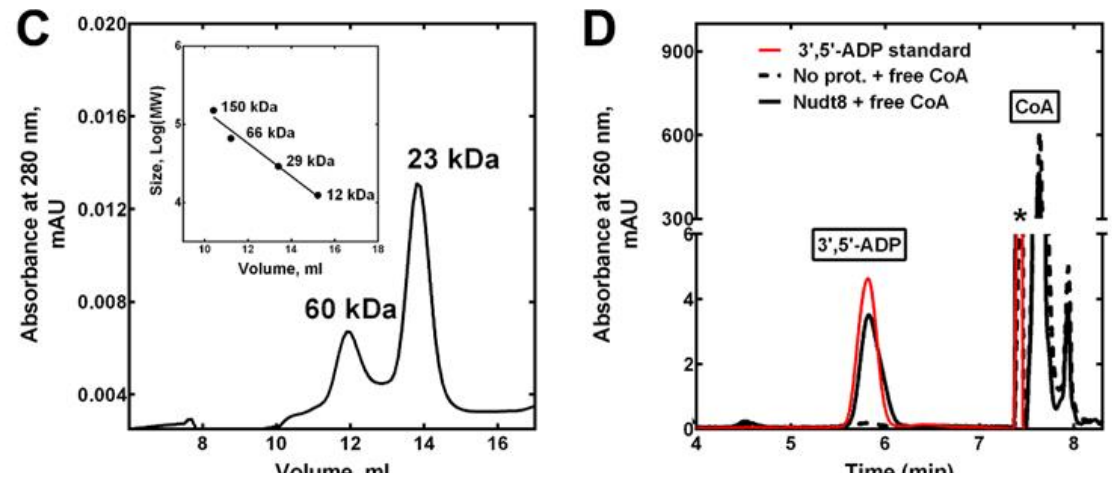

Figure 2.1 Expression and characterization of mouse Nudt8. (A)

Alignment of the protein sequences of mouse and human Nudt8 and Nudt7. Invariant residues are highlighted in red, conserved residues in

Nudt7 obtained per liter of yellow, and groups of conserved residues are boxed. The Nudix box (blue stars) and the CoA box (pink circles) overlap at a glycine highlighted in standard E. coli culture (13).

Interestingly, we also attempted

to express human NUDT8;

however, similar to a report by

Carreras-Puigvert et al., we could

not detect any expression (21).

Analytical gel filtration analysis of

black. Mm, Mus musculus; Hs, Homo sapiens; Genbank Gl numbers for the aligned proteins are: MmNudt8, 13384950; HsNUDT8, 344217763; MmNudt7, 165972342; HsNUDT7, 157785656. Sequence alignments were obtained using ESPript 3 (1). (B) Coomassie blue-stained protein gel showing purified Nudt8. M, molecular weight marker. (C) Elution profile of purified, His-tagged Nudt8 from a Superdex 75 10/300 GL column. The inset shows the calibration curve used to estimate the apparent molecular weight of Nudt8. The calculated molecular weight of the recombinant protein, based on the amino acid composition, is $24.3 \mathrm{kDa}$. (D) Highperformance liquid chromatography (HPLC) elution profile of the degradation product co-migrating with a 3',5'-ADP standard formed in reaction mixtures containing Nudt8 and free CoA. * marks a solvent impurity. 
recombinant mouse Nudt8 indicated that similar to Nudt7 (13), about $70 \%$ of the protein eluted as a monomer (Fig. 2.1C).

We next tested the hydrolytic activity of Nudt8 in the presence of $4 \mathrm{mM} \mathrm{MgCl}_{2}$ a nd a broad range of Nudix hydrolase substrates including ATP, ADP, NAD+, NADH, NADP+, NADPH, diadenosine triphosphate, ADP-ribose and free CoA. Product formation was detected only with free CoA as a substrate and co-elution of this product with a 3',5'-ADP standard confirmed that Nudt8 was a CoA diphosphohydrolase (Fig. 2.1D). Under the assay conditions used for Nudt7 (13), the specific activity of Nudt8 was about 100-fold lower (Fig. 2.2B), suggesting that one or more components of the reaction mixture needed to be optimized for Nudt8. Raising the buffer pH from 8.0 to 8.5 modestly increased Nudt8 activity; however, substituting $\mathrm{Mg}^{2+}$ with $\mathrm{Mn}^{2+}$ as the catalytically essential divalent cation in the reaction mixture, increased free CoA hydrolysis from about $2 \%$ to over $90 \%$ (Fig. $2.1 \mathrm{~A})$.
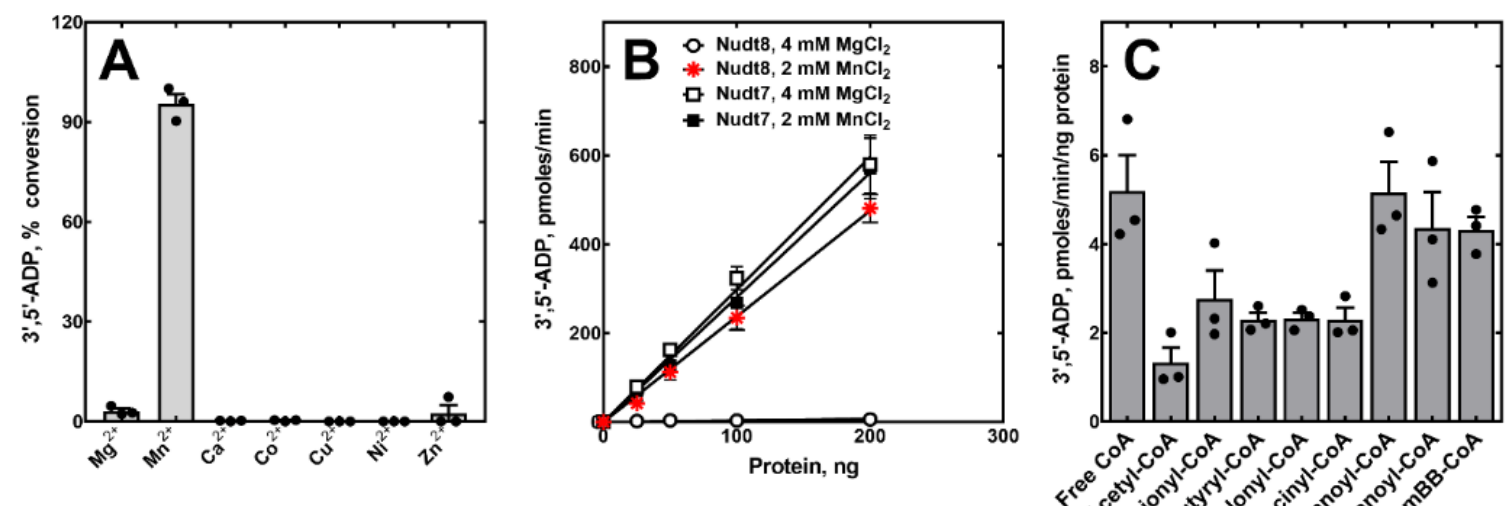

Figure 2.2. Divalent cation requirement and substrate specificity of recombinant Nudt8. (A) The ability of different divalent cations to support the hydrolytic activity of Nudt8 was tested in reaction mixtures containing $6 \mu \mathrm{g}$ of Nudt8, $250 \mu \mathrm{M}$ of free CoA and $0.5 \mathrm{mM}$ of each cation introduced as the chloride salt. (B) Nudt8 and Nudt7 protein curves in the presence of the indicated concentrations of $\mathrm{MgCl}_{2}$ and $\mathrm{MnCl}_{2}$. Linear regression of the curves, obtained using 3 independently purified Nudt8 batches in duplicate, was used to calculate the specific activity of the enzymes \pm standard error. In the presence of the optimal concentration of $\mathrm{MnCl}_{2}$, the specific activity of Nudt8 $(2.4 \pm 0.1 \mathrm{pmoles} / \mathrm{min} / \mathrm{ng}$ of protein) was comparable to the specific activity of Nudt7 with either $\mathrm{MgCl}_{2}\left(3.0 \pm 0.1 \mathrm{pmoles} / \mathrm{min} / \mathrm{ng}\right.$ of protein) or $\mathrm{MnCl}_{2}(2.8 \pm 0.1 \mathrm{pmles} / \mathrm{min} / \mathrm{ng}$ of protein). (C) CoA diphosphohydrolase activity of Nudt8 against multiple CoA substrates. Data in panels $A$ and $C$ are plotted as the mean (bars) of experiments conducted in duplicate using 3 independently purified Nudt8 batches (circles) \pm SEM. 
The optimal concentration of $\mathrm{MnCl}_{2}$ was found to be $2 \mathrm{mM}$ (data not shown). Under these optimized conditions, the specific activity of Nudt8 against free CoA was comparable to that of Nudt7 with either $\mathrm{MgCl}_{2}$ or $\mathrm{MnCl}_{2}$ (Fig. 2.2B). Importantly, we re-screened the diphosphatecontaining molecules above as potential Nudt8 substrates in the presence of $2 \mathrm{mM} \mathrm{MnCl}_{2}$ and confirmed that the activity of the enzyme remained specific for free CoA (data not shown).

Having established that Nudt8 was a CoA diphosphohydrolase, we measured its activity

\begin{tabular}{lcccc} 
against various short- and medium- & Substrate & $\mathbf{K}_{\mathbf{M}}, \boldsymbol{\mu M}$ & $\boldsymbol{k}_{\text {cat }}, \mathbf{m i n}^{-\mathbf{1}}$ & $\boldsymbol{k}_{\text {cat }} / \mathbf{K}_{\mathbf{M}}, \mathbf{m i n}^{-\mathbf{1 *}} \boldsymbol{\mu M}^{\mathbf{1}}$ \\
\cline { 2 - 5 } chain acyl-CoAs. We could not test & Free CoA & $150 \pm 13$ & $216 \pm 8$ & 1.44 \\
long-chain acyl-CoAs, such as & Acetyl-CoA & $707 \pm 252$ & $116 \pm 10$ & 0.16 \\
lauroyl- and stearoyl-CoA, as these & Propionyl-CoA & $318 \pm 87$ & $155 \pm 14$ & 0.49 \\
compounds precipitated even at the & Malonyl-CoA & $233 \pm 71$ & $184 \pm 19$ & 0.48 \\
lowest concentration of $\mathrm{MnCl}_{2}$ & Succinyl-CoA & $329 \pm 72$ & $187 \pm 14$ & 0.79 \\
required to detect Nudt8 activity. & Hexanoyl-CoA & $251 \pm 98$ & $149 \pm 11$ & 0.57 \\
\cline { 2 - 5 } & & &
\end{tabular}

Nudt8 hydrolyzed all CoA species

Table 2.1. Kinetic Parameters of Nudt8. $K_{M}, k_{c a t}$ and specificity constant $\left(k_{c a t} / K_{M}\right)$ for Nudt8 with different CoA substrates. The kinetic parameters $\mathrm{K}_{\mathrm{M}}$ and $k_{\text {cat }}$ were tested, exhibiting the highest activity toward free CoA, hexanoyl- and determined, \pm SEM, by non-linear regression analysis of data obtained from 3 independent experiments, each conducted in duplicate.

octanoyl-CoA and the lowest activity against acetyl-CoA (Fig. 2.2 C). Nudt8 also readily hydrolyzed the synthetic CoA derivative mBB-CoA. Analysis of Nudt8 kinetics toward representative acyl-CoAs revealed $\mathrm{K}_{\mathrm{MS}}$ ranging from $150 \mu \mathrm{M}$ for free CoA to $707 \mu \mathrm{M}$ for acetylCoA (Table 2.1). The values were in the same range of KMs reported for Nudt7 and Nudt19 (1416). Taking both the $k_{c a t}$ and the $K_{M}$ into consideration, the specificity constants calculated for the substrates confirmed that free CoA and acetyl-CoA were the best and worst substrates for Nudt8, respectively (Table 2.1).

Combined, these data indicated that Nudt8 is a novel CoA diphosphohydrolase with broad acyl-CoA substrate specificity and a distinctive preference for $\mathrm{Mn}^{2+}$, a feature not observed in the other known mammalian isoforms, Nudt7 and Nudt19 $(14,15)$. 

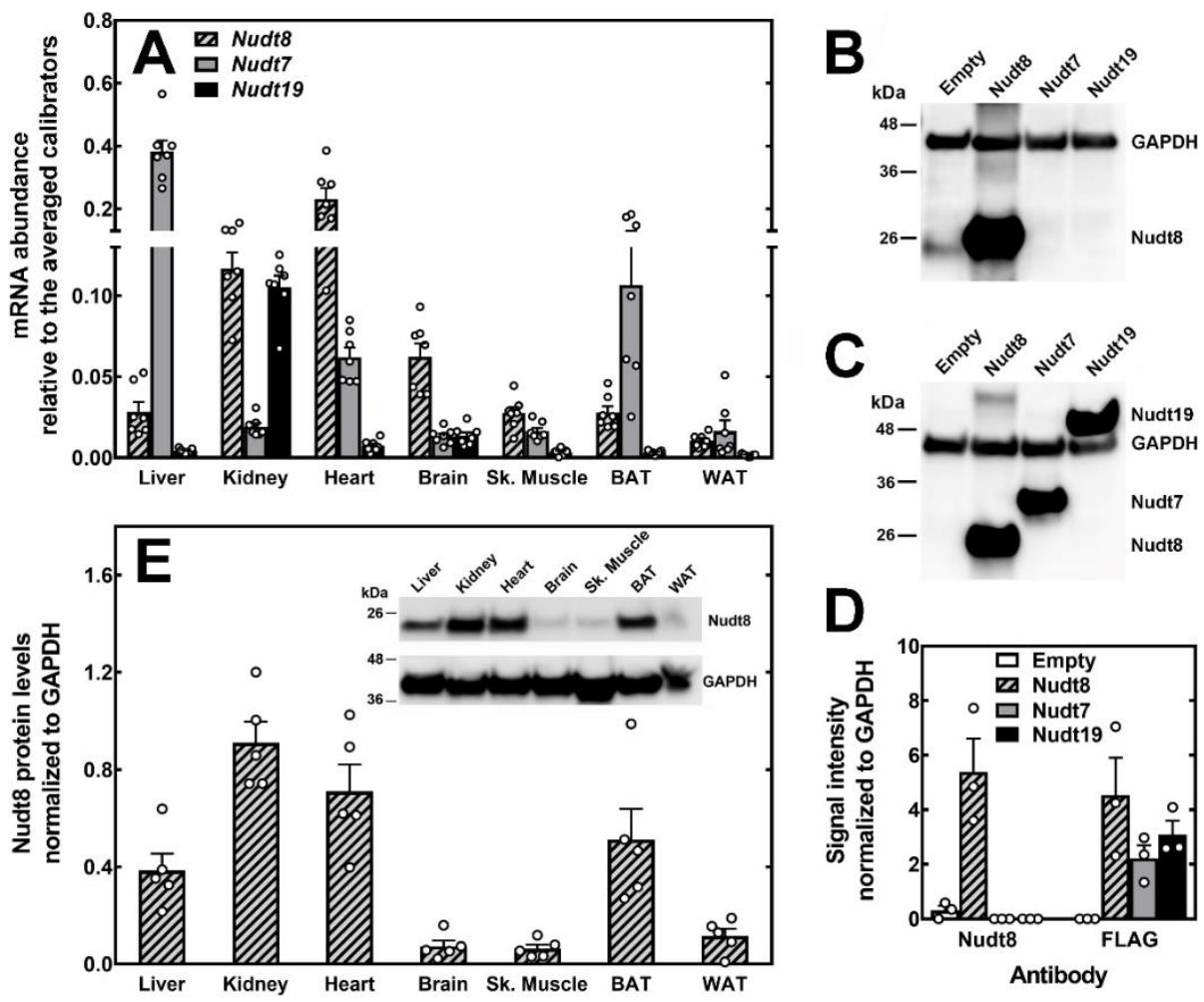

Figure 2.3. Relative abundance and tissue distribution of Nudt8 mRNA and protein. (A) Relative abundance of mouse Nudt8, Nudt7, and Nudt19 transcripts in selected tissues. Data are reported as the mean (bars) of measurements on individual mice (circles) \pm SEM. (B-D) HEK293T cells were transfected with either an empty plasmid or plasmids carrying the coding sequences for the FLAG-tagged versions of Nudt8, Nudt7, and Nudt19. Representative western blots showing that (B) the Nudt8 antibody did not cross-react with Nudt7 or Nudt19, while (C) the FLAG antibody detected all isoforms. (D) Quantification of the Nudt8 and FLAG signals from 3 independent transfections (circles) \pm SEM. (E) Levels of Nudt8 protein in mouse tissues as quantified by western blot analysis. Data are reported as the mean (bars) of measurements of the Nudt8 signal in tissues from individual mice (circles) \pm SEM. Inset, representative western blot. Abbreviations: Sk. Muscle or Sk. M., skeletal muscle; BAT, brown adipose tissue; WAT, white adipose tissue. GAPDH was used to normalize the Nudt8 and FLAG signals in (B) and (C) and to normalize the tissue Nudt8 signal in (E).

\section{Nudt8 exhibits a broad tissue distribution and its expression does not change with the nutritional state}

Nudt7 and Nudt19 exhibit a very narrow tissue distribution, predominating in the liver and kidneys, respectively $(13,15,16)$. To determine the tissue distribution and relative transcript abundance of Nudt8 with respect to Nudt7 and Nudt19, we measured the mRNA levels of all three 
enzymes in different organs. We found that Nudt8 was the major isoform in the heart, brain, and skeletal muscle. Furthermore, Nudt8 mRNA in the kidneys was as abundant as Nudt19 mRNA (Fig. 2.3A). We next generated a polyclonal antibody to measure Nudt8 protein expression in each tissue. To determine the isoform specificity of the Nudt8 antibody, we analyzed lysates from HEK293T cells transfected with constructs encoding FLAG-tagged versions of Nudt8, Nudt7, and Nudt19, or the empty vector. While the FLAG antibody detected all three enzymes (Fig. 2.3C), the Nudt8 antibody recognized only Nudt8 (Fig. 2.3B), indicating isoform specificity. Western blot
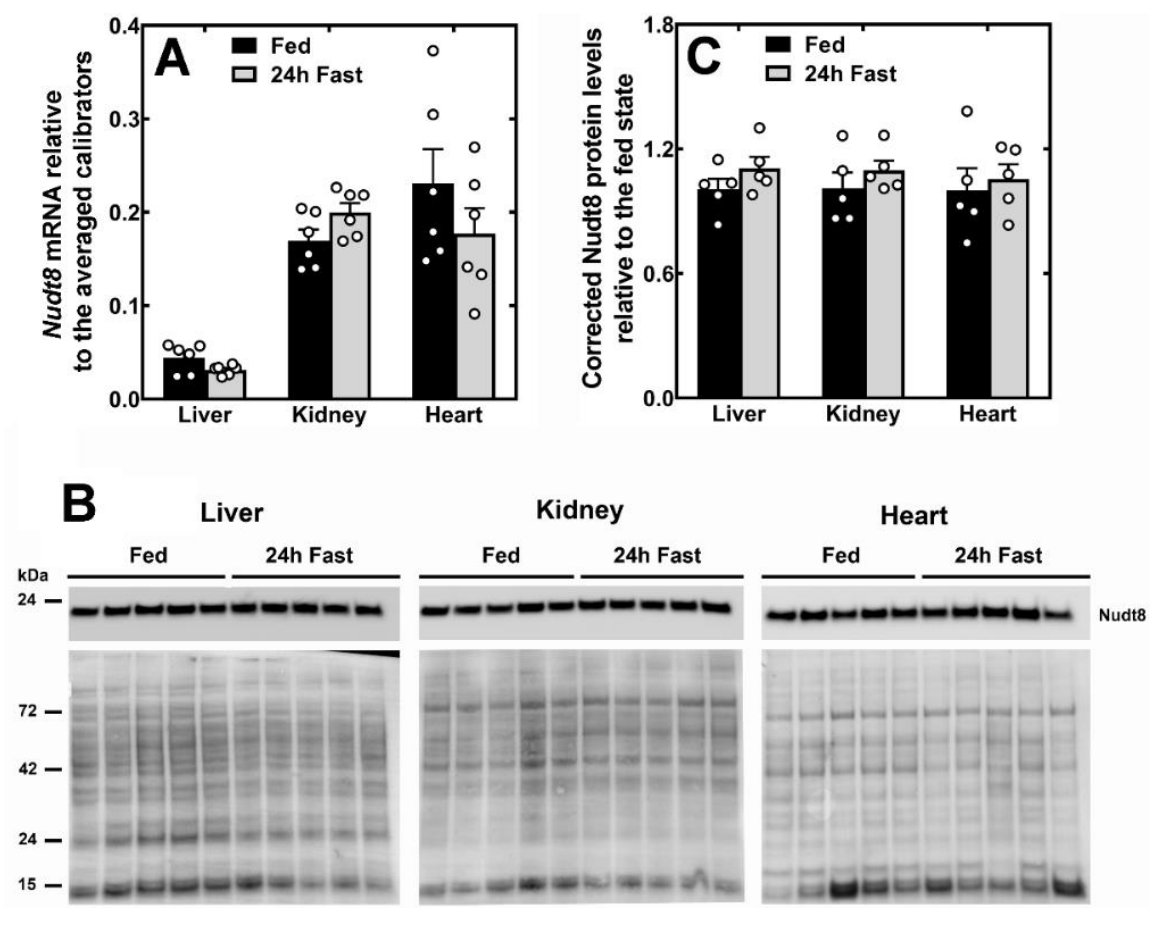

Figure 2.4. Nudt8 expression in the liver, kidneys and heart of fed and fasted mice. (A) Nudt8 mRNA in the liver, kidneys and heart of mice fed ad libitum or fasted for 24 h. (B) Western blot analysis showing that Nudt8 protein levels do not change with the nutritional state. Total protein loading is shown under each immunoblot. (C) Quantification of the western blots in (B) normalizing to total protein loading and expressing the corrected intensity relative to the fed state. Data in (A) and $(C)$ are shown as the mean (bars) of measurements on individual mice (circles) \pm SEM.

analysis of different tissues showed that Nudt8 was expressed at the highest levels in the kidneys, heart, brown adipose tissue, and liver (Fig. 2.3E). Detectable, but significantly lower levels of the 
enzyme were also found in the brain, skeletal muscle, and white adipose tissue. Combined, these data indicated that Nudt8 had a much broader tissue distribution than Nudt7 and Nudt19.

Nudt7 is regulated by the nutritional state and its mRNA and protein levels in the liver decrease with fasting $(16,19)$. Conversely, the expression of Nudt19 in the kidneys remains constant between the fed and fasted states, despite the contribution of Nudt19 to the regulation of the concentration of $\mathrm{CoA}$ in the fed state (13). We quantified Nudt8 mRNA and protein levels in the liver, kidneys and heart of mice fed ad libitum or fasted for $24 \mathrm{~h}$. Similar to Nudt19, but unlike the closely related Nudt7, Nudt8 expression did not change with the nutritional state in any of the tissue analyzed (Fig. 2.4), suggesting that regulation might occur through a different mechanism.

\section{Mitochondrial localization of Nudt8}

Many eukaryotic CoA diphosphohydrolases, including Nudt7 and Nudt19, localize to the peroxisomes. Nudt8 does not contain a peroxisomal targeting signal; instead, its $\mathrm{N}$-terminus contains a putative mitochondrial targeting sequence within the first 24 residues, as predicted by both TargetP (31) and MitoFates (32). Furthermore, Nudt8 is annotated as a mitochondrial protein in the Mitocarta 2.0 database, an inventory of mouse and human proteins whose mitochondrial localization is supported by a combination of computational and proteomics data (27). To verify the subcellular localization of Nudt8 in intact cells, HEK293 cells were transiently transfected with a construct expressing Nudt8 with a C-terminal FLAG tag. Using the FLAG tag antibody, we found that Nudt8 co-localized with the mitochondrial marker MitoTracker Orange CMTMRos, but not with the peroxisomal marker PMP70 (Fig. 2.5A-F). To corroborate this conclusion in mouse tissue, we fractionated liver homogenates with a protocol that achieved some degree of separation between peroxisomes and mitochondria (Fig. 2.5G-H). While endogenous 

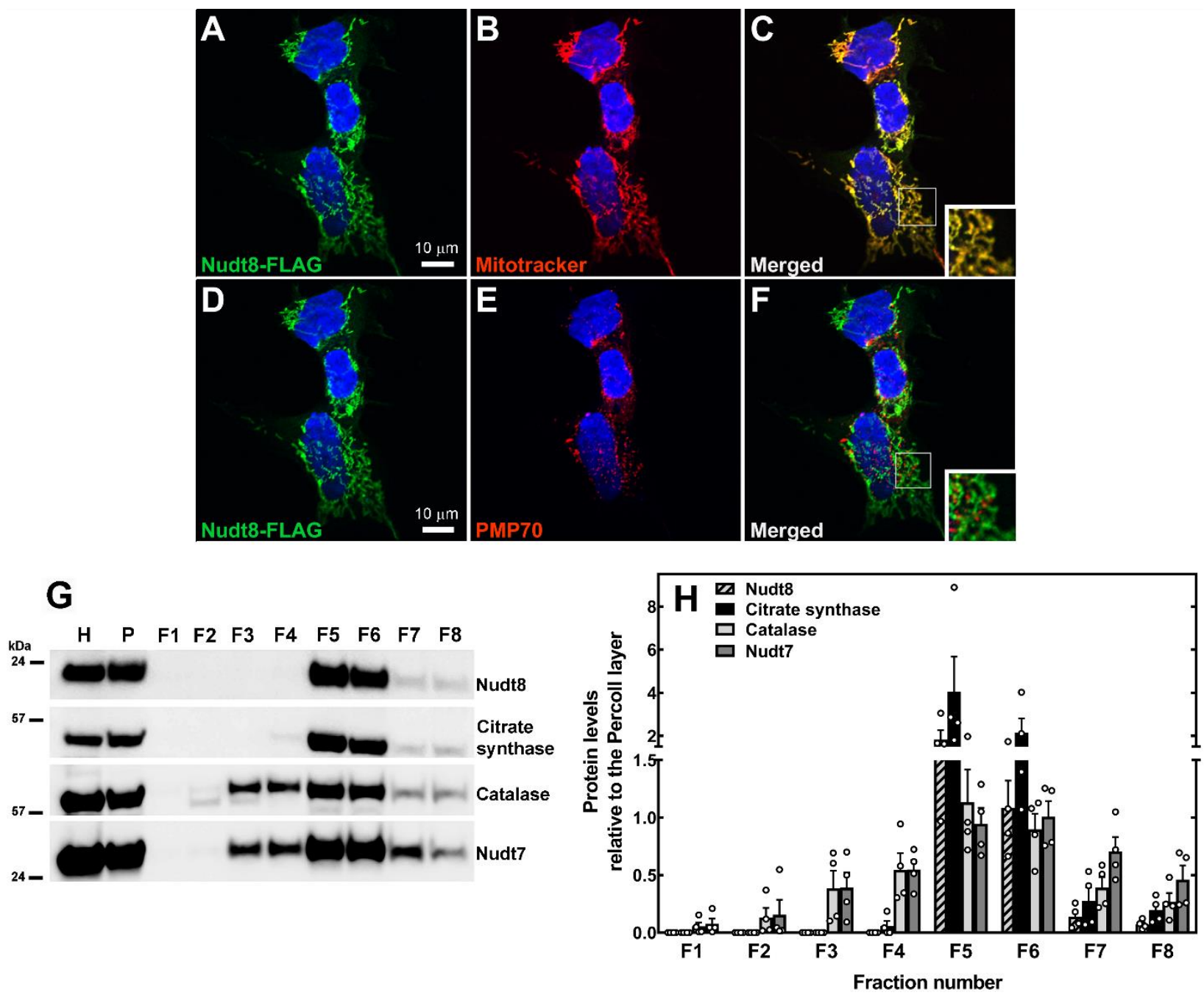

Figure 2.5. Mitochondrial localization of Nudt8. HEK293 cells were transfected with plasmids encoding Nudt8 with a C-terminal FLAG tag, fixed, and analyzed by confocal microscopy. (A, C, D, F) Nudt8, visualized with the FLAG antibody, is in green. (B-C) Mitochondria, visualized using Mitotracker Orange CMTMRos, are in red. (E-F) Endogenous PMP70, used as a peroxisomal marker, is in red. Cell nuclei were stained with DAPI, in blue. Insets in merged images show details at higher magnification. The results are representative of at least 2 independent experiments. $(\mathrm{G}-\mathrm{H})$ Subcellular fractionation of mouse livers. Nudt8 exhibited a sedimentation profile similar to the mitochondrial marker citrate synthase. Catalase and Nudt7 were used as peroxisomal markers. The results are representative of 4 independent experiments quantified in $(\mathrm{H})$ relative to the input in the Percoll layer, $\mathrm{P} . \mathrm{H}$, liver homogenate; F1-8, fractions 1-8.

Nudt8 was found in mitochondria-enriched fractions (Fig. 2.5G-H, fractions F5-F6), exhibiting the same fractionation profile as the mitochondrial marker citrate synthase, this enzyme was not detected in fractions containing purified peroxisomes (Fig. 2.5G-H, fractions F2-F4), as marked 
by both catalase and Nudt7. Combined, these results supported the conclusion that Nudt8 localized exclusively to the mitochondria.

\section{Discussion}

Until now, only liver and kidney peroxisomes were known to contain enzymes capable of degrading CoA $(14,15)$. The major finding of this work is that Nudt8 is a novel CoA diphosphohydrolase that localizes to the mitochondria and has a broad tissue distribution. Thus, our data strongly suggest that regulation of CoA levels by degradation occurs in more organs than previously thought and in both peroxisomes and mitochondria.

Recombinant mouse Nudt8 readily hydrolyzed free CoA and a variety of short-chain acylCoAs but exhibited a relatively low activity against acetyl-CoA (Fig. 2.2C and Table 2.1). Additionally, this enzyme showed a distinctive preference for $\mathrm{Mn}^{2+}$ ions that sets it apart from the peroxisomal isoforms Nudt7 and Nudt19, which can use $\mathrm{Mg}^{2+}$ or $\mathrm{Mn}^{2+}$ (Fig. 2.2B) $(14,15)$. Given some of the properties of $\mathrm{Mn}^{2+}$, this strong metal preference could potentially play a role in the substrate specificity of Nudt8 and, in particular, in the low binding affinity that the enzyme showed towards acetyl-CoA compared to the closely related Nudt7 (16). Indeed, $\mathrm{Mn}^{2+}-$ ligand bonds are more flexible than the equivalent $\mathrm{Mg}^{2+}$-ligand bonds both in length and angle. Furthermore, $\mathrm{Mn2+}$ binds nitrogen-containing ligands (e.g. histidines) more frequently than $\mathrm{Mg}^{2+}$ and can form bidentate complexes with aspartate and glutamate residues $(33,34)$. In the case of Nudix hydrolases the latter property may be particularly relevant given that the Nudix box motif

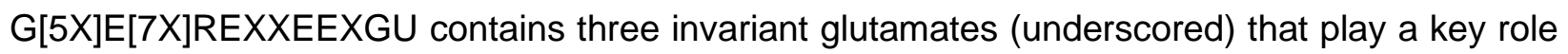
in the coordination of the catalytically essential metal ion. While this motif is highly conserved between Nudt7 and Nudt8, the binding of $\mathrm{Mn}^{2+}$ may impart a unique geometry to the Nudt8 active site and affect substrate binding. Crystal structures of Nudt7 and Nudt8 with substrate and preferred metal bound will be required to determine whether the presence of $\mathrm{Mn}^{2+}$ can explain how Nudt8 better discriminates between acetyl-CoA and other CoA substrates compared to 
Nudt7. This substrate specificity, in turn, may be relevant to the physiological function of Nudt8. Under our assay conditions, the optimal $\mathrm{MnCl}_{2}$ concentration for Nudt8 was $2 \mathrm{mM}$, which is within the physiological range of the estimated $\mathrm{Mn}^{2+}$ concentration inside the mitochondria $(35,36)$. Interestingly, significant activation by $\mathrm{Mn}^{2+}$ has also been observed for other mitochondrial Nudix hydrolases including mouse Nudt13, an enzyme that degrades $\operatorname{NADP}(\mathrm{H})$, and AtNudt15, a CoA diphosphohydrolase in A. thaliana that, however, was not tested against acetyl-CoA $(37,38)$. Importantly, the existence of Nudt8 and its CoA-degrading activity may explain how, even in the absence of Nudt19 and any detectable levels of Nudt7, the concentration of CoA could still be significantly decreased in the kidneys of $\mathrm{Nudt}_{19^{-/}}$mice during the transition from the fasted to the fed state (13).

Nudt8 was highly expressed in all the mitochondria-rich organs analyzed except the skeletal muscle (Fig. 2.3A). This is not unusual, as different organs contain variable levels of mitochondrial proteins depending on the functional emphasis of their mitochondria $(39,40)$. Interestingly, the expression of Nudt8 closely correlated with the concentration of CoA in those organs (41), and the expression of Nudt8 in skeletal muscle, which contain a relatively small CoA pool, may be proportionally adjusted to the concentration of the cofactor to avoid depleting it. At the subcellular level, mitochondria contain the highest concentration of cellular $\operatorname{CoA}(7,8)$ and are the site of several CoA-dependent metabolic processes, including glucose and fatty acid oxidation. CoA is present in both the mitochondrial matrix and the intermembrane space. While the localization of Nudt8 places it in a position to modulate mitochondrial metabolism, the precise physiological role of this enzyme will depend on the submitochondrial CoA pool that it can actually access. Using independent spatially restricted enzyme tagging-based approaches, two studies have recently identified human NUDT8 as a resident of the inner membrane/matrix in HEK293T cells $(42,43)$, which suggests that Nudt8 may regulate the matrix CoA pool. Such localization would also potentially place Nudt8 in the same compartment as CoA synthase (Coasy), the last enzyme in the CoA biosynthetic pathway (44-46). Coasy catalyzes the formation of dephospho- 
CoA from ATP and phosphopantetheine and the final phosphorylation of dephospho-CoA to CoA. Coasy has been localized to both the outer mitochondrial membrane (46) and the inner membrane/matrix (42). In the absence of regulatory mechanisms, the latter subcellular localization would allow Coasy to re-synthesize CoA from the phosphopantetheine produced by Nudt8, generating wasteful cycles of CoA synthesis and degradation. Our data indicate that in the kidneys, heart and liver, organs with high expression of Nudt8 and detectable CoA-degrading activity $(13,47,48)$, Nudt8 levels did not change with the nutritional state. Additional studies will be required to determine whether other potential regulatory mechanisms, including posttranslational modifications or regulation by metabolites in the mitochondria, may control the activity of Nudt8 and whether Coasy and Nudt8 actually localize to the same sub-mitochondrial compartment.

In conclusion, the identification and characterization of Nudt8 as a mitochondrial CoAdegrading enzyme indicates that CoA degradation is not limited to the peroxisomes and may provide a common mechanism to regulate the subcellular pools of CoA in response to changes in the metabolic state.

Acknowledgements: We thank Deborah Corbin, Mason Colbert, Dominique Saporito, Paolo Fagone in the Biochemistry Protein Core Facility, and the Imaging Facilities at West Virginia University for their expert technical assistance. This work was supported by West Virginia University's School of Medicine startup foundation funding (RL), and National Institutes of Health Grants R35GM119528 (RL), F31DK118878 (EWK), and F31GM126838 (SAS). The West Virginia University Imaging Facilities were supported by the West Virginia University Cancer Institute and NIH grants P20RR016440, P30RR032138, P30GM103488 and U54GM104942. 


\section{References}

1. Choudhary, C., Weinert, B. T., Nishida, Y., Verdin, E., and Mann, M. (2014) The growing landscape of lysine acetylation links metabolism and cell signalling. Nature reviews. Molecular cell biology 15, 536-550

2. Hirschey, M. D., and Zhao, Y. (2015) Metabolic Regulation by Lysine Malonylation, Succinylation, and Glutarylation. Molecular \& cellular proteomics : MCP 14, 2308-2315

3. Resh, M. D. (2016) Fatty acylation of proteins: The long and the short of it. Progress in lipid research 63, 120-131

4. Daniotti, J. L., Pedro, M. P., and Valdez Taubas, J. (2017) The role of S-acylation in protein trafficking. Traffic

5. Sabari, B. R., Zhang, D., Allis, C. D., and Zhao, Y. (2017) Metabolic regulation of gene expression through histone acylations. Nature reviews. Molecular cell biology 18, 90-101

6. Gout, I. (2018) Coenzyme A, protein CoAlation and redox regulation in mammalian cells. Biochem Soc Trans 46, 721-728

7. Horie, S., Ishii, H., and Suga, T. (1981) Changes in peroxisomal fatty acid oxidation in the diabetic rat liver. J Biochem 90, 1691-1696

8. Van Broekhoven, A., Peeters, M. C., Debeer, L. J., and Mannaerts, G. P. (1981) Subcellular distribution of coenzyme A: evidence for a separate coenzyme A pool in peroxisomes. Biochemical and biophysical research communications 100, 305-312

9. Peng, Y., and Puglielli, L. (2016) N-lysine acetylation in the lumen of the endoplasmic reticulum: A way to regulate autophagy and maintain protein homeostasis in the secretory pathway. Autophagy 12, 1051-1052

10. Idell-Wenger, J. A., Grotyohann, L. W., and Neely, J. R. (1978) Coenzyme A and carnitine distribution in normal and ischemic hearts. The Journal of biological chemistry 253, 43104318

11. Leonardi, R., Rehg, J. E., Rock, C. O., and Jackowski, S. (2010) Pantothenate kinase 1 is required to support the metabolic transition from the fed to the fasted state. PLoS One 5, e11107

12. Shumar, S. A., Kerr, E. W., Fagone, P., Infante, A. M., and Leonardi, R. (2019) Overexpression of Nudt7 decreases bile acid levels and peroxisomal fatty acid oxidation in the liver. Journal of lipid research

13. Shumar, S. A., Kerr, E. W., Geldenhuys, W. J., Montgomery, G. E., Fagone, P., Thirawatananond, P., Saavedra, H., Gabelli, S. B., and Leonardi, R. (2018) Nudt19 is a renal CoA diphosphohydrolase with biochemical and regulatory properties that are distinct from the hepatic Nudt7 isoform. J Biol Chem 293, 4134-4148

14. Gasmi, L., and McLennan, A. G. (2001) The mouse Nudt7 gene encodes a peroxisomal nudix hydrolase specific for coenzyme A and its derivatives. The Biochemical journa/357, 33-38

15. Ofman, R., Speijer, D., Leen, R., and Wanders, R. J. (2006) Proteomic analysis of mouse kidney peroxisomes: identification of RP2p as a peroxisomal nudix hydrolase with acylCoA diphosphatase activity. The Biochemical journal 393, 537-543

16. Reilly, S. J., Tillander, V., Ofman, R., Alexson, S. E., and Hunt, M. C. (2008) The nudix hydrolase 7 is an Acyl-CoA diphosphatase involved in regulating peroxisomal coenzyme A homeostasis. Journal of biochemistry 144, 655-663

17. Antonenkov, V. D., and Hiltunen, J. K. (2006) Peroxisomal membrane permeability and solute transfer. Biochimica et biophysica acta 1763, 1697-1706

18. Hunt, M. C., Tillander, V., and Alexson, S. E. (2014) Regulation of peroxisomal lipid metabolism: the role of acyl-CoA and coenzyme A metabolizing enzymes. Biochimie 98, 45-55 
19. Leonardi, R., Rock, C. O., and Jackowski, S. (2014) Pank1 deletion in leptin-deficient mice reduces hyperglycaemia and hyperinsulinaemia and modifies global metabolism without affecting insulin resistance. Diabetologia 57, 1466-1475

20. Bessman, M. J., Frick, D. N., and O'Handley, S. F. (1996) The MutT proteins or "Nudix" hydrolases, a family of versatile, widely distributed, "housecleaning" enzymes. The Journal of biological chemistry 271, 25059-25062

21. Carreras-Puigvert, J., Zitnik, M., Jemth, A. S., Carter, M., Unterlass, J. E., Hallstrom, B., Loseva, O., Karem, Z., Calderon-Montano, J. M., Lindskog, C., Edqvist, P. H., Matuszewski, D. J., Ait Blal, H., Berntsson, R. P. A., Haggblad, M., Martens, U., Studham, M., Lundgren, B., Wahlby, C., Sonnhammer, E. L. L., Lundberg, E., Stenmark, P., Zupan, B., and Helleday, T. (2017) A comprehensive structural, biochemical and biological profiling of the human NUDIX hydrolase family. Nature communications 8, 1541

22. McLennan, A. G. (2006) The Nudix hydrolase superfamily. Cellular and molecular life sciences : CMLS 63, 123-143

23. de la Pena, A. H., Suarez, A., Duong-Ly, K. C., Schoeffield, A. J., Pizarro-Dupuy, M. A., Zarr, M., Pineiro, S. A., Amzel, L. M., and Gabelli, S. B. (2015) Structural and Enzymatic Characterization of a Nucleoside Diphosphate Sugar Hydrolase from Bdellovibrio bacteriovorus. PloS one 10, e0141716

24. Cartwright, J. L., Gasmi, L., Spiller, D. G., and McLennan, A. G. (2000) The Saccharomyces cerevisiae PCD1 gene encodes a peroxisomal nudix hydrolase active toward coenzyme A and its derivatives. The Journal of biological chemistry 275, 3292532930

25. AbdelRaheim, S. R., and McLennan, A. G. (2002) The Caenorhabditis elegans Y87G2A.14 Nudix hydrolase is a peroxisomal coenzyme A diphosphatase. BMC biochemistry 3, 5

26. Ito, D., Yoshimura, K., Ishikawa, K., Ogawa, T., Maruta, T., and Shigeoka, S. (2012) A comparative analysis of the molecular characteristics of the Arabidopsis CoA pyrophosphohydrolases AtNUDX11, 15, and 15a. Bioscience, biotechnology, and biochemistry 76, 139-147

27. Calvo, S. E., Clauser, K. R., and Mootha, V. K. (2016) MitoCarta2.0: an updated inventory of mammalian mitochondrial proteins. Nucleic acids research 44, D1251-1257

28. Studier, F. W. (2005) Protein production by auto-induction in high density shaking cultures. Protein Expr Purif 41, 207-234

29. Antonenkov, V. D., Sormunen, R. T., and Hiltunen, J. K. (2004) The behavior of peroxisomes in vitro: mammalian peroxisomes are osmotically sensitive particles. American journal of physiology. Cell physiology 287, C1623-1635

30. Garcia, M., Leonardi, R., Zhang, Y. M., Rehg, J. E., and Jackowski, S. (2012) Germline deletion of pantothenate kinases 1 and 2 reveals the key roles for CoA in postnatal metabolism. PloS one 7, e40871

31. Emanuelsson, O., Brunak, S., von Heijne, G., and Nielsen, H. (2007) Locating proteins in the cell using TargetP, SignalP and related tools. Nature protocols 2, 953-971

32. Fukasawa, Y., Tsuji, J., Fu, S. C., Tomii, K., Horton, P., and Imai, K. (2015) MitoFates: improved prediction of mitochondrial targeting sequences and their cleavage sites. Molecular \& cellular proteomics : MCP 14, 1113-1126

33. Bock, C. W., Katz, A. K., Markham, G. D., and Glusker, J. P. (1999) Manganese as a Replacement for Magnesium and Zinc: Functional Comparison of the Divalent Ions. Journal of the American Chemical Society 121, 7360-7372

34. Kehres, D. G., and Maguire, M. E. (2003) Emerging themes in manganese transport, biochemistry and pathogenesis in bacteria. FEMS Microbiol Rev 27, 263-290 
35. Gunter, T. E., Miller, L. M., Gavin, C. E., Eliseev, R., Salter, J., Buntinas, L., Alexandrov, A., Hammond, S., and Gunter, K. K. (2004) Determination of the oxidation states of manganese in brain, liver, and heart mitochondria. Journal of neurochemistry 88, 266-280

36. Gunter, R. E., Puskin, J. S., and Russell, P. R. (1975) Quantitative magnetic resonance studies of manganese uptake by mitochondria. Biophys $J 15,319-333$

37. Abdelraheim, S. R., Spiller, D. G., and McLennan, A. G. (2017) Mouse Nudt13 is a Mitochondrial Nudix Hydrolase with NAD(P)H Pyrophosphohydrolase Activity. The protein journal 36, 425-432

38. Ogawa, T., Yoshimura, K., Miyake, H., Ishikawa, K., Ito, D., Tanabe, N., and Shigeoka, S. (2008) Molecular characterization of organelle-type Nudix hydrolases in Arabidopsis. Plant physiology 148, 1412-1424

39. Forner, F., Foster, L. J., Campanaro, S., Valle, G., and Mann, M. (2006) Quantitative proteomic comparison of rat mitochondria from muscle, heart, and liver. Molecular \& cellular proteomics : MCP 5, 608-619

40. Johnson, D. T., Harris, R. A., French, S., Blair, P. V., You, J., Bemis, K. G., Wang, M., and Balaban, R. S. (2007) Tissue heterogeneity of the mammalian mitochondrial proteome. American journal of physiology. Cell physiology 292, C689-697

41. Dansie, L. E., Reeves, S., Miller, K., Zano, S. P., Frank, M., Pate, C., Wang, J., and Jackowski, S. (2014) Physiological roles of the pantothenate kinases. Biochemical Society transactions 42, 1033-1036

42. Rhee, H. W., Zou, P., Udeshi, N. D., Martell, J. D., Mootha, V. K., Carr, S. A., and Ting, A. Y. (2013) Proteomic mapping of mitochondria in living cells via spatially restricted enzymatic tagging. Science (New York, N.Y.) 339, 1328-1331

43. Liu, X., Salokas, K., Tamene, F., Jiu, Y., Weldatsadik, R. G., Ohman, T., and Varjosalo, M. (2018) An AP-MS- and BiolD-compatible MAC-tag enables comprehensive mapping of protein interactions and subcellular localizations. Nature communications 9, 1188

44. Aghajanian, S., and Worrall, D. M. (2002) Identification and characterization of the gene encoding the human phosphopantetheine adenylyltransferase and dephospho-CoA kinase bifunctional enzyme (CoA synthase). The Biochemical journal 365, 13-18

45. Daugherty, M., Polanuyer, B., Farrell, M., Scholle, M., Lykidis, A., de Crecy-Lagard, V., and Osterman, A. (2002) Complete reconstitution of the human coenzyme A biosynthetic pathway via comparative genomics. The Journal of biological chemistry 277, 21431-21439

46. Zhyvoloup, A., Nemazanyy, I., Panasyuk, G., Valovka, T., Fenton, T., Rebholz, H., Wang, M. L., Foxon, R., Lyzogubov, V., Usenko, V., Kyyamova, R., Gorbenko, O., Matsuka, G., Filonenko, V., and Gout, I. T. (2003) Subcellular localization and regulation of coenzyme A synthase. J Biol Chem 278, 50316-50321

47. Lopaschuk, G. D., and Neely, J. R. (1987) Coenzyme A degradation in the heart: effects of diabetes and insulin. Journal of molecular and cellular cardiology 19, 281-288

48. Lopaschuk, G. D., and Neely, J. R. (1987) Stimulation of myocardial coenzyme A degradation by fatty acids. The American journal of physiology 253, H41-46 


\section{Chapter III: Effect of liver specific overexpression of Nudt8 on mitochondrial metabolism}

\section{Abstract}

Coenzyme $\mathrm{A}(\mathrm{Co} A)$ is an essential cofactor that drives a multitude of metabolic reactions within the cell. There are three pools of CoA located in the mitochondria, peroxisomes, and cytosol that support metabolic processes specific to those organelles. In the fasted state, increased CoA biosynthesis drives fatty acid oxidation, gluconeogenesis and ketogenesis in the liver. Upon refeeding, CoA levels are rapidly decreased as a result of decreased synthesis and active degradation. Recently, Nudt8 was found to be a CoA-degrading enzyme that localizes to the mitochondria. In the liver, approximately $80 \%$ of (acyl-)CoA is in the mitochondrial matrix, thus Nudt8 is positioned to regulate the largest pool of (acyl-)CoAs in the cell. To gain insight into the role of Nudt8 in the regulation of the mitochondrial acyl-CoA pool, this protein was overexpressed using a liver-specific adeno-associated virus (AAV). An AAV carrying GFP was used as a control. Overexpression of Nudt8 did not affect total CoA levels in the mitochondria or cytosolic fractions. Furthermore, analysis of changes to individual CoA species by LC/MS/MS showed no major changes to the composition of the acyl-CoA pool. Despite a 1.5-fold increase in CoA-degrading activity in the livers of the Nudt8-AAV mice, this did not translate into alterations in CoA metabolism. One possible explanation is that CoA biosynthesis likely compensated for the increase in Nudt8-mediated degradation in order to maintain the mitochondrial CoA pool. 


\section{Introduction}

Coenzyme $\mathrm{A}(\mathrm{CoA})$ is an integral cofactor that forms a thioester bond with carboxylic acids to form acyl-CoA thioesters (1). Hydrolysis of this thioester bond is energetically favorable allowing CoA to act as an acyl-donor that drives a multitude of metabolic reactions including fatty acid synthesis and oxidation, ketone synthesis and oxidation, tricarboxylic acid cycle, and acetylcholine synthesis (2,3). Additionally, (acyl-)CoAs can act as acyl-donors to posttranslationally modify proteins, effecting gene expression, protein stability and subcellular localization (4-6). Through allosteric regulation, CoA and (acyl-)CoAs can directly regulate flux through metabolic pathways (3). Recently, it was shown that in conditions of metabolic stress, the thiol of CoA can directly react with cysteines of target proteins resulting in 'CoAlation' $(7,8)$.

CoA levels are tightly regulated through cycles of synthesis and degradation (9). In the fasted state, accumulation of long-chain acyl-carnitines activate CoA biosynthesis through the allosteric activation of the initial and rate-limiting enzyme Pantothenate kinase (PanK) (10) . The increase in free CoA that occurs in the fasted state directs cellular metabolism towards processes like fatty acid $\beta$-oxidation, ketogenesis and increased flux through the gluconeogenic pathway in the liver (10). Upon refeeding, a rapid decrease in CoA levels shifts metabolism away from fatty acid oxidation and promotes glucose oxidation and lipogenesis $(10,11)$. This net decrease in CoA levels occurs through inhibition of biosynthesis and active degradation (12). The importance of this tight regulation of $\mathrm{CoA}$ levels is underscored as prolonged elevations in total CoA through transgenic expression of PANK2 resulted in decreased ATP production, decreased Complex I activity, and increased reactive oxygen species (13). Further, the Lep $^{-/}$mouse model was found to have increased total hepatic CoA levels that drive fatty acid oxidation and gluconeogenesis that contributes to the hyperglycemic phenotype (11). Importantly, reduction in (acyl-)CoA levels corrects the diabetic phenotype independent of insulin signaling (11). 
To date, the only known CoA-degrading enzymes belong to the Nudix hydrolase superfamily. CoA-degrading Nudix enzymes hydrolyze (acyl-)CoAs creating 3',5'-ADP and an (acyl-)phosphopantetheine (9). Nudt7 and Nudt19 are peroxisomal CoA-degrading enzymes that are expressed in the liver and kidneys respectively (14-17). While we don't have a complete understanding of the role of Nudt7 and Nudt19 on CoA regulation, it is likely confined to the peroxisomal CoA pool, as deletion of these proteins does not prevent the decrease in total CoA that is associated with the transition from fasted to fed nutritional states (16). Recently, Nudt8 was identified as a novel CoA-degrading enzyme that has a wide tissue distribution and localizes to the mitochondria (18). In the liver, the mitochondria contain approximately $80 \%$ of the cellular (acyl-)CoAs, thus the broad tissue distribution of Nudt8 coupled with its prescence in the mitochondria indicate it could be an important regulator of (acyl-)CoA (9).

The mitochondrial matrix contains of the highest concentration of CoA within the cell. The mitochondrial inner membrane is impermeable to CoA, which is thought to be imported into the matrix by Solute carrier proteins; however, this has not been confirmed in vivo (19-21). To gain insight into the role of Nudt8 and its ability to regulate the mitochondrial (acyl-)CoA pool, we first determined its submitochondrial localization. Using a protease protection assay in conjunction with proximity tagging we were able to show that Nudt8 localizes to the mitochondrial matrix. To gain insight into in vivo substrates of Nudt8 we overexpressed it in the liver of C57BI/6J mice using a liver-specific AAV mediated expression. Despite robust overexpression of the transgene, Nudt8 had no effect on the total CoA levels in the mitochondria. Additionally, we examined changes on free CoA and individual acyl-CoA species via mass spectrometry and found that the transgene did not have a significant effect on the composition of the acyl-CoA pool, as only a few acyl-CoA species were significantly altered. Taken together, our data indicates that despite increase in (acyl-)CoA degradation in the liver, CoA biosynthesis likely compensated for this effect. 


\section{Materials and Methods}

Reagents were purchased from the following suppliers: oligonucleotides, Lipofectamine 2000, cell culture reagents, HRP-conjugated goat anti-rabbit lgG, MitoTracker Red CMXRos, and HRPStreptavidin from ThermoFisher Scientific; restriction enzymes from New England Biolabs; NIH3T3 cells from the American Type Culture Collection. The Mito-V5-APEX plasmid was obtained from Addgene (\#72480). The Dylight 488 conjugated goat anti- mouse secondary from Rockland Antibodies and Assays. The antibodies against the FLAG epitope and alpha tubulin were obtained from Sigma-Aldrich. The citrate synthase and cytochrome $\mathrm{C}$ antibodies from Abcam. The antibody against the full-length mouse Nudt8 was generated as previously described (18). mBB-CoA was synthesized as previously described (22). The 3',5'-ADP used as standard for the product of the Nudt8 reaction was purchased from Sigma-Aldrich. All other reagents were of analytical grade or better and were purchased from Sigma-Aldrich or Fisher Scientific, unless otherwise stated.

\section{Adeno-Associated Virus production}

Plasmid pscAAV-LP1, carrying the green fluorescent protein coding sequence (GFP), was developed by John T. Gray and provided by St. Jude's Children's Hospital, Memphis, TN. Generation of the psc-AAV-LP1-mNudt8 was performed as follows: Nudt8 in pET28A was excised with Nde1 and Not1 and ligated it into the psc-AAV-LP1. AAV was produced as described in (16).

\section{Animal Studies}

Six week- old C57BL/6J male mice were purchased from Jackson Laboratory. Mice were fed a standard chow diet (Tekland 2018S) and were housed in a room that was maintained at a standard temperature of $72.0^{\circ} \mathrm{F}, 40 \% \pm 2 \%$. Animals were exposed to 12 -hour light/ dark cycles, with the dark cycle beginning at 18:00 h. Following a 2-week acclimation period, mice were injected, via tail vein, with $2.5{ }^{*} 10^{11}$ genome copies of the GFP- or Nudt8-AAV in PBS (150 $\mu$ l). Fasting experiments were started at 7:00 am, with mice being removed from cages and placed into cages with grids without food for 24-hours, unless otherwise noted. Blood glucose 
measurements were obtained via tail bleeding using a glucometer. Both fed and 24 hour fasted measurements were done at 7:00 am. Measurement of $\beta$ - hydroxybutyrate was performed on serum obtained from cardiac puncture and stored at $-80^{\circ} \mathrm{C}$ prior to analysis. Serum was analyzed using colorimetric assay kit from Cayman Chemical (700190).

\section{Immunofluorescence}

Confocal microscopy was utilized to confirm the mitochondrial localization of the Nudt8 transgene using primary hepatocytes isolated from mice injected with Nudt8-AAV. Hepatocytes were isolated as described previously (23). Prior to fixation with $4 \%$ paraformaldehyde, hepatocytes were incubated with $25 \mathrm{nM}$ MitoTracker Red CMXRos for 30 minutes at $37{ }^{\circ} \mathrm{C}$. Following fixation, hepatocytes were blocked in PBS containing 5\% FBS and $0.3 \%$ Triton-X-100 for 1 hour at room temperature. The FLAG antibody was used at a 1:1000 dilution in 5\% BSA solution prepared in PBS. Following an overnight incubation at $4^{\circ} \mathrm{C}$, hepatocytes were washed 10 times with PBS. Following washes, cells were incubated with Alexa Flour 488 secondary antibody for 1 hour at room temperature. Alexa Flour was used at a 1:500 dilution, in 1\% BSA, 0.3\% Triton-X-100. Slides were mounted using Prolong Gold Antifade with DAPI (Thermo Fisher). Imaging experiments were performed at the West Virginia University Imaging Facility using a Nikon A 1 R/N SIM- E confocal microscope. Image analysis and figures were prepared using ImageJ.

\section{Tissue Enzymatic Assays}

To measure total CoA diphosphohydrolase activity in liver homogenates, $20 \mathrm{mg}$ of frozen GFP- or Nudt8-AAV liver were homogenized in $750 \mu \mathrm{L}$ of $20 \mathrm{mM}$ Tris- $\mathrm{HCl}$, pH 8.5. Tissue homogenates were incubated on ice for 10 minutes and then centrifuged at 20,000 xg for 10 minutes. The supernatant was removed and increasing amounts of protein $(50-200 \mu \mathrm{g})$ were incubated with $100 \mu \mathrm{M}$ mBB-CoA for 10 minutes at $37^{\circ} \mathrm{C}$. Reactions were stopped by the addition 
of $20 \mu \mathrm{L}$ of $20 \%$ perchloric acid, neutralized with $22 \mu \mathrm{L}$ of $2 \mathrm{M}$ potassium carbonate, and incubated on ice for 10 minutes. Reactions were diluted 5-fold with water and were analyzed via high pressure liquid chromatography as described previously (17). The amount of 3',5'-ADP produced as a result of Nudix activity was determined using a standard curve of $3^{\prime}, 5^{\prime}$-ADP exposed to the same conditions as those of the reactions.

\section{Subcellular Fractionation}

Isolation of mitochondria was performed as described by Graham et al. (24). Briefly, liver was homogenized in liver homogenization medium (LHM; $200 \mathrm{mM}$ mannitol, $50 \mathrm{mM}$ sucrose, 10 $\mathrm{mM}$ potassium chloride, $1 \mathrm{mM}$ EDTA, $10 \mathrm{mM}$ HEPES, $\mathrm{pH}$ 7.4) via 5-6 strokes using a Teflon homogenizer. Lysates were centrifuged at $1,000 \mathrm{xg}$ for 10 minutes at $4^{\circ} \mathrm{C}$ in a swinging-bucket rotor. The supernatant was transferred to a $10 \mathrm{~mL}$ polycarbonate tube and centrifuged at 3,000 $x g$ using a $70.1 \mathrm{Ti}$ Beckman centrifuge rotor. The resulting supernatant was carefully removed and transferred to bell top tubes (Beckman \# 345829) and centrifuged at 100,000 $\mathrm{xg}$ for 1 hour. The resulting supernatant was removed and served as the cytosolic fraction.

The pellet from the 3,000 $\mathrm{xg}$ centrifugation was resuspended in LHM and was centrifuged at 3,000 $\mathrm{xg}$ for 10 minutes. This wash step was repeated twice. Following the final washing step, the resulting mitochondrial pellet was resuspended in $300 \mu \mathrm{l}$ of LHM and applied to $8 \mathrm{ml}$ of $30 \%$ percoll in LHM and centrifuged at 95,000 $\mathrm{xg}$ for 35 minutes in a 70.1 Ti Beckman centrifuge rotor. The mitochondrial layer was carefully removed and placed in a clean polycarbonate centrifuge tube, diluted in 4 volumes of LHM, and centrifuged at $6,300 \mathrm{xg}$. The resulting pellet was resuspended in $500 \mu \mathrm{l}$ of $\mathrm{LHM}, 400 \mu \mathrm{l}$ was used for analysis of total CoA and the remaining 100 $\mu l$ were utilized for protein determination.

Protease Protection Assay 
Protease protection assays were performed as described in Bekeova et. al (25). Briefly, freshly isolated mitochondria (see above) were resuspended in mitochondrial assay buffer (MAS; $70 \mathrm{mM}$ sucrose, $220 \mathrm{mM}$ mannitol, $10 \mathrm{mM}$ potassium phosphate, $5 \mathrm{mM}$ magnesium chloride, 2 $\mathrm{mM}$ HEPES, $0.2 \% \mathrm{BSA}, \mathrm{pH} 7.2$ ) to a final concentration of $25 \mathrm{mg} / \mathrm{ml}$. Each reaction totaled $40 \mu \mathrm{l}$ of MAS with $100 \mu \mathrm{g}$ of mitochondrial protein and $0.02 \mathrm{mg} / \mathrm{ml}$ proteinase $\mathrm{K}$, unless otherwise noted. Digitonin was added at the following final concentrations: $0,0.01 \%, 0.04 \%, 0.05 \%, 0.07 \%, 0.08 \%$, $0.09 \%, 0.1 \%$ and Triton-X-100 (0.1\%), which completely solubilizes the mitochondria, was used as a positive control. Reactions were incubated at $37^{\circ} \mathrm{C}$ for 15 minutes. Protease activity was stopped with the addition of PMSF to a final concentration of $3.25 \mathrm{mM} .10 \mu \mathrm{l}$ of each fraction were analyzed for Nudt8 (1:3000), Tim23 (Inner membrane marker; 1: 5000), and citrate synthase (matrix; 1:1000) via western blotting.

\section{Generation of NIH 3T3 Cells Stably Expressing Nudt8-FLAG}

The Nudt8-FLAG lentiviral expression construct was generated by PCR amplifying Nudt8-FLAG cDNA and inserting it into a pLenti6.3/V5-GW-LacZ (ThermoFisher Scientific) digested with Eco-RV by Gibson Assembly (New England Biolabs Inc). The integrity of the insert was confirmed by Sanger Sequencing (WVU Genomic CORE). Lentivirus were generated in 293FT cells using the ViraPower HiPerform Lentiviral Expression System (ThermoFisher Scientific) as per manufacturer's instructions. NIH 3T3 were infected with lentivirus and cells stably transduced were selected with Blasticidin. Clonal cell lines were screened for Nudt8FLAG by western blot analysis.

\section{APEX Proximity Biotinylation}

Apex-mediated biotinylation of proteins localizing to the mitochondrial matrix was performed as described in Hung et al. (26). Briefly, NIH 3T3 cells stably expressing Nudt8-FLAG were platted on to two $10 \mathrm{~cm}$ dishes and allowed to attach overnight and grow to approximately $70 \%$ confluence. Cells were transiently transfected using Lipofectamine 2000 with the APEX2 
plasmid, as per manufacturer's protocol. The medium was changed 24 hours following transfection, replaced with DMEM and incubated for another 24 hours. Following incubation, cells were treated with DMEM containing biotin-phenol (500 $\mu \mathrm{M}$ final concentration) for 30 minutes at $37^{\circ} \mathrm{C}$. $100 \mu \mathrm{l}$ of $100 \mathrm{mM} \mathrm{H} \mathrm{O}_{2}$ was added to the plate and cells were incubated at room temperature for 5 minutes. Cells were washed with $10 \mathrm{~mL}$ quenching solution $(10 \mathrm{mM}$ sodium ascorbate, $5 \mathrm{mM}$ Trolox, and $10 \mathrm{mM}$ sodium azide in PBS) three times at room temperature. Cells were collected via scraping and lysed in $200 \mu$ lysis buffer consisting of 20 mM HEPES, $100 \mathrm{mM}$ sodium chloride, $10 \%$ glycerol and $1 \%$ NP-40. Cell lysates were cleared via centrifugation at $20,000 x g$ for 10 minutes at $4^{\circ} \mathrm{C}$. Nudt8-FLAG was immunoprecipitated using Flag-M2 affinity gel, as instructed by the manufacturer. Antibodies against citrate synthase (matrix) and cytochrome $\mathrm{c}$ (IMS) were crosslinked to Pierce ${ }^{\mathrm{TM}} \mathrm{A} / \mathrm{G}$ Plus Agrose beads using disuccinimidyl suberate as instructed by the manufacturer. IP eluates were analyzed via western blotting. Elutions were split and fractionated in parallel gels. Following transfer, one membrane was probed with streptavidin$\operatorname{HRP}(1: 10,000)$ to assess biotinylation of the eluted protein, and the other membrane was used to assess the quality of immunoprecipitation.

\section{Total CoA and Acyl-CoA Pool Composition}

Total CoA (free CoA plus CoA thioesters) was quantified in livers of AAV-GFP and Nudt8AAV mice using alkaline hydrolysis and derivatization to monobromobimane as described previously (27). For quantification of total CoA in the mitochondrial and cytosolic fractions, $400 \mu \mathrm{L}$ of mitochondrial or cytosolic fractions were added to $1.6 \mathrm{ml}$ of $1 \mathrm{mM}$ potassium hydroxide and were incubated at $55^{\circ} \mathrm{C}$ for 2 hours to hydrolyze all acyl-CoAs to free CoA. Following incubation, the $\mathrm{pH}$ of the samples was adjusted 8 by addition of $160 \mu \mathrm{L} 1 \mathrm{M}$ Trizma- $\mathrm{HCl}$. mBB was added (1 $\mu \mathrm{M}$ final concentration) and samples were incubated in the dark at room temperature for 2 hours. $100 \mu \mathrm{L}$ of glacial acetic acid was added to acidify samples and the mBB-CoA derivative was purified using a 2-(2-pyridyl) ethyl-functionalized silica gel column (Supelco). Samples were dried 
using nitrogen flow and were stored at $-20^{\circ} \mathrm{C}$ prior to analysis. Samples were analyzed via high pressure liquid chromatography as described previously (27).

To determine the effect of Nudt8 over-expression on the (acyl-)CoA pool composition, CoA species were analyzed using a protocol adapted from Minkler P.E. et al. (28). Samples were analyzed via LC/MS/MS at the Metabolome Analysis Facility at West Virginia University on an $A B$ Sciex QTrap 5500 mass spectrometer connected to an AB Sciex Exion UPLC as described previously (16).

\section{Immunoblotting}

For western blot analysis, flash frozen tissues were homogenized in radioimmunoprecipitation assay buffer containing protease inhibitors and were centrifuged at $10,000 \mathrm{xg}$ for 10 minutes at $4^{\circ} \mathrm{C}$. Sample were fractionated by SDS/PAGE on $4-12 \%$ Bis-Tris gels and transferred to PVDF membrane. The Nudt8 antibody, was used at 1:3000 dilution. The alpha tubulin antibody was used at a 1:2000 dilution. Primary antibodies were detected using HRP-conjugated goat anti-rabbit lgG a 1:22,000 dilution.

\section{Results}

\section{Submitochondrial Localization of Nudt8}

Using immunofluorescence and subcellular fractionation, Nudt8 was shown to localize to the mitochondria (18). Further, proximity tagging experiments have shown that human NUDT8 localizes to the matrix of the mitochondria (29). To confirm this for the mouse isoform, we utilized a protease protection assay in which freshly isolated mitochondria were treated with proteinase $\mathrm{K}$ and increasing concentrations of digitonin. Digitonin in a nonionic detergent that binds to cholesterol disrupting the outer mitochondrial membrane (OMM) in a concentration dependent manner. At low concentrations of digitonin, the OMM will remain intact and the protease will be unable to access the inner membrane/matrix. Treatment with intermediate concentration of 
digitonin (0.05\%-0.06\%) will result in partial disruption of the OMM, and proteins localizing to the inner membrane (IM) and inner membrane space (IMS) will start to be degraded. At high concentrations of digitonin, the OMM will be completely solubilized and proteins localizing to the IM and IMS will be completely degraded. Proteins localizing to the matrix will be protected from protease degradation throughout digitonin treatments except for treatment with Triton-X-100 which completely solubilizes the mitochondria allowing for the protease to access all mitochondrial compartments. Tim23, a marker of the inner membrane, was degraded at intermediate concentrations of digitonin, as expected (Fig. 3.1). While citrate synthase, which resides in the mitochondrial matrix, was only digested in the Triton-X-100, which completely lyses the mitochondria. Nudt8 was protected from degradation in all the digitonin conditions and was only degraded with the addition of Triton-X-100 (Figure 3.1). Overall, the degradation pattern of Nudt8 was identical to that of citrate synthase indicating that it localizes to the mitochondrial matrix.

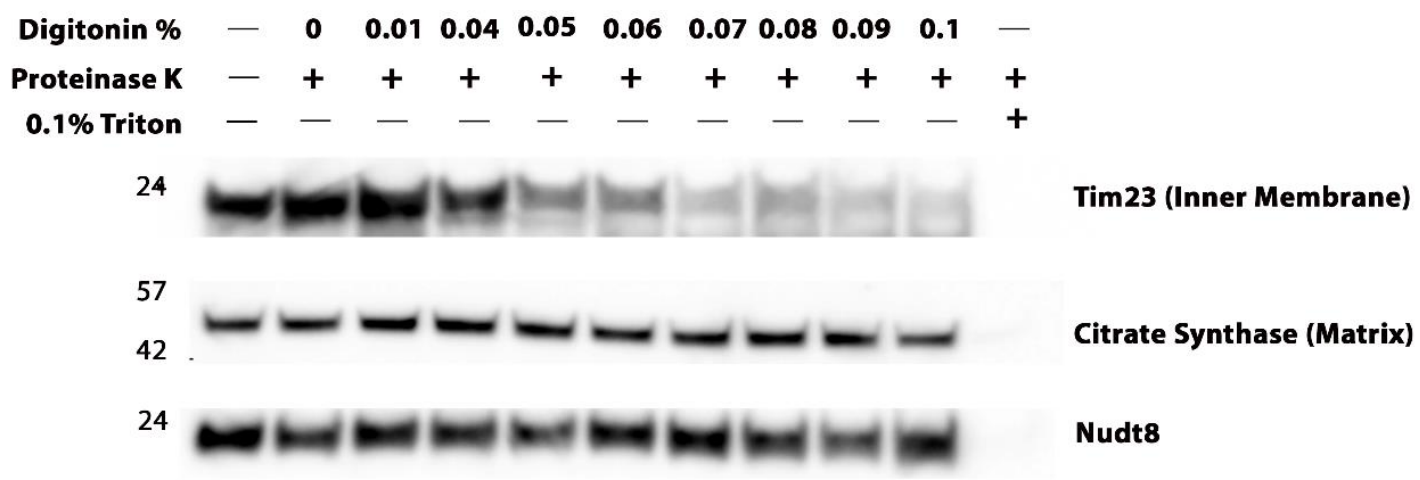

Figure 3.1. Nudt8 localizes to the mitochondrial matrix. Protease protection experiments were utilized to determine the submitochondrial localization of Nud8t using proteinase $\mathrm{K}$ and increasing concentrations of digitonin. Mitochondria treated with $0.3 \%$ Triton served as positive control as it completely solubilizes both the outer and inner mitochondrial membranes. Tim23 was used to mark the mitochondrial inner membrane. Citrate synthase was used to mark the mitochondrial matrix. Representative of three independent experiments.

To confirm these findings by an independent assay, we utilized proximity tagging using a ascorbate peroxidase (APEX) localizing to the mitochondrial matrix (29). APEX is an engineered peroxidase that utilizes hydrogen peroxide to react with biotinylated-phenol. This reaction creates 
a hydroxy radical that covalently reacts with nearby proteins (approximately $20 \mathrm{~nm}$ in proximity) (29). Expression of the APEX within the mitochondrial matrix will result in labeling of the matrix proteome. Thus, we expected that proteins localizing to the mitochondrial matrix such as Citrate synthase and Nudt8 would be biotinylated as a result of the APEX expression. Conversely, proteins that are outside of the mitochondrial matrix, such as cytochrome $\mathrm{C}$ should not be biotinylated. The plasmid encoding APEX was transiently transfected into NIH-3T3 cells stably expressing Nudt8 with a C-terminal FLAG tag. Biotinylation of Nudt8 was assessed via immunoprecipitation using the FLAG antibody. Citrate synthase, known to localize to the matrix, was used as a positive control, and cytochrome $\mathrm{C}$, which localizes to the inter membrane space, was used as a negative control. Immunoprecipitated proteins were fractionated via SDS-PAGE and analyzed via western blot. To determine if proteins were biotinylated, membranes were probed with streptavidin (Fig. 3.2, A and B). As anticipated, biotinylation only occurred with the expression of the APEX, addition of hydrogen peroxide and biotinylated phenol (Fig. 3.2, A and B, lane 4). Immunoprecipitated Nudt8 and citrate synthase were detected using the streptavidinHRP conjugate but were not seen in the negative control lacking $\mathrm{H}_{2} \mathrm{O}_{2}$ (Fig. 3.2, C-E). However, probing elution fractions with antibodies for Nudt8 and citrate synthase, respectively, showed that the immunoprecipitation was successful (Fig. 3.2, D and F). The Nudt8 immunoprecipitation was performed with an antibody recognizing the FLAG epitope and immunoblotted for Nudt8 using an antibody that has previously been shown to be specific for the mouse isoform of Nudt8 (18). Immunoprecipitated cytochrome C, a marker of the intermembrane space was not biotinylated indicating that biotinylation is specific to the mitochondrial matrix (Fig. 3.2, G-H). Importantly, using an APEX construct targeted to the mitochondrial inter membrane space, cytochrome $\mathrm{C}$ was biotinylated (30). This supports our conclusion that biotininylation by this matrix targeted APEX construct is specific to the mitochondrial matrix. Taken together, this confirms the localization of Nudt8 to the mitochondrial matrix. 
A

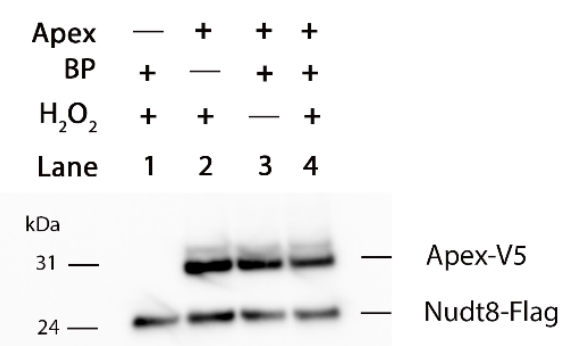

B

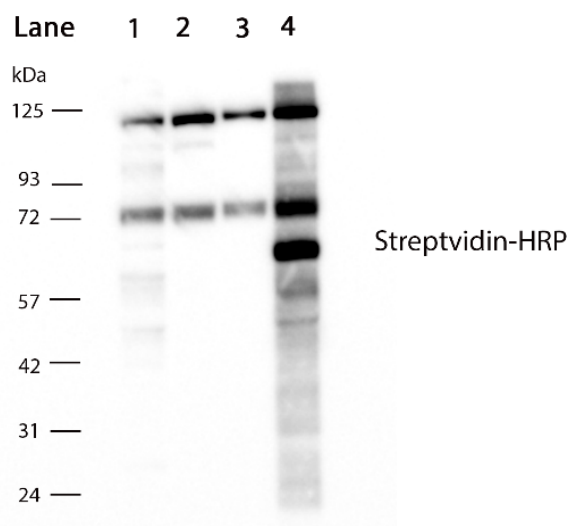

C

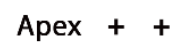

$\mathrm{BP}++$

$\mathrm{H}_{2} \mathrm{O}_{2}-+$

$57--\quad$ IP:CS
IB: Strep

$42-$

E

Apex ++

$\mathrm{BP}++$

$\mathrm{H}_{2} \mathrm{O}_{2}-+$

31 -

IP:Flag
IB: Strep

G

Apex + +

$\mathrm{BP}++$

$\mathrm{H}_{2} \mathrm{O}_{2}-+$

17 -

$15-$
D
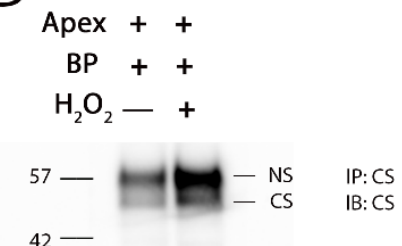

$42-$

F

Apex ++

$\mathrm{BP}++$

$\mathrm{H}_{2} \mathrm{O}_{2}-+$

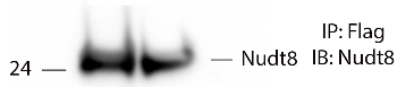

$\mathrm{H}$

Apex + +

$\mathrm{BP}++$

$\mathrm{H}_{2} \mathrm{O}_{2}-+$

$17-$

Figure 3.2. APEX mediated proximity biotinylation localizes Nudt8 to the mitochondrial matrix. A) Transient expression of APEX with a V5 tag in NIH-3T3 cells stably expressing Nudt8FLAG. Nudt8 was identified using antibody recognizing the FLAG epitope. APEX expression was identified using an antibody against the V5 epitope. B) Biotinylation in cellular lysates of NIH-3T3 cells. Lane 1 (mock transfection), lane 2 (no Biotinylated phenol, BP) and Lane 3 (no $\mathrm{H}_{2} \mathrm{O}_{2}$ ) served as negative controls. Lane 4 showed increased biotinylation as a result of the APEX expression and treatment with $\mathrm{BP}$ and $\mathrm{H}_{2} \mathrm{O}_{2}$. C-D) Citrate synthase (CS) was immunoprecipitated from cell lysates. Bioyinylated CS was identified using Strep-HRP conjugated antibody (C). Panel D shows immunoprecipitated CS as identified by the CS antibody. E-F) (E) shows biotinylation in lane 2 as identified by Streptavidin-HRP antibody. (F) Immunoprecipitated Nudt8 was identified using Nudt8 antibody. ( $\mathrm{G}-\mathrm{H})$ Cytochrome $\mathrm{C}$ was used as a negative control as it resides in the intermembrane space. Immunoprecipitated Cyt $C$ was probed with Streptavidin-HRP antibody to identify the biotinylation $(\mathrm{G})$. Panel $\mathrm{H}$ shows immunoprecipitated Cyt $\mathrm{C}$ as identified by $\mathrm{Cyt} \mathrm{C}$ antibody.

\section{Liver Specific Expression of Nudt8 Transgene}

Mice injected with Nudt8-AAV experienced a robust expression of the transgene when compared to non-injected mice (Fig. 3.3, A). Previously, we showed that the ApoE/AAT1 promoter results in liver specific expression of the transgene (16). As Nudt8 is a mitochondrial protein, we 
wanted to ensure that the transgene was properly localized. To address this, we isolated primary hepatocytes from mice injected with Nudt8-AAV and utilized immunofluorescence and confocal microscopy to determine the subcellular localization of the transgene. The transgene was identified using antibody recognizing the FLAG epitope on the C-terminus of the protein. Mitochondria were labeled with Mitotracker, and $\mathbf{A}$ nuclei with DAPI. FLAGtagged Nudt8 appeared to have a morphology and pattern that mirrored that

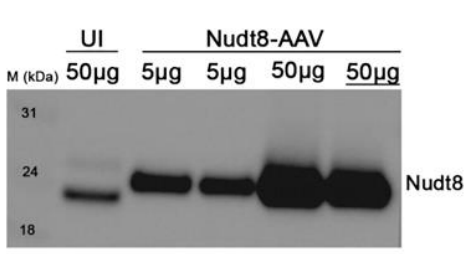
of the Mitotracker, and the
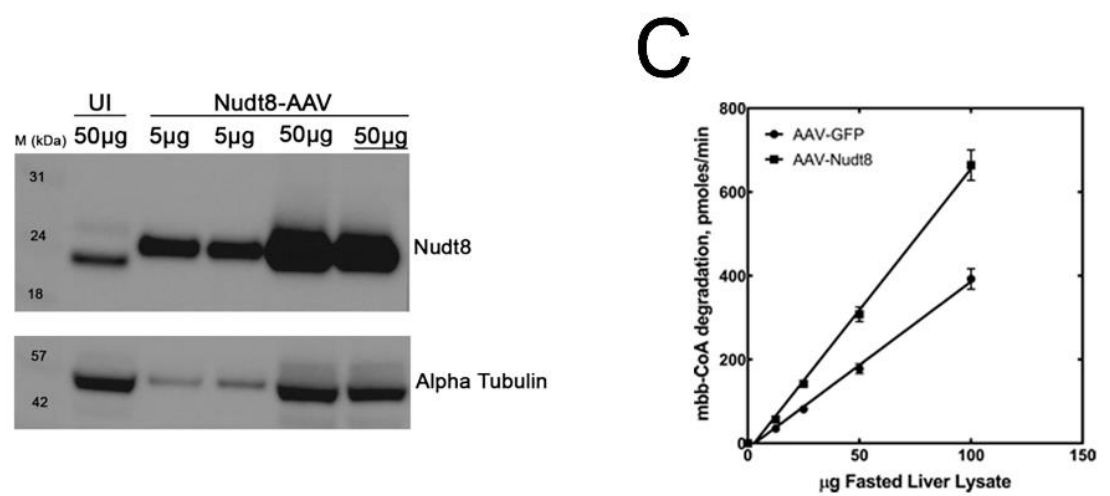

overlay the transgene largely colocalized with the Mitotracker (Fig, 3.3 B). We cannot, however, exclude that a minor portion of the Nudt8-FLAG localized to other
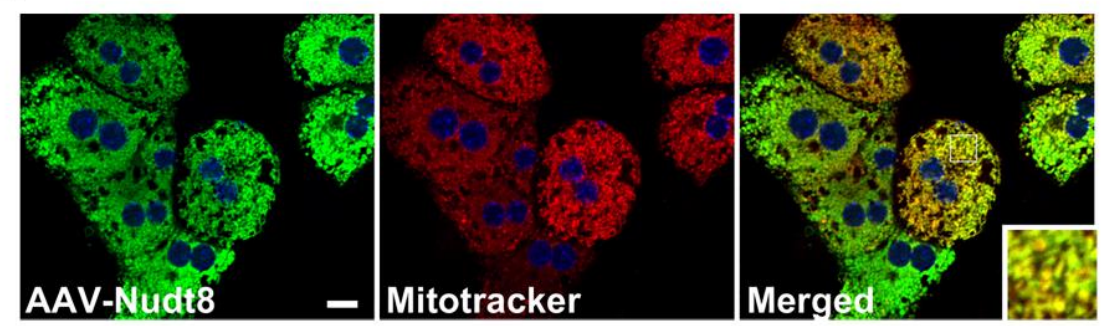

subcellular compartments.

This could be determined by

immunofluorescence using additional markers for other

Figure 3.3. Liver specific over-expression of Nudt8 increased the total CoA-degrading activity in the mitochondria. C57BL/6J mice were injected with AAV particles to achieve liver-specific overexpression of Nudt8-Flag or the GFP control. A) Western blot analysis of liver lysates 3weeks following injection of Nudt8-Flag or GFP transgene. B) Primary hepatocytes were isolated from Nudt8-AAV mice and fixed. The FLAG antibody was used to visualize the Nudt8 transgene. Mitochondria were visualized using mitotracker. Cell nuclei were stained with DAPI. The inset in the merged panel shows higher magnification. C) Liver lysates of Nudt8AAV and GFP injected mice were assayed for total CoA-degrading activity. Experiments were performed in triplicate. Data are reported as mean \pm SEM.

organelles or by subcellular fractionation techniques. Additionally, background fluorescence from the FLAG antibody could be determined using a non-injected mouse. Next, we examined the effect of Nudt8 overexpression on total CoA diphosphohydrolase activity of Nudt8-AAV and GFP. AAV liver lysates. To minimize the potential background resulting from Nudt7-mediated CoA 
degradation, the dominant CoA-degrading isoform in the liver, we measured CoA-degrading activity in tissue harvested following 24-hour fast when Nudt7 expression is lowest (16). The Nudt8- AAV mice were associated with a 1.5- fold increase in CoA phosphohydrolase activity compared to GFP controls (Fig. 3.3 C). Taken together, these results show robust expression of the transgene in the mitochondria, which resulted in increased CoA-degrading activity liver lysates.

\section{Effect of Nudt8 on Blood Glucose and $\beta$-Hydroxybutyrate}

In the fasted state, mitochondrial fatty acid $\beta$-oxidation relies on an increase in free CoA generated from CoA biosynthesis (10). Acetyl-CoA generated from fatty acid $\beta$-oxidation contributes to increased flux through the gluconeogenic pathway through allosteric activation of pyruvate carboxylase (3). Likewise, acetoacetyl-CoA for synthesis of $\beta$-hydroxybutyrate is generated from acetyl-CoA generated

from mitochondrial fatty acid oxidation

(3). Inability to synthesize adequate

amounts of free $\mathrm{CoA}$, in the fasted state, reduces fatty acid oxidation resulting in impaired gluconeogenesis and ketone synthesis (10). We hypothesized that overexpression Nudt8 in the mitochondrial matrix

A

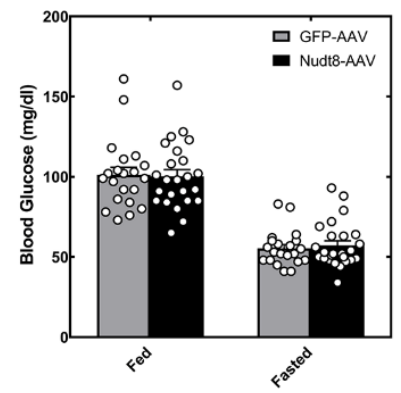

B

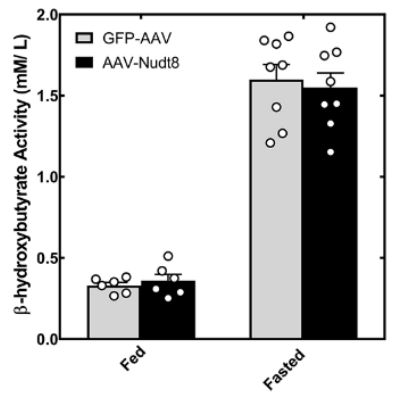

Fig 3.4. Blood glucose and $\beta$-hydroxybutyrate were not affected by Nudt8 over-expression. A) Serum glucose in the AAV-GFP and AAV Nudt8 in the fed and 24 hour fasted state. B) $\beta$-hydroxybutyrate in serum of fed and 24 hour fasted AAV-GFP and AAV- Nudt8 mice.

would reduce the availability of free CoA, which in vitro is an excellent substrate for Nudt8 (18). This reduction in free CoA would reduce the acetyl-CoA generated from mitochondrial fatty acid oxidation resulting in decreased flux through the gluconeogenic pathway leading to fasting hypoglycemia and decreased serum $\beta$-hydroxybutyrate. Nudt8 overexpression had no effect on blood glucose or $\beta$-hydroxybutyrate in either the fed or the fasted state (Fig.3.4). This suggests 
that the increase in CoA degradation due to Nudt8 overexpression likely did not decrease the free CoA enough to effect mitochondrial fatty acid oxidation.
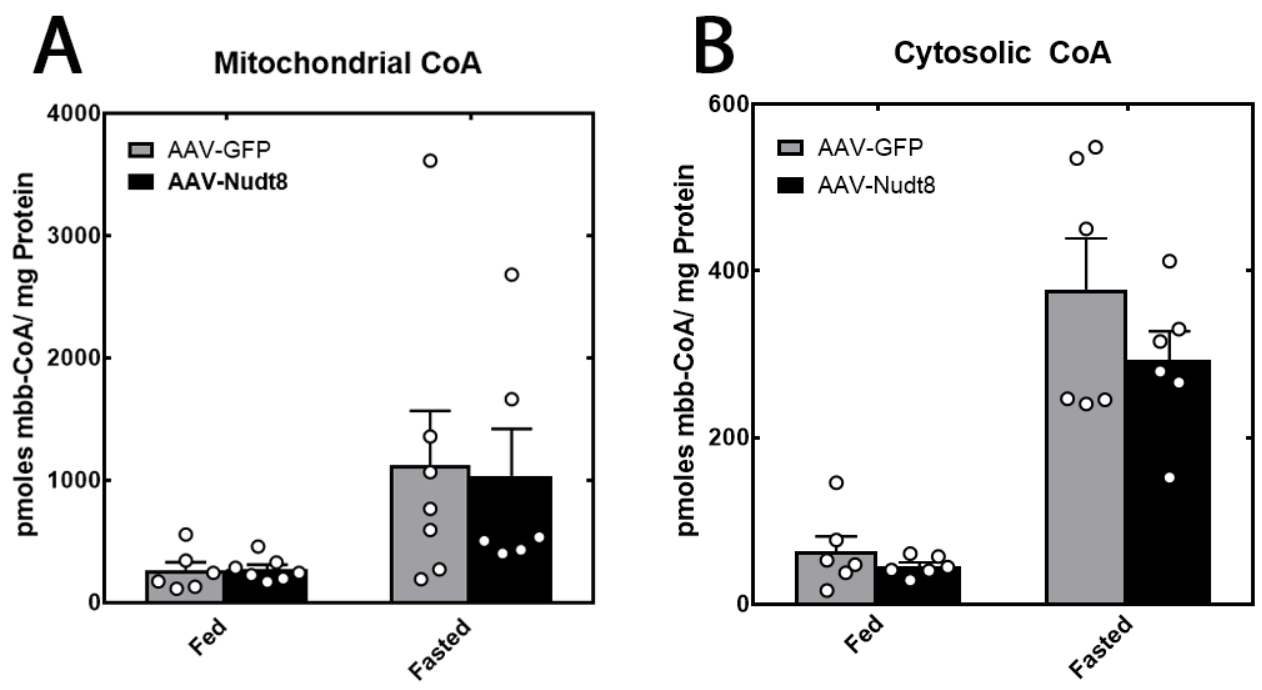

Figure 3.5. Mitochondrial and cytosolic total CoA did not change as a result of Nudt8 over-expression. Liver mitochondria and cytosolic fractions were isolated from fed and 24-h fasted Nudt8-AAV and GFP injected mice. Total CoA was measured in each fraction from ad libitum fed and 24 hour fasted mice. Data are reported as mean \pm SEM.

\section{Effect of Nudt8 on the Size and Composition of the CoA Pool}

The pool of CoA in the mitochondrial matrix is isolated from the rest of the cell due to the fact that the mitochondrial inner membrane is impermeable to CoA species. We hypothesized that overexpression of Nudt8, which would increase CoA-degradation within the mitochondrial matrix, would decrease total CoA in the mitochondria when compared to the GFP controls. Mitochondrial and cytosolic fractions from fed and 24 hour fasted Nudt8-AAV and GFP control mice were obtained by subcellular fractionation. Total CoA was assessed via derivatization with mBB and quantification by HPLC. There was no significant difference in the magnitude of the total CoA pool in either the mitochondria or cytosolic fractions in the fasted or the fed state (Figure 3.5). 
Next, we sought to determine if Nudt8 overexpression had an impact on the composition of the acyl-CoA pool in liver lysates. In vitro analysis of recombinantly expressed an purified Nudt8 showed it was a promiscuous CoA diphosphohydrolase that readily hydrolyzes free CoA as well as malonyl-, succinyl-, hexanoyl- and octanoyl-CoA (18). However, longer chain acyl-CoAs could not be assayed because of precipitation under our enzymatic assay conditions. To determine in vivo substrates of Nudt8, we analyzed the changes in the composition of the acyl-CoA pool that occurred as a result of over-expression of the Nudt8 using LC/MS/MS. In the fasted state, there were no differences in free CoA or acetyl-CoA (Fig. 3.6A). Likewise, there were no differences in
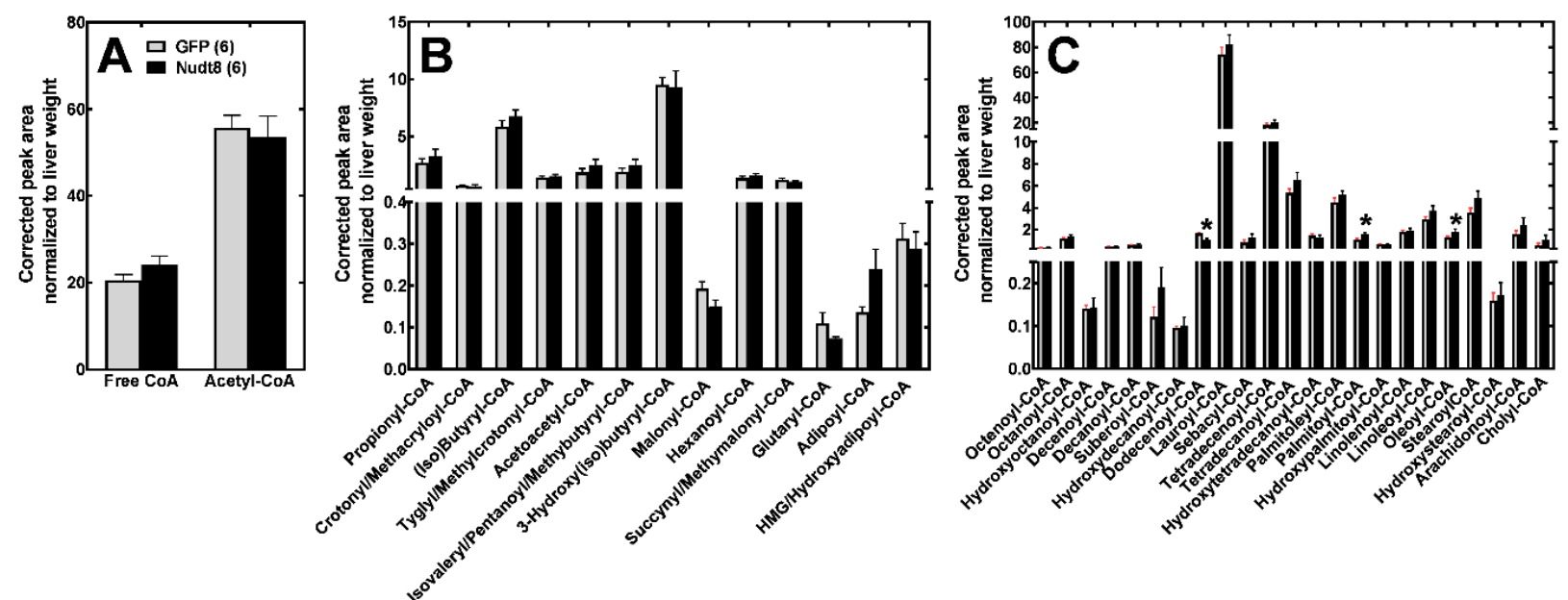

Figure 3.6. Acyl-CoA analysis from 24 hour fasted liver. Acyl-CoA composition was analyzed in 24 hour fasted Nudt8-AAV and GFP mice via LC/MS/MS. Relative abundance of (A) free and acetyl-CoA, B) Shortchain acyl-CoAs and C) long-chain acyl-CoAs was determined from a total of 6 mice per group. * Indicates $p<0.05$. Data is reported as mean \pm SEM.

any of the short-chain acyl-CoAs identified by mass spectrometry (Fig. 3.6 B). Examining the effect of Nudt8 overexpression on long-chain acyl-CoAs, there was a significant decrease in dodecenoyl-CoA. Additionally, there were small but significant increases in palmitoyl-CoA and oleoyl-CoA (Fig 3.6 C). Taken together, these results show that overexpression of Nudt8 had no significant effect on total CoA levels, with minor changes to the composition of the acyl-CoA pool. 


\section{Discussion}

Recently, Nudt8 was shown to be a novel CoA diphosphohydrolase that localized to the mitochondria (18). Here we report that Nudt8 localizes to the mitochondrial matrix, positioning it to regulate the largest (acyl-) CoA pool in the cell (9). To gain insight into its role in CoA regulation, we overexpressed Nudt8 in mouse liver using an AAV. Given the localization of Nudt8 to the mitochondrial matrix, we anticipated that over-expression of Nudt8 would decrease the amount of one or more CoA species, including free CoA. This decrease in free $\mathrm{CoA}$, in turn, would reduce acetyl-CoA available for ketogenesis and would also reduce flux through the gluconeogenic pathway resulting in fasting hypoglycemia. Expression of the transgene resulted in a 1.5- fold increase in CoA-degrading activity in liver lysates compared to GFP-AAV controls; however, this modest increases in CoA-degrading activity had no effect on total CoA and only minor changes to the composition of the acyl-CoA pool (Fig 3.5 and 3.6). The lack of changes, despite an increase in CoA degradation, can be potentially attributed to compensation by increase in CoA biosynthesis.

The Nudt8 transgene was correctly localized to the mitochondria. In the liver, mitochondria have been estimated to contain as much as $80 \%$ of the total cellular CoA, with the remaining $20 \%$ distributed amongst the cytosol and the peroxisomes (16,31-33). Increases in CoA degradation in the peroxisomes through over-expression of the peroxisomal Nudt7 was restricted to the peroxisomes (16). Thus, we hypothesized that overexpression of mitochondrial Nudt8 would act to regulate the much larger mitochondrial (acyl-)CoA pool. While expression of the Nudt8 transgene did increase the total CoA-degrading activity detected in the liver of mice injected with Nudt8-AAV, the magnitude of the total mitochondrial (acyl-)CoA pool was not affected (Fig 3.5). One potential explanation for the lack of phenotype is compensation by the CoA biosynthetic pathway. Decreases in mitochondrial CoA due to increased Nudt8 activity could trigger import of CoA from the cytosol, through active transport into the mitochondrial matrix. Presently two CoA 
transporters belonging to the SLC family of proteins transport CoA, however, the direction of the transport (i.e. import into the matrix or export from the matrix) has yet to be determined $(19,21)$. The resulting shift in cytosolic CoA into the mitochondria could, in turn, trigger increase activity of Pantothenate Kinase (PanK), which would increase CoA biosynthesis returning cytosolic CoA to normal levels.

The CoA-degrading activity of Nudt8 could also have been mitigated through the activity of CoA synthase (Coasy) (Fig 3.7). Coasy catalyzes the final two steps in CoA biosynthesis and has previously been shown to localize to the outer mitochondrial membrane (34). However, recently, Coasy has been shown to localize to the mitochondrial matrix $(29,35)$. If this finding is confirmed it places both CoA biosynthetic and CoA-degrading proteins within the same compartment, raising the question as to how each is regulated to prevent futile cycles of synthesis and degradation. Nudt8 is not regulated transcriptionally (18) and is thus potentially regulated via posttranslational modification or allosterically, so that activity is minimized in the fasted state and maximized in the fed state. While Coasy, on the other hand, would be active in the fasted state to promote CoA biosynthesis. Despite recent findings localizing Coasy to the mitochondrial matrix, previous research showed interactions with ribosomal S6 kinase, enhancer of mRNA-decapping protein 4 , and the p85 subunit of PI3 kinase, which contradict the matrix localization as all of these enzymes are cytosolic (36-40). Thus, determining the subcellular localization of Coasy will be integral for the understanding of where the cellular CoA pool is synthesized and how it relates to CoA degradation occurring in the mitochondria. 


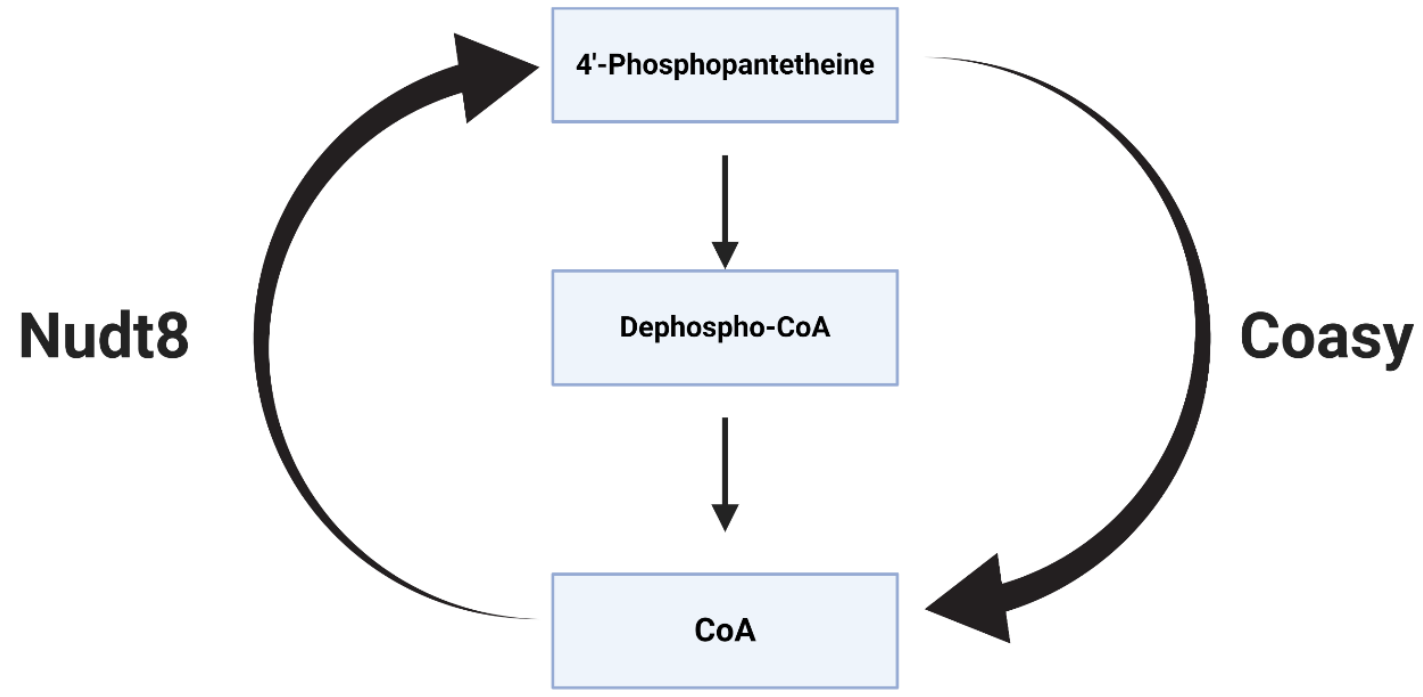

Figure 3.7. Compensation of Nudt8 mediated CoA degradation by Coasy. Increase in CoA degradation due to overexpression of Nudt8 could have been compensated for by the presence of Coasy. Nudt8 hydrolyzes CoA in to 3'5'-ADP and 4'- phosphopantetheine which, in turn could be substrate for Coasy localized to the mitochondrial matrix. 


\section{References:}

1. Brass, E. P. (1994) Overview of coenzyme A metabolism and its role in cellular toxicity. Chem Biol Interact 90, 203-214

2. Leonardi, R., Zhang, Y. M., Rock, C. O., and Jackowski, S. (2005) Coenzyme A: back in action. Prog Lipid Res 44, 125-153

3. Pietrocola, F., Galluzzi, L., Bravo-San Pedro, J. M., Madeo, F., and Kroemer, G. (2015) Acetyl coenzyme A: a central metabolite and second messenger. Cell Metab 21, 805-821

4. Trefely, S., Lovell, C. D., Snyder, N. W., and Wellen, K. E. (2020) Compartmentalised acyl-CoA metabolism and roles in chromatin regulation. Mol Metab 38, 100941

5. Hirschey, M. D., Shimazu, T., Goetzman, E., Jing, E., Schwer, B., Lombard, D. B., Grueter, C. A., Harris, C., Biddinger, S., Ilkayeva, O. R., Stevens, R. D., Li, Y., Saha, A. K., Ruderman, N. B., Bain, J. R., Newgard, C. B., Farese, R. V., Jr., Alt, F. W., Kahn, C. R., and Verdin, E. (2010) SIRT3 regulates mitochondrial fatty-acid oxidation by reversible enzyme deacetylation. Nature 464, 121-125

6. Park, J., Chen, Y., Tishkoff, D. X., Peng, C., Tan, M., Dai, L., Xie, Z., Zhang, Y., Zwaans, B. M. M., Skinner, M. E., Lombard, D. B., and Zhao, Y. (2013) SIRT5-mediated lysine desuccinylation impacts diverse metabolic pathways. Mol Cell 50, 919-930

7. Gout, I. (2018) Coenzyme A, protein CoAlation and redox regulation in mammalian cells. Biochem Soc Trans 46, 721-728

8. Gout, I. (2018) Coenzyme A, protein CoAlation and redox regulation in mammalian cells. Biochemical Society Transactions 46, 721-728

9. Naquet, P., Kerr, E. W., Vickers, S. D., and Leonardi, R. (2020) Regulation of coenzyme A levels by degradation: the 'Ins and Outs'. Prog Lipid Res 78, 101028

10. Leonardi, R., Rehg, J. E., Rock, C. O., and Jackowski, S. (2010) Pantothenate kinase 1 is required to support the metabolic transition from the fed to the fasted state. PLoS One 5, e11107

11. Leonardi, R., Rock, C. O., and Jackowski, S. (2014) Pank1 deletion in leptin-deficient mice reduces hyperglycaemia and hyperinsulinaemia and modifies global metabolism without affecting insulin resistance. Diabetologia 57, 1466-1475

12. Zhang, Y. M., Chohnan, S., Virga, K. G., Stevens, R. D., Ilkayeva, O. R., Wenner, B. R., Bain, J. R., Newgard, C. B., Lee, R. E., Rock, C. O., and Jackowski, S. (2007) Chemical knockout of pantothenate kinase reveals the metabolic and genetic program responsible for hepatic coenzyme A homeostasis. Chem Biol 14, 291-302

13. Corbin, D. R., Rehg, J. E., Shepherd, D. L., Stoilov, P., Percifield, R. J., Horner, L., Frase, S., Zhang, Y. M., Rock, C. O., Hollander, J. M., Jackowski, S., and Leonardi, R. (2017) Excess coenzyme A reduces skeletal muscle performance and strength in mice overexpressing human PANK2. Mol Genet Metab 120, 350-362

14. Gasmi, L., and McLennan, A. G. (2001) The mouse Nudt7 gene encodes a peroxisomal nudix hydrolase specific for coenzyme A and its derivatives. The Biochemical journal 357, 33-38

15. Ofman, R., Speijer, D., Leen, R., and Wanders, R. J. A. (2006) Proteomic analysis of mouse kidney peroxisomes: identification of RP2p as a peroxisomal nudix hydrolase with acyl-CoA diphosphatase activity. The Biochemical journal 393, 537-543

16. Shumar, S. A., Kerr, E. W., Fagone, P., Infante, A. M., and Leonardi, R. (2019) Overexpression of Nudt7 decreases bile acid levels and peroxisomal fatty acid oxidation in the liver. J Lipid Res 60, 1005-1019

17. Shumar, S. A., Kerr, E. W., Geldenhuys, W. J., Montgomery, G. E., Fagone, P., Thirawatananond, P., Saavedra, H., Gabelli, S. B., and Leonardi, R. (2018) Nudt19 is a 
renal CoA diphosphohydrolase with biochemical and regulatory properties that are distinct from the hepatic Nudt7 isoform. J Biol Chem 293, 4134-4148

18. Kerr, E. W., Shumar, S. A., and Leonardi, R. (2019) Nudt8 is a novel CoA diphosphohydrolase that resides in the mitochondria. FEBS Lett 593, 1133-1143

19. Fiermonte, G., Paradies, E., Todisco, S., Marobbio, C. M., and Palmieri, F. (2009) A novel member of solute carrier family 25 (SLC25A42) is a transporter of coenzyme A and adenosine 3',5'-diphosphate in human mitochondria. J Biol Chem 284, 18152-18159

20. Kim, Y. I., Nam, I. K., Lee, D. K., Bhandari, S., Charton, L., Kwak, S., Lim, J. Y., Hong, K., Kim, S. J., Lee, J. N., Kwon, S. W., So, H. S., Linka, N., Park, R., and Choe, S. K. (2020) Slc25a17 acts as a peroxisomal coenzyme $A$ transporter and regulates multiorgan development in zebrafish. J Cell Physiol 235, 151-165

21. Prohl, C., Pelzer, W., Diekert, K., Kmita, H., Bedekovics, T., Kispal, G., and Lill, R. (2001) The yeast mitochondrial carrier Leu5p and its human homologue Graves' disease protein are required for accumulation of coenzyme A in the matrix. Mol Cell Biol 21, 1089-1097

22. Shumar, S. A., Kerr, E. W., Geldenhuys, W. J., Montgomery, G. E., Fagone, P., Thirawatananond, P., Saavedra, H., Gabelli, S. B., and Leonardi, R. (2018) Nudt19 is a renal CoA diphosphohydrolase with biochemical and regulatory properties that are distinct from the hepatic Nudt7 isoform. The Journal of biological chemistry

23. Cyphert, H. A., Alonge, K. M., Ippagunta, S. M., and Hillgartner, F. B. (2014) Glucagon stimulates hepatic FGF21 secretion through a PKA- and EPAC-dependent posttranscriptional mechanism. PLoS One 9, e94996

24. Graham, J. M. (2001) Isolation of mitochondria from tissues and cells by differential centrifugation. Curr Protoc Cell Biol Chapter 3, Unit 33

25. Bekeova, C., Anderson-Pullinger, L., Boye, K., Boos, F., Sharpadskaya, Y., Herrmann, J. M., and Seifert, E. L. (2019) Multiple mitochondrial thioesterases have distinct tissue and substrate specificity and CoA regulation, suggesting unique functional roles. The Journal of biological chemistry 294, 19034-19047

26. Hung, V., Udeshi, N. D., Lam, S. S., Loh, K. H., Cox, K. J., Pedram, K., Carr, S. A., and Ting, A. Y. (2016) Spatially resolved proteomic mapping in living cells with the engineered peroxidase APEX2. Nature protocols 11, 456-475

27. Shumar, S. A., Fagone, P., Alfonso-Pecchio, A., Gray, J. T., Rehg, J. E., Jackowski, S., and Leonardi, R. (2015) Induction of Neuron-Specific Degradation of Coenzyme A Models Pantothenate Kinase-Associated Neurodegeneration by Reducing Motor Coordination in Mice. PLoS One 10, e0130013

28. Minkler, P. E., Kerner, J., Ingalls, S. T., and Hoppel, C. L. (2008) Novel isolation procedure for short-, medium-, and long-chain acyl-coenzyme A esters from tissue. Anal Biochem 376, 275-276

29. Rhee, H. W., Zou, P., Udeshi, N. D., Martell, J. D., Mootha, V. K., Carr, S. A., and Ting, A. Y. (2013) Proteomic mapping of mitochondria in living cells via spatially restricted enzymatic tagging. Science (New York, N.Y.) 339, 1328-1331

30. Hung, V., Zou, P., Rhee, H.-W., Udeshi, Namrata D., Cracan, V., Svinkina, T., Carr, Steven A., Mootha, Vamsi K., and Ting, Alice Y. (2014) Proteomic Mapping of the Human Mitochondrial Intermembrane Space in Live Cells via Ratiometric APEX Tagging. Mol Cell 55, 332-341

31. Horie, S., Ishii, H., and Suga, T. (1981) Changes in peroxisomal fatty acid oxidation in the diabetic rat liver. J Biochem 90, 1691-1696

32. Smith, C. M. (1978) The effect of metabolic state on incorportion of [14C] pantothenate into CoA in rat liver and heart. $J$ Nutr 108, 863-873

33. Smith, C. M., Cano, M. L., and Potyraj, J. (1978) The relationship between metabolic state and total CoA content of rat liver and heart. $J$ Nutr 108, 854-862 
34. Zhyvoloup, A., Nemazanyy, I., Panasyuk, G., Valovka, T., Fenton, T., Rebholz, H., Wang, M. L., Foxon, R., Lyzogubov, V., Usenko, V., Kyyamova, R., Gorbenko, O., Matsuka, G., Filonenko, V., and Gout, I. T. (2003) Subcellular localization and regulation of coenzyme A synthase. J Biol Chem 278, 50316-50321

35. Dusi, S., Valletta, L., Haack, T. B., Tsuchiya, Y., Venco, P., Pasqualato, S., Goffrini, P., Tigano, M., Demchenko, N., Wieland, T., Schwarzmayr, T., Strom, T. M., Invernizzi, F., Garavaglia, B., Gregory, A., Sanford, L., Hamada, J., Bettencourt, C., Houlden, H., Chiapparini, L., Zorzi, G., Kurian, M. A., Nardocci, N., Prokisch, H., Hayflick, S., Gout, I., and Tiranti, V. (2014) Exome sequence reveals mutations in CoA synthase as a cause of neurodegeneration with brain iron accumulation. American journal of human genetics 94, 11-22

36. Breus, O., Panasyuk, G., Gout, I. T., Filonenko, V., and Nemazanyy, I. (2010) CoA Synthase is phosphorylated on tyrosines in mammalian cells, interacts with and is dephosphorylated by Shp2PTP. Molecular and Cellular Biochemistry 335, 195-202

37. Nemazanyy, I., Panasyuk, G., Zhyvoloup, A., Panayotou, G., Gout, I. T., and Filonenko, V. (2004) Specific interaction between S6K1 and CoA synthase: a potential link between the mTOR/S6K pathway, CoA biosynthesis and energy metabolism. FEBS Letters 578, 357-362

38. Arif, A., Jia, J., Willard, B., Li, X., and Fox, P. L. (2019) Multisite Phosphorylation of S6K1 Directs a Kinase Phospho-code that Determines Substrate Selection. Mol Cell 73, 446457.e446

39. Gudkova, D., Panasyuk, G., Nemazanyy, I., Zhyvoloup, A., Monteil, P., Filonenko, V., and Gout, I. (2012) EDC4 interacts with and regulates the dephospho-CoA kinase activity of CoA synthase. FEBS Lett 586, 3590-3595

40. Breus, O., Panasyuk, G., Gout, I. T., Filonenko, V., and Nemazanyy, I. (2009) CoA Synthase is in complex with p85aPI3K and affects PI3K signaling pathway. Biochemical and Biophysical Research Communications 385, 581-585 


\section{Chapter IV: Conclusions and Future Directions}

CoA is an essential cofactor that functions as an acyl-donor and participates in hundreds of metabolic reactions (1). Beyond its direct participation in metabolic reactions, (acyl-)CoAs, regulate metabolism through allosteric interactions, and, through post-translational modification, can regulate protein activity and gene expression $(2,3)$. In this way, changes in the levels of acylCoAs as well as the composition of the (acyl-)CoA pool, can act to 'fine tune' gene expression and protein activity to match the energy needs of the cell. Metabolic processes activated during fasting, like fatty acid $\beta$-oxidation, ketogenesis, and gluconeogenesis are driven by an increase in free CoA which is achieved through increased CoA synthesis (4). Conversely, the transition from fatty oxidation to lipogenesis and glucose oxidation is driven by decreased synthesis and active degradation of free $\mathrm{CoA}$ and thioesters. Indeed, simply decreasing the rate of CoA synthesis would not achieve a net decrease in the concentration of CoA species accumulated during the fast without CoA/acyl-CoA degradation. Previously, the decrease in total CoA during the fasted-to- fed transition, in the liver and kidneys, was attributed to the peroxisomal CoAdegrading enzymes Nudt7 and Nudt19 respectively (5). However, deletion of Nudt19 does not completely prevent the decrease in total CoA levels that characterize the transition from the fasted to fed state, indicating the presence of other CoA-degrading enzymes (6). Furthermore, while liver-specific overexpression of Nudt7 in the liver affected peroxisomal lipid metabolism, it had no detectible effect on total hepatic CoA levels (7). These findings indicate that Nudt7 and Nudt19 likely function to regulate the small peroxisomal (acyl-)CoA pool. However, they also support the existence of additional CoA-degrading enzymes that regulate the larger mitochondria and cytosolic (acyl-)CoA pools. 
Nudt8 has been annotated as a CoA-degrading enzyme due to its sequence similarity to Nudt7. Additionally, Nudt8 was predicted to localize to the mitochondria, the organelles containing the largest (acyl-)CoA pool in the cell $(8,9)$. The objective of this thesis was to 1) test ability the ability of Nudt8 to degrade CoA species using recombinantly expressed and purified protein, 2) determine its tissue distribution and subcellular distribution and 3) establish, in vivo, the physiological role of Nudt8 regarding (acyl-)CoA regulation.

\section{Conclusions from Chapter II:}

To establish Nudt8 as a CoA diphosphohydrolase, we utilized in vitro enzymatic assays with recombinantly expressed and purified protein. The main finding that emerged from this approach is that Nudt8 is a CoA-specific diphosphohydrolase that hydrolyzes (acyl-)CoA in to 3',5'-ADP and (acyl-)phosphopantotheine (10). We found that recombinant Nudt8 was capable of degrading free CoA as well as a variety of short-, medium- and dicarboxylic acyl-CoAs (10).

Additionally, Nudt8 activity was dependent on the presence of $\mathrm{Mn}^{2+}$. The Nudix motif, common to all Nudix hydrolase enzymes, binds divalent cation $\left(\mathrm{Mn}^{2+}\right.$ or $\left.\mathrm{Mg}^{2+}\right)$ which, in turn, binds and activates a water molecule that ultimately hydrolyzes phosphodiester bonds of the substrate molecule $(11,12)$. Nudt8 and Nudt7 have a Nudix box that are largely identical, thus the finding that Nudt8 activity is $\mathrm{Mn}^{2+}$ dependent was unexpected. Finally, we were able to show that Nudt8 is a mitochondrial enzyme and has a broad tissue distribution, as it is expressed the liver, kidneys, heart, brown adipose tissue and skeletal muscle (10). The mitochondrial localization of Nudt8 positions it to regulate the size and composition of the largest pool of (acyl-)CoA pool in the cell.

\section{Future Directions of Chapter II:}

Mitochondria consist of four distinct compartments: the outer membrane, the intermembrane space (IMS), the inner-membrane, and the matrix. The outer membrane consists of pores that enable (acyl-)CoAs to freely diffuse into the IMS from the cytosol, thus the (acyl- 
)CoA pool in the IMS and in the cytosol are thought to be at equilibrium (13). The inner membrane of the mitochondria is impermeable to (acyl-)CoAs, and the matrix pool of free CoA is likely derived via counter-exchange transporters belonging to the Solute Carrier 25 (SLC25) family of proteins (14-16). Due to the existence of two separate pools of (acyl-)CoAs within the mitochondria, it was essential to determine the submitochondrial localization of Nudt8. Localization of Nudt8 to the IMS would indicate that its activity would function to regulate the IMS/ cytosolic pool of (acyl)CoAs. Conversely, localization of Nudt8 to the mitochondrial matrix would indicate it regulates the larger pool in the matrix, potentially modulating processes like fatty acid oxidation, ketogenesis, and gluconeogenesis.

Prior to this work, Nudt8 had been annotated as a CoA-degrading enzyme due to its similarity to Nudt7. Despite the similarities of Nudt8 and Nudt7 in the CoA binding region and the Nudix box, we found several important differences between these CoA diphosphohydrolases. The first difference was the potent activation of Nudt8 by $\mathrm{Mn}^{2+}$. In vitro, Nudt8 activity was completely

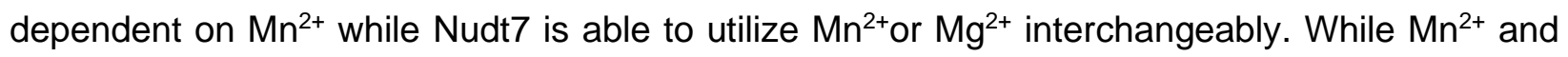
$\mathrm{Mg}^{2+}$ are usually considered surrogates for each other, $\mathrm{Mn}^{2+}$ forms ligands with nitrogen and sulfur containing amino acids (eg. histidine, cysteines) and forms bidentate complexes with oxygencontaining ligands, like glutamate, more readily than $\mathrm{Mg}^{2+}(17,18)$. The ligands formed with $\mathrm{Mn}^{2+}$ are more flexible in both length and angle than $\mathrm{Mg}^{2+}$, which adheres to a strict octahedral ligand binding geometry (18). A second important difference that we saw between Nudt7 and Nudt8 were differences in substrate preferences. While both enzymes are promiscuous CoA-degrading enzymes, Nudt8 showed the lowest activity towards acetyl-CoA and short-chain acyl-CoAs, which are preferred substrates of Nudt7 $(7,19,20)$. This raises the question as to whether Nudt8s requirement of $\mathrm{Mn}^{2+}$ plays a role in the substrate specificity of the enzyme. The ability of $\mathrm{Mn}^{2+}$ to form more flexible ligand bonds with amino acids in the Nudix box of Nudt8 may impart a unique conformation that promotes the binding of dicarboxylic and medium chain acyl-CoAs, at the 
expense of shorter chain acyl-CoAs. To confirm this, the crystal structures of Nudt8 and Nudt7 bound to substrate and divalent cations will be necessary to show exactly how each metal effects substrate binding and activity of each respective protein.

In the fasted state, CoA levels increase due to increased biosynthesis and decreased degradation (9). Upon refeeding, there is an increase in (acyl-)CoA degradation that results in a rapid decrease in tissue (acyl-)CoA levels (7). We hypothesized that Nudt8 expression would change with the nutritional state, with minimal expression in the fasted state and increased expression in the fed state. Nudt8 mRNA and protein levels did not change as a result of the nutritional state in liver, kidney or heart (10). A potential alternative is that Nudt8 activity is potentially regulated through posttranslational modification (PTM) or allosteric interactions. PTMs such as acetylation and succinylation are emerging as important regulators of mitochondrial metabolism, thus Nudt8 activity could potentially be dependent on deacetylases such Sirt3 and Sirt5 (21-24). To determine if Nudt8 is regulated via post translational modification or proteinprotein interaction, we can utilize immunoprecipitation coupled with either immunoblotting or mass spectrometry to analyze how Nudt8 is modified and how it changes with nutritional state. Alternately, Nudt8 activity could be regulated allosterically or competitively through via mitochondrial metabolites. Alternately, Nudt8 activity could be regulated allosterically or competitively through via mitochondrial metabolites. Nudt8 also possesses two, predicted, hydrophobic domains, one on the N-terminus (residues 33-51) and the other towards the Cterminus (residues142-197) $(25,26)$. Nudt8 activity and stability could be regulated through interaction with lipids, the mitochondrial inner membrane, or other proteins via hydrophobic interactions. To address this, recombinant Nudt8 activity can be measured in the presence of mitochondrial lipids and metabolites to determine if there is activation or inhibition. 


\section{Conclusions Chapter III:}

We showed, in vitro, that Nudt8 is a specific CoA diphosphohydrolase that localizes to the mitochondria (10). Our next aim was to determine the submitocondrial localization of Nudt8 and to identify metabolic processes effected by Nudt8-mediated CoA degradation. Our main finding was that Nudt8 localizes to the mitochondrial matrix (Fig 3.1 and 3.2). The mitochondrial matrix contains the largest concentration of free and acyl-CoA thioesters in the cell, and we hypothesized that Nudt8 would reduce the $\mathrm{CoA}$ and acyl-CoA thioesters within the mitochondrial matrix to transition from the fasted to the fed state.

To elucidate in vivo substrates of Nudt8, we utilized a liver specific adeno-associated virus to overexpress Nudt8 in the liver of $\mathrm{C} 57 \mathrm{BI} / 6 \mathrm{~J}$ mice. We were able to robustly overexpress the Nudt8 transgene in the liver and this expression resulted in increased (acyl-)CoA-degrading activity in liver lysates when compared to GFP injected controls (Fig. 3.3). Despite the increase in CoA degradation, Nudt8 overexpression had no effect on the total CoA levels in the mitochondria or cytosolic fractions (Fig. 3.5). Next, we analyzed changes in individual changes in acyl-CoAs via mass spectrometry and found no significant alterations to the hepatic CoA as a result of Nudt8 overexpression (Fig. 3.6).

\section{Future Directions Chapter III:}

Overexpression of Nudt8 in the liver had no measurable effect on mitochondrial total CoA levels and only minor changes in the acyl-CoA composition of the liver. One possible explanation is that the increased degradation of CoA that occurred due to the transgene expression was compensated for by increased biosynthesis. The decrease in mitochondrial CoA due to Nudt8 overexpression could have resulted in increased importation of cytosolic CoA into the mitochondria. The resulting decrease in cytosolic CoA would be compensated for through increased activity of PanK, the rate limiting step in CoA biosynthesis (1). To address this 
hypothesis, we could compare the flux through the CoA biosynthetic pathway using radiolabeled pantothenate in primary hepatocytes isolated from Nudt8-AAV and GFP-AAV injected mice respectively. We would expect accumulation of radiolabeled CoA in the Nudt8-AAV indicating increased CoA biosynthesis.

The presence of CoA synthase (Coasy) in the mitochondria presents an alternate means through which CoA biosynthesis could compensate for increased Nudt8 mediated CoAdegradation. Coasy is a bifunctional enzyme that catalyzes the formation of dephospho-CoA from 4'-phosphopantotheine as well as phosphorylation of dephospho-CoA to CoA, the final step in CoA biosynthesis. Initially, Coasy was thought to localize to the outer membrane of the mitochondria, however, more recently it was found to localize to the matrix $(27,28)$. If Coasy does in fact localize to the mitochondrial matrix, then 4'-phosphopantotheine generated from Nudt8 could be reincorporated into CoA by Coasy, mitigating the activity of Nudt8 (Fig. 3.7). This raises the question as to how biosynthesis (Coasy) and degradation (Nudt8), within the mitochondria, are regulated to prevent futile cycles of synthesis and degradation. Coasy has been shown to interact with multiple proteins effecting its activity including enhancer of mRNA- decapping 4 (EDC4), p85, and S6K1 (29-33). The effect of these interactions on CoA biosynthesis have not been elucidated in vivo. However, interaction with EDC4 and phosphorylation via S6K1 are likely inhibitory $(29,33)$. Interestingly, these interacting proteins are cytosolic, and thus it presently unclear how they can act to regulate Coasy activity within the mitochondria. One potential explanation is the localization of Coasy in the mitochondrial matrix as well as the cytosol. To address this hypothesis, we could utilize simple subcellular fractionation techniques to isolate mitochondrial and cytosolic fractions and look for Coasy expression in each respective fraction. Next, we can immunoprecipitated Coasy from mitochondrial and cytosolic fractions from fasted and fed tissues and examine differences in post translational modifications and protein-protein 
interactions. This will provide insight into 1) if two separate pools of Coasy exist and 2) identify an alternate mechanism through which CoA biosynthesis is regulated.

\section{Conclusion:}

Nudt8 is a novel CoA diphosphohydrolase that localizes to the mitochondrial matrix. The discovery of Nudt8 has identified a potentially important mechanism for regulating mitochondrial (acyl-)CoA levels and thus mitochondrial metabolism. The mitochondria contain between $80 \%$ $95 \%$ of the total cellular CoA supporting flux through the TCA cycle, fatty acid $\beta$-oxidation, ketogenesis and gluconeogenesis (9). The fast to fed transition in the liver, kidney and heart is associated with a dramatic decrease in free $\mathrm{CoA}$, which is abundant in the mitochondrial matrix $(9,34)$. In vitro, free CoA is readily hydrolyzed by Nudt8, thus it could function to reduce the availability of free CoA for fatty acid oxidation within the mitochondrial matrix (10). Additionally, Nudt8 could function to regulate long-chain acyl-CoAs, however, we were not able to test this activity in vitro due to substrate precipitation.

Additionally, the finding that Nudt8 and Coasy localize to the mitochondrial matrix raises the question of how CoA degradation and biosynthesis are regulated within the mitochondria, as well as where CoA is ultimately synthesized. Previously, it was thought that Coasy localized to the outer mitochondrial membrane, thus its activity would result in the accumulation of newly synthesized CoA in the cytosol (35). However, the recent finding Coasy localizes to the mitochondrial matrix challenges this paradigm $(27,28)$. As phosphopantetheine is negatively charged and exhibits very limited ability to passively diffuse across membranes, it would require a transporter that, to this point, has not been identified (36). Furthermore, if the final two steps of CoA biosynthesis occur in the mitochondria, the rate of CoA biosynthesis would be dependent on the rate at which phosphopantheine can be imported into the mitochondrial matrix and the rate at which newly synthesized CoA can be exported. Since PANK enzymes are well known to act as sensors of CoA levels in the cytosol, nucleus and mitochondrial IMS, which can equilibrate 
metabolites within the cytosol, a scenario in which CoA is synthesized in the mitochondrial matrix is hard to reconcile with this evidence. Due to the fact that Coasy interacts with multiple proteins known to localize outside the mitochondria, it is likely that Coasy localizes to both the mitochondria and the cytosol. The cytosolic Coasy could function as the primary site of CoA biosynthesis, while the mitochondrial Coasy could function to regenerate CoA from the phosphopantetheine produced by Nudt8.

Alternatively, as no PANK isoform localizes to the mitochondrial matrix, Nudt8 and Coasy could act cooperatively, acting as local sensors for free CoA and could modify the acyl-CoA/ free CoA ratio. In conditions where free CoA is limiting, 3',5'-ADP generated from Nudt8- mediated degradation of acyl-CoAs activity could stimulate the import of free CoA into the mitochondrial matrix via the SLC25A42 and SLC25A16 transport proteins. The acyl-phosphopantetheine generated by Nudt8, could be hydrolyzed by an acyl-CoA thioesterase and the resulting phosphopantetheine then could serve as substrate for Coasy. This would essentially result in the generation of two free $\mathrm{CoA}$ molecules and alter the acyl-CoA/ CoA ratio within the mitochondria. This could prove advantageous in situations when free CoA is limiting. Future studies, including the generation of a Nudt8 ${ }^{-/}$mouse are needed to elucidate how Nudt8 regulates the mitochondrial CoA pool and thus mitochondrial metabolism.

In conclusion, we have identified Nudt8 as a novel CoA diphosphohydrolase that localizes to the mitochondrial matrix. Further, we have shown that CoA degradation occurs outside of the peroxisomes and in a broad array of tissues including the liver, kidney, heart, brown adipose tissue and skeletal muscle. Overall, this discovery has identified a novel mechanism through which the mitochondrial acyl-CoAs are regulated. 


\section{$\underline{\text { References }}$}

1. Leonardi, R., Zhang, Y. M., Rock, C. O., and Jackowski, S. (2005) Coenzyme A: back in action. Prog Lipid Res 44, 125-153

2. Pietrocola, F., Galluzzi, L., Bravo-San Pedro, J. M., Madeo, F., and Kroemer, G. (2015) Acetyl coenzyme A: a central metabolite and second messenger. Cell Metab 21, 805-821

3. Trefely, S., Lovell, C. D., Snyder, N. W., and Wellen, K. E. (2020) Compartmentalised acyl-CoA metabolism and roles in chromatin regulation. Mol Metab 38, 100941

4. Leonardi, R., Rehg, J. E., Rock, C. O., and Jackowski, S. (2010) Pantothenate kinase 1 is required to support the metabolic transition from the fed to the fasted state. PLoS One 5, e11107

5. Zhang, Y. M., Chohnan, S., Virga, K. G., Stevens, R. D., Ilkayeva, O. R., Wenner, B. R., Bain, J. R., Newgard, C. B., Lee, R. E., Rock, C. O., and Jackowski, S. (2007) Chemical knockout of pantothenate kinase reveals the metabolic and genetic program responsible for hepatic coenzyme A homeostasis. Chem Biol 14, 291-302

6. Shumar, S. A., Kerr, E. W., Geldenhuys, W. J., Montgomery, G. E., Fagone, P., Thirawatananond, P., Saavedra, H., Gabelli, S. B., and Leonardi, R. (2018) Nudt19 is a renal CoA diphosphohydrolase with biochemical and regulatory properties that are distinct from the hepatic Nudt7 isoform. J Biol Chem 293, 4134-4148

7. Shumar, S. A., Kerr, E. W., Fagone, P., Infante, A. M., and Leonardi, R. (2019) Overexpression of Nudt7 decreases bile acid levels and peroxisomal fatty acid oxidation in the liver. J Lipid Res 60, 1005-1019

8. Calvo, S. E., Clauser, K. R., and Mootha, V. K. (2016) MitoCarta2.0: an updated inventory of mammalian mitochondrial proteins. Nucleic Acids Res 44, D1251-1257

9. Naquet, P., Kerr, E. W., Vickers, S. D., and Leonardi, R. (2020) Regulation of coenzyme A levels by degradation: the 'Ins and Outs'. Prog Lipid Res 78, 101028

10. Kerr, E. W., Shumar, S. A., and Leonardi, R. (2019) Nudt8 is a novel CoA diphosphohydrolase that resides in the mitochondria. FEBS Lett 593, 1133-1143

11. McLennan, A. G. (2006) The Nudix hydrolase superfamily. Cellular and molecular life sciences : CMLS 63, 123-143

12. Mildvan, A. S., Xia, Z., Azurmendi, H. F., Saraswat, V., Legler, P. M., Massiah, M. A., Gabelli, S. B., Bianchet, M. A., Kang, L. W., and Amzel, L. M. (2005) Structures and mechanisms of Nudix hydrolases. Archives of Biochemistry and Biophysics 433, 129-143

13. Lemasters, J. J. (2007) Modulation of mitochondrial membrane permeability in pathogenesis, autophagy and control of metabolism. Journal of Gastroenterology and Hepatology 22, S31-S37

14. Iuso, A., Alhaddad, B., Weigel, C., Kotzaeridou, U., Mastantuono, E., Schwarzmayr, T., Graf, E., Terrile, C., Prokisch, H., Strom, T. M., Hoffmann, G. F., Meitinger, T., and Haack, T. B. (2019) A Homozygous Splice Site Mutation in SLC25A42, Encoding the Mitochondrial Transporter of Coenzyme A, Causes Metabolic Crises and Epileptic Encephalopathy. JIMD Rep 44, 1-7

15. Palmieri, F. (2013) The mitochondrial transporter family SLC25: identification, properties and physiopathology. Mol Aspects Med 34, 465-484

16. Prohl, C., Pelzer, W., Diekert, K., Kmita, H., Bedekovics, T., Kispal, G., and Lill, R. (2001) The yeast mitochondrial carrier Leu5p and its human homologue Graves' disease protein are required for accumulation of coenzyme A in the matrix. Mol Cell Biol 21, 1089-1097

17. Bock, C. W., Katz, A. K., Markham, G. D., and Glusker, J. P. (1999) Manganese as a Replacement for Magnesium and Zinc: Functional Comparison of the Divalent lons. Journal of the American Chemical Society 121, 7360-7372 
18. Kehres, D. G., and Maguire, M. E. (2003) Emerging themes in manganese transport, biochemistry and pathogenesis in bacteria. FEMS Microbiology Reviews 27, 263-290

19. Gasmi, L., and McLennan, A. G. (2001) The mouse Nudt7 gene encodes a peroxisomal nudix hydrolase specific for coenzyme A and its derivatives. The Biochemical journal 357 , 33-38

20. Reilly, S.-J., Tillander, V., Ofman, R., Alexson, S. E. H., and Hunt, M. C. (2008) The Nudix Hydrolase 7 is an Acyl-CoA Diphosphatase Involved in Regulating Peroxisomal Coenzyme A Homeostasis. The Journal of Biochemistry 144, 655-663

21. Choudhary, C., Weinert, B. T., Nishida, Y., Verdin, E., and Mann, M. (2014) The growing landscape of lysine acetylation links metabolism and cell signalling. Nature Reviews Molecular Cell Biology 15, 536-550

22. Hirschey, M. D., Shimazu, T., Goetzman, E., Jing, E., Schwer, B., Lombard, D. B., Grueter, C. A., Harris, C., Biddinger, S., Ilkayeva, O. R., Stevens, R. D., Li, Y., Saha, A. K., Ruderman, N. B., Bain, J. R., Newgard, C. B., Farese, R. V., Jr., Alt, F. W., Kahn, C. R., and Verdin, E. (2010) SIRT3 regulates mitochondrial fatty-acid oxidation by reversible enzyme deacetylation. Nature 464, 121-125

23. Park, J., Chen, Y., Tishkoff, D. X., Peng, C., Tan, M., Dai, L., Xie, Z., Zhang, Y., Zwaans, B. M. M., Skinner, M. E., Lombard, D. B., and Zhao, Y. (2013) SIRT5-mediated lysine desuccinylation impacts diverse metabolic pathways. Mol Cell 50, 919-930

24. Zhang, Y., Bharathi, S. S., Rardin, M. J., Lu, J., Maringer, K. V., Sims-Lucas, S., Prochownik, E. V., Gibson, B. W., and Goetzman, E. S. (2017) Lysine desuccinylase SIRT5 binds to cardiolipin and regulates the electron transport chain. J Biol Chem 292, 10239-10249

25. Kyte, J., and Doolittle, R. F. (1982) A simple method for displaying the hydropathic character of a protein. Journal of Molecular Biology 157, 105-132

26. Snider, C., Jayasinghe, S., Hristova, K., and White, S. H. (2009) MPEx: a tool for exploring membrane proteins. Protein Sci 18, 2624-2628

27. Dusi, S., Valletta, L., Haack, T. B., Tsuchiya, Y., Venco, P., Pasqualato, S., Goffrini, P., Tigano, M., Demchenko, N., Wieland, T., Schwarzmayr, T., Strom, T. M., Invernizzi, F., Garavaglia, B., Gregory, A., Sanford, L., Hamada, J., Bettencourt, C., Houlden, H., Chiapparini, L., Zorzi, G., Kurian, M. A., Nardocci, N., Prokisch, H., Hayflick, S., Gout, I., and Tiranti, V. (2014) Exome sequence reveals mutations in CoA synthase as a cause of neurodegeneration with brain iron accumulation. American journal of human genetics 94 , 11-22

28. Rhee, H. W., Zou, P., Udeshi, N. D., Martell, J. D., Mootha, V. K., Carr, S. A., and Ting, A. Y. (2013) Proteomic mapping of mitochondria in living cells via spatially restricted enzymatic tagging. Science (New York, N.Y.) 339, 1328-1331

29. Gudkova, D., Panasyuk, G., Nemazanyy, I., Zhyvoloup, A., Monteil, P., Filonenko, V., and Gout, I. (2012) EDC4 interacts with and regulates the dephospho-CoA kinase activity of CoA synthase. FEBS Lett 586, 3590-3595

30. Breus, O., Panasyuk, G., Gout, I. T., Filonenko, V., and Nemazanyy, I. (2009) CoA Synthase is in complex with p85aPI3K and affects PI3K signaling pathway. Biochemical and Biophysical Research Communications 385, 581-585

31. Ferrandon, S., DeVecchio, J., Duraes, L., Chouhan, H., Karagkounis, G., Davenport, J., Orloff, M., Liska, D., and Kalady, M. F. (2020) CoA Synthase (COASY) Mediates Radiation Resistance via PI3K Signaling in Rectal Cancer. Cancer Res 80, 334-346

32. Arif, A., Jia, J., Willard, B., Li, X., and Fox, P. L. (2019) Multisite Phosphorylation of S6K1 Directs a Kinase Phospho-code that Determines Substrate Selection. Mol Cell 73, 446457.e446

33. Nemazanyy, I., Panasyuk, G., Zhyvoloup, A., Panayotou, G., Gout, I. T., and Filonenko, V. (2004) Specific interaction between S6K1 and CoA synthase: a potential link between 
the mTOR/S6K pathway, CoA biosynthesis and energy metabolism. FEBS Letters 578, 357-362

34. Tokutake, Y., Onizawa, N., Katoh, H., Toyoda, A., and Chohnan, S. (2010) Coenzyme A and its thioester pools in fasted and fed rat tissues. Biochemical and Biophysical Research Communications 402, 158-162

35. Zhyvoloup, A., Nemazanyy, I., Panasyuk, G., Valovka, T., Fenton, T., Rebholz, H., Wang, M. L., Foxon, R., Lyzogubov, V., Usenko, V., Kyyamova, R., Gorbenko, O., Matsuka, G., Filonenko, V., and Gout, I. T. (2003) Subcellular localization and regulation of coenzyme A synthase. J Biol Chem 278, 50316-50321

36. Srinivasan, B., Baratashvili, M., van der Zwaag, M., Kanon, B., Colombelli, C., Lambrechts, R. A., Schaap, O., Nollen, E. A., Podgoršek, A., Kosec, G., Petković, H., Hayflick, S., Tiranti, V., Reijngoud, D.-J., Grzeschik, N. A., and Sibon, O. C. M. (2015) Extracellular 4'-phosphopantetheine is a source for intracellular coenzyme A synthesis. Nat Chem Biol 11, 784-792 